\title{
Conferencing in Wyner's Asymmetric Interference Network: Effect of Number of Rounds
}

\author{
Michèle Wigger, Senior Member, IEEE, Roy Timo, Member, IEEE, and Shlomo Shamai (Shitz), Fellow, IEEE
}

\begin{abstract}
We determine the exact per-user multiplexing gain (MG) of Wyner's soft-handoff network model when neighbouring transmitters and neighbouring receivers can communicate over rate-limited pipes. The per-user MG is determined, in function of the capacities of these conferencing links and of the maximum number of allowed conferencing rounds. The results reveal a certain duality between transmitter and receiver conferencing. The per-user MG remains unchanged when one exchanges the transmitter-conferencing parameters (conferencing prelog and the maximum number of permitted rounds) and the receiver-conferencing parameters. The results also show that for small conferencing prelogs $(\leq 1 / 6)$, single conferencing rounds suffice to attain the maximum per-user MG when the number of conferencing rounds is unlimited. For moderate conferencingprelogs, multiple (but finite) number of conferencing rounds are required to achieve the same per-user MG as with unlimited conferencing rounds. Finally, for large conferencing prelogs, the per-user MG saturates at a value $<1$ depending on the number of allowed conferencing rounds, and every additional transmitter- or receiver-conferencing round increases the saturation level. With unlimited conferencing rounds the per-user MG is 1 when the transmitter- and receiver-conferencing-prelogs are $\geq 1 / 4$.
\end{abstract}

Index Terms - Cellular networks, user cooperation, degrees of freedom, dirty-paper coding, successive interference cancellation.

\section{INTRODUCTION}

C ONSIDER a future wireless communications network where neighbouring transmitters and neighbouring receivers cooperate over dedicated conferencing links that do not interfere with the main communications channel. Our goal in this work is to determine exactly how the network's performance depends on the number of interactive conferencing rounds that are allowed to take place between the transmitters and receivers. Our motivation for studying this problem rests

Manuscript received January 13, 2016; revised August 17, 2016; accepted October 2, 2016. Date of publication November 29, 2016; date of current version January 18, 2017. M. Wigger was supported by the city of Paris under the Program Emergences. R. Timo was supported by the Alexander von Humboldt Foundation. S. Shamai was supported by the European Union's Horizon 2020 Research and Innovation Programme, under Grant 694630. This paper was presented at the 2015 IEEE Information Theory Workshop.

M. Wigger is with the Communication and Electronics Department, Télécom ParisTech, 75013 Paris, France (e-mail: michele.wigger@telecomparistech.fr).

R. Timo was with the Institute for Communications Engineering the Technical University of Munich, 80333 Munich, Germany. He is now with Ericsson Research, 16483 Stockholm, Sweden (e-mail: roy.timo@ericsson.com).

S. Shamai is with the Department of Electrical Engineering, Technion-Israel Institute of Technology, Haifa 3200003, Israel (e-mail: sshlomo@ee.technion.ac.il).

Communicated by O. Simeone, Associate Editor for Communications.

Color versions of one or more of the figures in this paper are available online at http://ieeexplore.ieee.org.

Digital Object Identifier 10.1109/TIT.2016.2633253 on the fact that conferencing will play an important role in meeting future wireless demands [1], and protocols with fewer conferencing rounds are more desirable in practice. For example, conferencing is often subject to stringent latency constraints, and the transmitters and receivers often have limited memory and computational capabilities.

Willems' [2] showed that a single conferencing round (during which the transmitters exchange parts of their messages) is optimal for the two-user discrete memoryless multi-access channel (MAC). The same conclusion holds for the two-user memoryless Gaussian MAC [3], [4]; the three-user Gaussian or discrete memoryless MAC, where the transmitters cooperate over "public links" that are observed by all transmitters [5]; and for the compound discrete memoryless MAC [6]. For the three-user memoryless Gaussian MAC with "private" conferencing links where each transmitter can send cooperation information only to its left-neighbour, two conferencing rounds (during which the transmitters share and relay parts of their messages) are sum-rate optimal at high signal-to-noise ratio (SNR) [7].

A single conferencing round is also optimal for the physically degraded two-user discrete memoryless broadcast channel (BC) with conferencing receivers [8]. For general broadcast channels, however, this does not seem to be the case, and Dabora and Servetto proposed an interactive two-round conferencing protocol [8], [9]. Interactive two-round conferencing protocols have also been proposed for two-user discrete memoryless BCs for scenarios where both receivers are interested in the same message or where only one of the two receivers has a message to decode [10]. Conferencing has been studied in many other scenarios (e.g., [10]-[17]), with a general focus on small networks with one or two conferencing rounds.

In this work we consider a large interference network with $K$ (arbitrary large) transmitters and receivers, and we analyze how the network's performance depends on the number of conferencing rounds. Specifically, we consider Wyner's asymmetric soft-handoff model [18]-[20] shown in Figure 1. Here the $K$ transmitters and receivers are aligned on opposite grids, and each transmitted signal is received at the corresponding receiver and the receiver to its right. Although Wyner's model is a significant simplification of real-world cellular systems, it does offer valuable information-theoretic insights for systems that operate with many simultaneously active users [21]. For example, the model appears to be particularly relevant for high-demand reliable high-rate communications in modern cellular systems, where different cells/micro-cell/users 


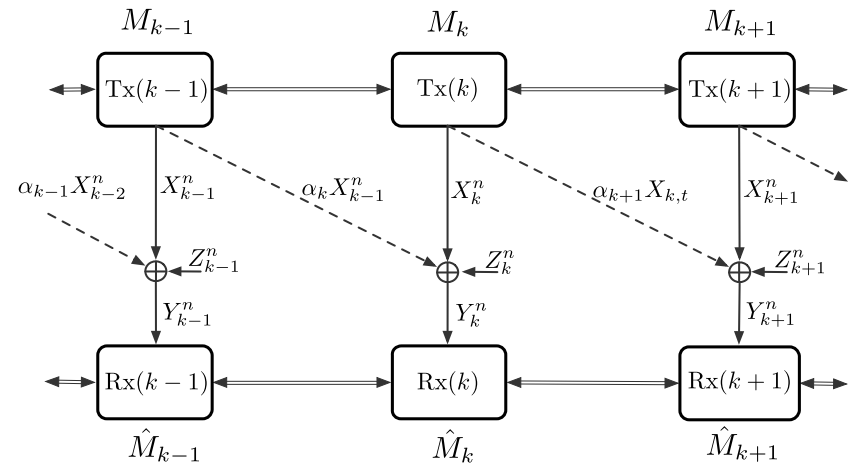

Fig. 1. Wyner's asymmetric interference network with rate-limited noiseless cooperation links between neighbouring transmitters and neighbouring receivers.

simultaneously share the same resources (time/frequency/ space). We have adopted Wyner's model in this work because it provides a tractable framework and ideal starting point for accessing the role of cooperation in such systems.

Each transmitter can conference with its immediate left- and right-neighbours over dedicated non-interfering conferencing links of capacity

$$
\mu_{\mathrm{Tx}} \cdot \frac{1}{2} \log (1+\mathrm{SNR})
$$

where this conferencing communication can take place over at most $\kappa_{\mathrm{Tx}}$ rounds. (SNR stands for signal-to-noise ratio.) Each receiver can conference with its immediate left- and rightneighbours over dedicated non-interfering links of capacity

$$
\mu_{\mathrm{Rx}} \cdot \frac{1}{2} \log (1+\mathrm{SNR}),
$$

over at most $\kappa_{\mathrm{Rx}}$ rounds.

For the Wyner model in Figure 1 this implies that information about a transmitter's message can propagate only as far as its $\kappa_{\mathrm{Tx}}$ left- and right-neighbours, and information about a receiver's output signal can propagate only to its $\kappa_{\mathrm{Rx}}$ left- and right-neighbours. Related scenarios with large interference networks have been studied in [22]-[30]. In contrast to this paper, the cooperation-information in [22], [23], [27], [28], and [30] is fixed a priori, and is not treated as a design parameter of the coding schemes. That is, each transmitter is assumed to be aware of some subset of messages and each receiver is assumed to be aware of some subset of receiver signals.

Our measure of performance will be the high-SNR sumcapacity (specifically, the per-user multiplexing gain $(M G)$ ) of the soft-handoff Wyner network. We will characterize the exact per-user MG later in Theorem 2 as a function of both conferencing prelogs $\mu_{\mathrm{Tx}}$ and $\mu_{\mathrm{Rx}}$ and the number of conferencing rounds $\kappa_{\mathrm{Tx}}$ and $\kappa_{\mathrm{Rx}}$. For comparison, in Theorem 1, we also derive the per-user MG when the number of conferencing rounds is not limited.

First of all, our results exhibit a duality relation between transmitter- and receiver-conferencing: The per-user $\mathrm{MG}$ remains unchanged if one exchanges the conferencingparameters $\left(\kappa_{\mathrm{Tx}}, \mu_{\mathrm{Tx}}\right)$ and $\left(\kappa_{\mathrm{Rx}}, \mu_{\mathrm{Rx}}\right)$. Our results further show that for small conferencing prelogs (in particular for
$\left.\mu_{\mathrm{Tx}}, \mu_{\mathrm{Rx}} \leq 1 / 6\right)$ a single conferencing round at the transmitters and receivers allows one to achieve the same per-user MG as when the number of conferencing rounds is unlimited. For large conferencing prelogs, on the other hand, we will see that the maximum per-user MG increases with every additional conferencing round. In particular, with $\kappa_{\mathrm{Tx}}$ transmit and $\kappa_{\mathrm{Rx}}$ receive conferencing rounds, the per-user MG saturates at

$$
\frac{2 \kappa_{\mathrm{Tx}}+2 \kappa_{\mathrm{Rx}}+1}{2 \kappa_{\mathrm{Tx}}+2 \kappa_{\mathrm{Rx}}+2}
$$

for large values of $\mu_{\mathrm{Tx}}$ and $\mu_{\mathrm{Rx}}$. For moderate conferencing prelogs, a finite number of conferencing rounds $>1$ is required to attain the same performance as with unlimited conferencing. In particular, for equal conferencing prelogs $\mu_{\mathrm{Tx}}=\mu_{\mathrm{Rx}}=$ $\mu \in[1 / 6,1 / 4]$, it suffices to have

$$
\kappa_{\mathrm{Tx}}=\kappa_{\mathrm{Rx}}=\left\lceil\frac{2 \mu}{1-4 \mu}\right\rceil
$$

transmitter- and receiver-conferencing rounds to achieve the same per-user MG as with unlimited conferencing. To the best of our knowledge, these results are the first to quantify the impact of the number of conferencing rounds on the capacity of large interference networks.

In interference networks, both transmitter and receiver cooperation allow one to mitigate interference [6], [16], [17], [22]-[24], [27], [30], [32], [36]. Ntranos et al. [24] recently proposed the following cooperation protocol for interference mitigation in large Gaussian networks where the transmitters share quantised versions of their transmit signals and the receivers share parts of their decoded messages. (This is different from many previous works where transmitters share parts of their messages and receivers share quantised versions of their received signals.) Knowledge about other transmitters' input signals allows the transmitters to mitigate the interference caused by these signals using dirty-paper coding. Similarly, knowledge of decoded messages allows receivers to reconstruct interferences and subtract them from received signals. Two drawbacks of these interference mitigation techniques are that they lead to an inevitable increase in communications delay and they allow interference to propagate across the network. Specifically, each transmitter $k$ has to wait until it has obtained the quantisation information about the transmit signals it wishes to mitigate, before it can construct its own input signal $X_{k}^{n}$ and send quantisation information about it to its other neighbours. In a similar way, each receiver has to wait until it obtains the decoded messages pertaining to some of the transmitters that interfere its output signals, before it can decode its own message and send parts of it to its other neighbours.

The coding scheme presented in this paper is inspired by Ntranos, Maddah-Ali, and Caire's scheme in [24]. However, the constraints on the number of cooperation rounds $\kappa_{\mathrm{Tx}}$ and $\kappa_{\mathrm{Rx}}$ require two major changes:

- Since the transmitters and receivers cannot wait infinitely long before producing their cooperation messages, the protocol can only be applied over subsets of the network. This necessitates to periodically silence transmitters in 
the network, which decomposes the network into smaller subnets.

- The interest is to switch off as few transmitters as possible and thus to make the subnets as large as possible. To this end, a sophisticated combination of the described transmitter and receiver interference-mitigation techniques is required. We also introduce slight variations of these techniques that can exploit the cooperation links from the transmitters to their left neighbours and from the receivers to their left neighbours.

For the case $\kappa_{\mathrm{Tx}}=\kappa_{\mathrm{Rx}}=1$, the performance of our schemes can also be achieved using conferencing protocols where the transmitters share parts of their messages and receivers share quantisation information about their receive signals. The advantage of these protocols is that they do not necessitate codebook knowledge during the conferencing phase and can thus be implemented in oblivious scenarios.

\section{A. Notation}

We denote the integers by $\mathbb{Z}$, the positive integers by $\mathbb{Z}^{+}$, and the real numbers by $\mathbb{R}$. Random variables are identified by uppercase letters, e.g. $W$, their alphabets by matching calligraphic font, e.g. $\mathcal{W}$, and elements of an alphabet by lowercase letters, e.g. $w \in \mathcal{W}$. The Cartesian product of $\mathcal{W}$ and $\mathcal{W}^{\prime}$ is $\mathcal{W} \times \mathcal{W}^{\prime}$, and the $n$-fold Cartesian product of $\mathcal{W}$ is $\mathcal{W}^{n}$. For any $n$-tuple of random variables $\left(W_{1}, \ldots, W_{n}\right)$ we use the shorthand notation $W^{n}:=\left(W_{1}, \ldots, W_{n}\right)$.

Given two $n$-dimensional vectors $a^{n}, b^{n} \in \mathbb{R}^{n}$, let $\left\|a^{n}\right\|$ denote the $\ell^{2}$ norm of $a^{n}$ in Euclidean $n$-space, and let $<a^{n}, b^{n}>$ denote the standard inner product of $a^{n}$ and $b^{n}$. Let further $\angle\left(a^{n}, b^{n}\right)$ denote the angle between the two vectors:

$$
\angle\left(a^{n}, b^{n}\right):=\arccos \frac{<a^{n}, b^{n}>}{\left\|a^{n}\right\|\left\|b^{n}\right\|},
$$

where arccos denotes the arc-cosine function.

\section{B. Organisation of Paper}

The rest of this paper is organised as follows. Section II describes the problem setup. Section III shows at hand of a simple example how the number of conferencing rounds and the choice of conferenced signals influences the per-user MG. Section IV states the main results of the paper. Section V proves the achievability of our Theorem 2; Section VI proves the converses to Theorems 1 and 2 .

\section{Problem Setup}

\section{A. Channel Model and Transmit Power Constraint}

Consider a wireless communications system with $K$ pairs of transmitters (Tx) and receivers $(\mathrm{Rx})$, labeled by $k \in$ $\{1, \ldots, K\}$. Assume that the transmitters and receivers are each equipped with a single antenna, and that all channel inputs and outputs are real valued. We imagine a network with short-range interference, à la [18], [19], [29], [30], so that the signal sent by Tx $k$ is only observed by receivers $k$ and $k+1$. Specifically, the time- $t$ channel output at $\operatorname{Rx} k$ is

$$
Y_{k, t}=X_{k, t}+\alpha_{k} X_{k-1, t}+Z_{k, t},
$$

where $X_{k, t}$ and $X_{k-1, t}$ are the symbols sent by Txs $k$ and $k-1$ at time $t$ respectively; $\left\{Z_{k, t}\right\}$ are independent and identically distributed (i.i.d.) standard Gaussians for all $k$ and $t ; \alpha_{k} \neq 0$ is a fixed real number; and $X_{0, t}=0$ for all $t$. A small segment of this short-range interference model is depicted in Figure 1.

Each and every transmitter $k \in\{1, \ldots, K\}$ is required to reliably communicate a source message $M_{k}$ to its corresponding receiver $k$. The source message $M_{k}$ is uniformly distributed on

$$
\mathcal{M}_{k}:=\left\{1, \ldots,\left\lfloor 2^{n R_{k}}\right\rfloor\right\}
$$

where $n$ denotes the blocklength and $R_{k}$ the rate of transmission of source message $M_{k}$. All source messages are independent of each other and of all channel noises. An average block-power constraint $P>0$ is imposed on the transmitted signals:

$$
\frac{1}{n} \sum_{t=1}^{n} X_{k, t}^{2} \leq P, \quad \text { a.s., } \quad \forall k \in\{1, \ldots, K\} .
$$

\section{B. Overview of Conferencing and Communications Phases}

A key feature of this work is that we include ratelimited local cooperation between neighbouring transmitters and neighbouring receivers over dedicated conferencing links. Specifically, we suppose that the communications process consists of the following four phases.

1) Tx-Conferencing Phase: The source messages $\left(M_{1}, M_{2}, \ldots, M_{K}\right)$ are revealed to their respective transmitters, and each transmitter exchanges conferencing messages with its two direct neighbours over dedicated noiseless channels. Each of these conferencing channels has a maximum rate budget of $n R_{\mathrm{Tx}}$ bits. We let the rate budget $R_{\mathrm{Tx}}$ scale with the transmit power constraint $P$ as

$$
R_{\mathrm{Tx}}:=\mu_{\mathrm{Tx}} \frac{1}{2} \log (1+P),
$$

where $\mu_{\mathrm{Tx}} \in[0, \infty)$ is fixed and called the Tx-prelog conferencing constant.

2) Cooperative Communications Phase: The transmitters communicate their source messages over the memoryless interference channel (1). Tx $k$ 's channel inputs are a function of its source message $M_{k}$ and the conferencing messages it received during the Tx-conferencing phase.

3) Rx-Conferencing Phase: The receivers observe their channel outputs, and they exchange conferencing messages with their immediate neighbours over dedicated noiseless channels. Each of these conferencing channels has a maximum rate

$$
R_{\mathrm{Rx}}:=\mu_{\mathrm{Rx}} \frac{1}{2} \log (1+P) .
$$

Here $\mu_{\mathrm{Rx}} \in[0, \infty)$ is fixed and called the Rx-prelog conferencing constant.

4) Decoding Phase: The receivers decode their desired source messages from the channel outputs and conferencing messages received during the $\mathrm{Rx}$-conferencing phase. 
Remark 1: In the above problem formulation, the $T x$-conferencing phase takes place before the communications phase, and the Rx-conferencing phase takes place before the decoding phase. The conferencing phases, however, should not be considered as strictly separated from the communications and decoding phases. In fact, a transmitter might compute and store its transmit signal already during the Tx-conferencing phase. Similarly, a receiver might compute and store its decoded message already during the Rx-conferencing phase. This allows transmitters (resp. receivers) to exchange parts of their transmit signals (resp. decoded messages) over the conferencing links with their neighbours.

We now describe the four communication phases more formally.

\section{Tx-Conferencing Phase With $\kappa_{T x}$ Rounds}

The Tx-conferencing phases consists of $\kappa_{\mathrm{Tx}}$ rounds: In round $j=1,2, \ldots, \kappa_{\mathrm{Tx}}, \mathrm{Tx} k$ sends a conferencing message $U_{k \rightarrow k^{\prime}}^{(j)}$ to its neighbouring Txs $k-1$ and $k+1$. Here, the round- $j$ conferencing message from Tx $k$ to $\operatorname{Tx} k^{\prime} \in$ $\{k-1, k+1\}$ is a function of message $M_{k}$ as well as all conferencing messages sent to $\mathrm{Tx} k$ in previous rounds $1,2, \ldots, j-1$ :

$$
\begin{array}{r}
U_{k \rightarrow k^{\prime}}^{(j)}:=\phi_{k, k^{\prime}}^{(j)}\left(M_{k}, U_{k-1 \rightarrow k}^{(1)}, U_{k+1 \rightarrow k}^{(1)}, U_{k-1 \rightarrow k}^{(2)},\right. \\
\left.\quad U_{k+1 \rightarrow k}^{(2)}, \ldots, U_{k-1 \rightarrow k}^{(j-1)}, U_{k+1 \rightarrow k}^{(j-1)}\right),
\end{array}
$$

where

$$
\begin{aligned}
\phi_{k, k^{\prime}}^{(j)}: \mathcal{M}_{k} \times \prod_{j^{\prime}=1}^{j-1} \prod_{\tilde{k} \in\{k-1, k+1\}} & \left\{1, \ldots,\left\lfloor 2^{\left.\left.n R_{\mathrm{Tx}, \tilde{k} \rightarrow k}^{\left(j^{\prime}\right)}\right\rfloor\right\}}\right.\right. \\
& \longrightarrow\left\{1, \ldots,\left\lfloor 2^{n R_{\mathrm{Tx}, k \rightarrow k^{\prime}}^{(j)}}\right\rfloor\right\}
\end{aligned}
$$

and we understand $U_{0 \rightarrow 1}^{(j)}$ and $U_{K+1 \rightarrow K}^{(j)}$ to be degenerate random variables (constants) with

$$
R_{\mathrm{Tx}, 0 \rightarrow 1}^{(j)}=R_{\mathrm{Tx}, K+1 \rightarrow K}^{(j)}=0, \quad \forall j \in\left\{1, \ldots, \kappa_{\mathrm{Tx}}\right\} .
$$

The total rate from Tx $k$ to each one of its neighbours may not exceed the rate budget:

$$
\sum_{j=1}^{\kappa_{\mathrm{Tx}}} R_{\mathrm{Tx}, k \rightarrow k^{\prime}}^{(j)} \leq R_{\mathrm{Tx}}
$$

for all $k^{\prime} \in\{k-1, k+1\}$ and $k \in\{1, \ldots, K\}$.

\section{Cooperative-Communication Phase}

The channel codeword sent by Tx $k$,

$$
X_{k}^{n}=\left(X_{k, 1}, X_{k, 2}, \ldots, X_{k, n}\right),
$$

is a function of its source message $M_{k}$ and the conferencing messages it received during the Tx-conferencing phase. Specifically

$$
\begin{aligned}
& X_{k}^{n}:=f_{k}^{(n)}\left(M_{k}, \quad U_{k-1 \rightarrow k}^{(1)}, \quad U_{k+1 \rightarrow k}^{(1)}, U_{k-1 \rightarrow k}^{(2)},\right. \\
& \left.U_{k+1 \rightarrow k}^{(2)}, \ldots U_{k-1 \rightarrow k}^{\left(k_{\mathrm{Tx}}\right)}, \quad U_{k+1 \rightarrow k}^{\left(\kappa_{\mathrm{Tx}}\right)}\right),
\end{aligned}
$$

and

$$
\begin{array}{r}
f_{k}^{(n)}: \mathcal{M}_{k} \times \prod_{j=1}^{\kappa_{\mathrm{Tx}}} \prod_{k^{\prime} \in\{k-1, k+1\}}\left\{1, \ldots,\left\lfloor 2^{\left.\left.n R_{\mathrm{Tx}, k^{\prime} \rightarrow k}^{(j)}\right\rfloor\right\}}\right.\right. \\
\rightarrow \mathbb{R}^{n} .
\end{array}
$$

\section{E. Rx-Conferencing Phase With $\kappa_{R x}$ Rounds}

The Rx-conferencing phase takes place after all the channel outputs have been observed by the receivers. Let

$$
Y_{k}^{n}=\left(Y_{k, 1}, Y_{k, 2}, \ldots, Y_{k, n}\right)
$$

denote the channel outputs observed at receiver $k$.

The Rx-conferencing phase consists of $\kappa_{\mathrm{Rx}}$ rounds: In round $j, \operatorname{Rx} k$ sends a conferencing message $V_{k \rightarrow k^{\prime}}^{(j)}$ to its neighbour $\operatorname{Rx} k^{\prime}$, for $k^{\prime} \in\{k-1, k+1\}$. Here, the round- $j$ conferencing message from $\mathrm{Rx} k$ to $\mathrm{Rx} k^{\prime} \in\{k-$ $1, k+1\}$ is a function of the outputs $Y_{k}^{n}$ as well as all conferencing messages sent to $\mathrm{Rx} k$ in previous rounds $1,2, \ldots, j-1$ :

$$
\begin{array}{r}
V_{k \rightarrow k^{\prime}}^{(j)}:=\psi_{k, k^{\prime}}^{(j)}\left(Y_{k}^{n}, V_{k-1 \rightarrow k}^{(1)}, V_{k+1 \rightarrow k}^{(1)}, V_{k-1 \rightarrow k}^{(2)},\right. \\
\left.V_{k+1 \rightarrow k}^{(2)}, \ldots, V_{k-1 \rightarrow k}^{(j-1)}, V_{k+1 \rightarrow k}^{(j-1)}\right),
\end{array}
$$

where

$$
\begin{aligned}
\psi_{k, k^{\prime}}^{(j)}: \mathbb{R}^{n} \times \prod_{j^{\prime}=1}^{j-1} \prod_{\tilde{k} \in\{k-1, k+1\}} & \left\{1, \ldots,\left\lfloor 2^{\left.\left.n R_{\mathrm{Rx}, \tilde{k} \rightarrow k}^{\left(j^{\prime}\right)}\right\rfloor\right\}}\right.\right. \\
& \longrightarrow\left\{1, \ldots,\left\lfloor 2^{n R_{\mathrm{Rx}, k \rightarrow k^{\prime}}^{(j)}}\right\rfloor\right\}
\end{aligned}
$$

and we understand $V_{0 \rightarrow 1}^{(j)}$ and $V_{K+1 \rightarrow K}^{(j)}$ to be degenerate random variables (constants) with

$$
R_{\mathrm{Rx}, 0 \rightarrow 1}^{(j)}=R_{\mathrm{Rx}, K+1 \rightarrow K}^{(j)}=0, \quad \forall j \in\left\{1, \ldots, \kappa_{\mathrm{Rx}}\right\} .
$$

We require that the total rate from receiver $k$ to its immediate neighbours does not exceed the rate budget,

$$
\sum_{j=1}^{\kappa_{\mathrm{Rx}}} R_{\mathrm{Rx}, k \rightarrow k^{\prime}}^{(j)} \leq R_{\mathrm{Rx}}, \quad \forall k^{\prime} \in\{k-1, k+1\} .
$$

\section{F. Decoding Phase}

Receiver $k$ estimates the source message $M_{k}$ by

$$
\begin{aligned}
\hat{M}_{k}:=g_{k}\left(Y_{k}^{n}, V_{k-1 \rightarrow k}^{(1)}, V_{k+1 \rightarrow k}^{(1)}, V_{k-1 \rightarrow k}^{(2)},\right. \\
\left.V_{k+1 \rightarrow k}^{(2)}, \ldots, \quad V_{k-1 \rightarrow k}^{\left(\kappa_{\mathrm{Rx}}\right)}, V_{k+1 \rightarrow k}^{\left(\kappa_{\mathrm{Rx}}\right)}\right)
\end{aligned}
$$

where






\section{G. Capacity, Sum Capacity and Per-User Multiplexing Gain}

Let $\boldsymbol{\kappa}=\left(\kappa_{\mathrm{Tx}}, \kappa_{\mathrm{Rx}}\right), \boldsymbol{\mu}=\left(\mu_{\mathrm{Tx}}, \mu_{\mathrm{Rx}}\right)$ and $\boldsymbol{R}=\left(R_{1}, \ldots\right.$, $\left.R_{K}\right)$. We call the collection of encoders and decoders in (5), (7), (8) and (10) an $(n, \boldsymbol{R}, \boldsymbol{\kappa}, \boldsymbol{\mu}, P)$-code. For given $\boldsymbol{\kappa}, \boldsymbol{\mu}$ and $P$ : We say that a rate tuple $\boldsymbol{R}$ is $(\boldsymbol{\kappa}, \boldsymbol{\mu}, P)$-achievable if for every $\epsilon>0$ there exists a sufficiently large blocklength $n$ and an $(n, \boldsymbol{R}, \boldsymbol{\kappa}, \boldsymbol{\mu}, P)$-code with

$$
\mathbb{P}\left[\left(\hat{M}_{1}, \ldots, \hat{M}_{K}\right) \neq\left(M_{1}, \ldots, M_{K}\right)\right] \leq \epsilon .
$$

The capacity region $\mathrm{C}(\boldsymbol{\kappa}, \boldsymbol{\mu}, P)$ is the closure of the set of all $(\kappa, \mu, P)$-achievable rate tuples, and the sum capacity is

$$
\mathrm{C}_{\Sigma}(\boldsymbol{\kappa}, \boldsymbol{\mu}, P):=\sup _{\boldsymbol{R} \in \mathrm{C}(\kappa, \mu, P)} \sum_{k=1}^{K} R_{k} .
$$

Definition 1: The per-user multiplexing gain $(M G)$ is

$$
\mathrm{S}(\kappa, \boldsymbol{\mu}):=\varlimsup_{K \rightarrow \infty} \varlimsup_{P \rightarrow \infty} \frac{\mathrm{C}_{\Sigma}(\kappa, \mu, P)}{K \cdot \frac{1}{2} \log P},
$$

for $\boldsymbol{\kappa} \in \mathbb{Z}^{+} \times \mathbb{Z}^{+}$and $\boldsymbol{\mu} \in[0, \infty) \times[0, \infty)$.

The main problem of interest in this paper is to determine $\mathrm{S}(\kappa, \boldsymbol{\mu})$. In Section IV, we give upper and lower bounds on $\mathrm{S}(\boldsymbol{\kappa}, \boldsymbol{\mu})$ and show that these bounds coincide in many cases. The next proposition summarises some basic properties of the per-user MG, and we omit its proof.

Proposition 1:

1) $\mathrm{S}(\boldsymbol{\kappa}, \boldsymbol{\mu})$ is nondecreasing in $\boldsymbol{\kappa}$ and $\boldsymbol{\mu}$ and upper bounded by 1 .

2) If $\mu_{\mathrm{Tx}}=0$, then $\mathbf{S}(\boldsymbol{\kappa}, \boldsymbol{\mu})$ does not depend on $\kappa_{\mathrm{Tx}}$ :

$$
\mathrm{S}\left(0, \kappa_{\mathrm{Rx}}, 0, \mu_{\mathrm{Rx}}\right)=\mathrm{S}\left(1, \kappa_{\mathrm{Rx}}, 0, \mu_{\mathrm{Rx}}\right)=\cdots
$$

for all $\kappa_{\mathrm{Rx}} \in \mathbb{Z}^{+}$and $\mu_{\mathrm{Rx}} \in[0, \infty)$.

Similarly, if $\mu_{\mathrm{Rx}}=0$, then $\mathbf{S}(\kappa, \boldsymbol{\mu})$ does not depend on $\kappa_{\mathrm{Rx}}$ :

$$
\mathrm{S}\left(\kappa_{\mathrm{Tx}}, 0, \mu_{\mathrm{Tx}}, 0\right)=\mathrm{S}\left(\kappa_{\mathrm{Tx}}, 1, \mu_{\mathrm{Tx}}, 0\right)=\cdots
$$

for all $\kappa_{\mathrm{Tx}} \in \mathbb{Z}^{+}$and $\mu_{\mathrm{Tx}} \in[0, \infty)$.

3) If $\boldsymbol{\mu}=(0,0)$, then

$$
\mathrm{S}\left(\kappa_{\mathrm{Tx}}, \kappa_{\mathrm{Rx}}, 0,0\right)=\frac{1}{2}
$$

for all $\left(\kappa_{\mathrm{Tx}}, \kappa_{\mathrm{Rx}}\right) \in \mathbb{Z}^{+} \times \mathbb{Z}^{+}$.

\section{H. Conferencing With Unlimited Rounds}

To help put our results $S(\kappa, \mu)$ in context, we will also consider the case of unlimited $\mathrm{Tx}$ - and $\mathrm{Rx}$-conferencing rounds. The per-user $M G$ with unlimited conferencing is defined in the same way as above, except now $\kappa_{\mathrm{Tx}}$ and $\kappa_{\mathrm{Rx}}$ are infinite. ${ }^{1}$ Let $S_{\infty}(\boldsymbol{\mu})$ denote the per-user MG with unlimited conferencing. The next proposition summarises some basic properties of $\mathrm{S}_{\infty}(\boldsymbol{\mu})$, and we omit its proof.

\footnotetext{
${ }^{1}$ For a given blocklength $n$ scheme, it however suffices to choose $\kappa_{\mathrm{Tx}} \leq$ $2 K n R_{\mathrm{Tx}}$ and $\kappa_{\mathrm{Rx}} \leq 2 K n R_{\mathrm{Rx}}$, because each of the $K$ transmitters can send at most $n R_{\mathrm{Tx}}$ bits over each of the two links to its left and right, and similarly each of the $K$ receivers can send at most $n R_{\mathrm{Rx}}$ bits over each of the two links to its left and right.
}

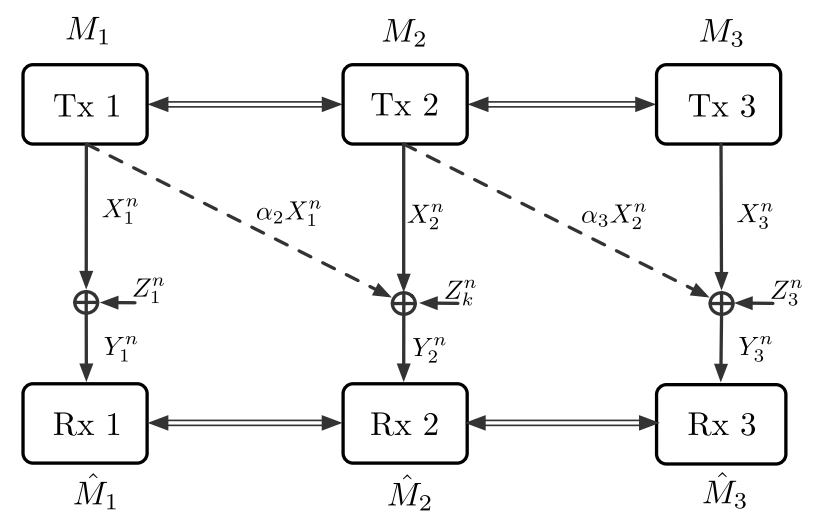

Fig. 2. An example network with $K=3$ transmitter and receiver pairs.

\section{Proposition 2:}

1) $S_{\infty}(\boldsymbol{\mu})$ is nondecreasing in $\boldsymbol{\mu}$.

2) For all $\kappa \in \mathbb{Z}^{+} \times \mathbb{Z}^{+}$and $\mu \in[0, \infty) \times[0, \infty)$ :

$$
\frac{1}{2} \leq \mathrm{S}(\boldsymbol{\kappa}, \boldsymbol{\mu}) \leq \mathrm{S}_{\infty}(\boldsymbol{\mu}) \leq 1
$$

\section{AN EXAMPLE}

To help illustrate how the number of conferencing rounds and the choice of conferenced signals influences the peruser MG, consider the simple network with 3 transmitters and 3 receivers in Figure 2. For simplicity we focus on the two cases with only transmitter-conferencing from left-to-right (Subsection III-A) and with only receiver-conferencing from left-to-right (Subsection III-B).

\section{A. Transmitter-Conferencing From Left-to-Right}

Suppose that each transmitter can send conferencing messages only to its right neighbour and the receivers cannot send any conferencing messages at all, see Figure 3.

Most previous results on transmitter-conferencing used a data-sharing approach, where the transmitters transmit and relay parts of source messages over the conferencing links [2], [3], [5]-[7]. Recently, Ntranos et al. [24] suggested a compression approach for the conferencing phase, where the transmitters use the conferencing links to describe quantised versions of their transmit signals. As we illustrate at hand of the following example, both approaches facilitate interference cancellation at the transmitters (through dirty-paper coding or precoding). The data-sharing approach, however, requires larger conferencing prelogs.

Figure 4 illustrates the data-sharing approach: Each transmitter sends the messages it is cognizant of over the conferencing link to its right neighbour. After conferencing each transmitter can produce its channel inputs as a function of its own message and the input signal of its leftneighbour, and, therefore, it can mitigate the interference at its corresponding receiver. The scheme achieves full asymptotic multiplexing-gain per-user 1 under the following two conditions:

- The conferencing-prelog is at least 2: Tx 2 has to send two messages of prelog 1 to Tx 3 . 


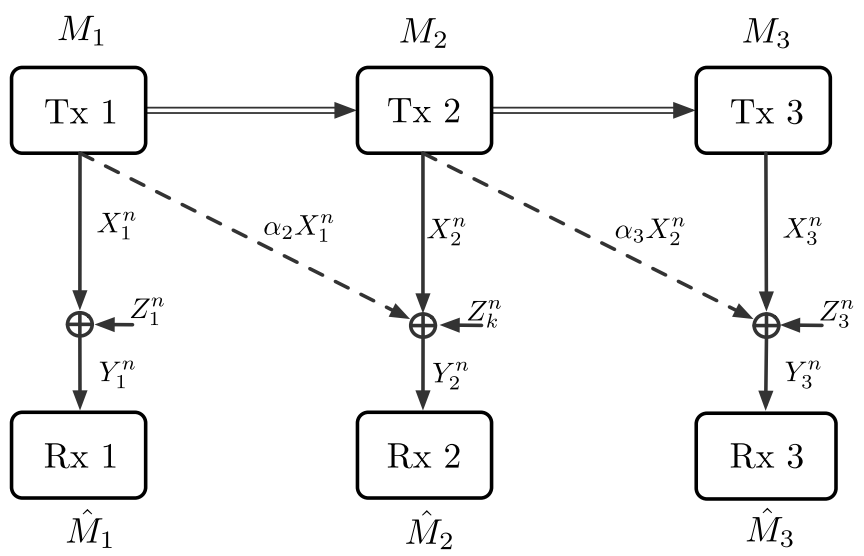

Fig. 3. Example with transmitter-conferencing from left-to-right.



Fig. 4. Tx 1 sends $M_{1}$ to Tx 2 , and Tx 2 sends the pair $\left(M_{1}, M_{2}\right)$ to Tx 3.

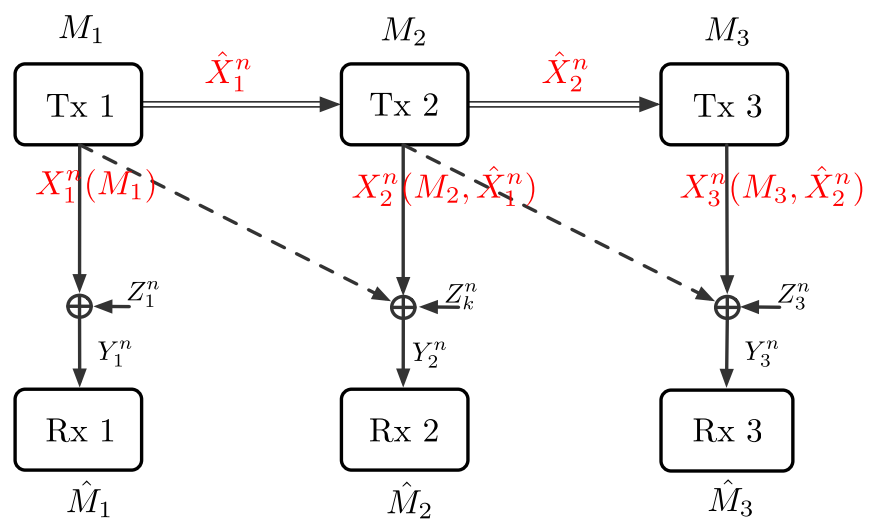

Fig. 5. Tx 1 sends the quantized version $\hat{X}_{1}^{n}$ of its inputs $X_{1}^{n}$ to Tx 2, and Tx 2 the quantized version $\hat{X}_{2}^{n}$ of its inputs $X_{2}^{n}$ to Tx 3 .

- Conferencing takes place over at least 2 rounds: Tx 2 has to wait until conferencing round 2 before it can send $M_{1}$ to $\mathrm{Tx} 3$.

Figure 5 illustrates the compression-approach: Each transmitter sends a compressed version of its input signal over the conferencing link to its right neighbour. This right neighbouring transmitter can mitigate some of the interference experienced at its corresponding receiver. The scheme achieves full asymptotic multiplexing-gain per-user 1 under the following two conditions:

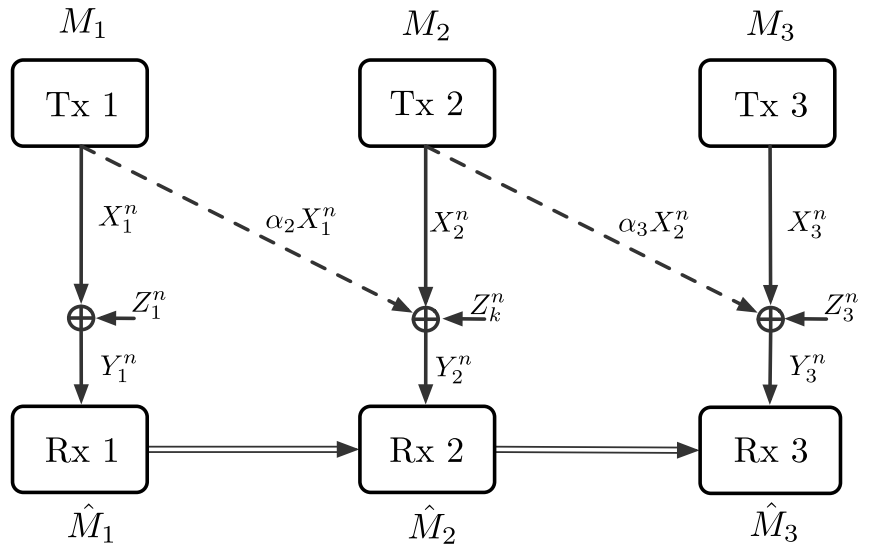

Fig. 6. An example network with $K=3$ transmitter and receiver pairs Conferencing links exist only from Rx 1 to Rx 2 and from Rx 2 to Rx 3.

- The conferencing-prelog is at least 1 . It suffices to choose the quantisation level so that the quantisation noise is below the noise level.

- Conferencing takes place over at least 2 rounds: Tx 2 has to wait until after conferencing round 1 before it can compute $X_{2}^{n}$ and send $\hat{X}_{2}^{n}$ to Tx 3 .

Therefore, in our example with $K=3$ transmitter/receiver pairs, 2 rounds of conferencing are required to achieve full per-user MG 1. Moreover conferencing prelog 1 suffices if the transmitters conference quantised input signals. With a single conferencing round only a per-user MG of $2 / 3$ is achievable, irrespective of the conferencing prelog.

More generally, in Wyner's asymmetric network with $K$ transmitter/receiver pairs, $K-1$ conferencing rounds are required to achieve full asymptotic MG per-user 1 if each transmitter can only conference with the neighbouring transmitter to its right. Similar conclusions hold also for transmitterconferencing from right-to-left.

\section{B. Receiver Conferencing From Left-to-Right}

Now suppose that each receiver can send conferencing messages only to its right neighbour and the transmitters cannot send any conferencing messages at all, see Figure 6. Two main approaches have been proposed in the literature for receiver conferencing. In the compression approach [8]-[10] receivers quantize their channel outputs and send quantization messages over the conferencing links. In the data-sharing approach [8], [9], [12], [13] receivers decode some of the transmitted messages and then send these decoded messages over the conferencing links. As we will see shortly at hand of our example, both approaches allow the receivers to mitigate interference through successive-interference cancellation.

Figure 7 illustrates the compression approach: Each receiver compresses its output signal and sends all compression indices that it knows over the conferencing link to its right neighbour. So Rx 1 sends the compression index of $\hat{Y}_{1}^{n}$ to Rx 2, which relays this information on to to $\mathrm{Rx} 3$. Rx 2, moreover, also sends the compression index of $\hat{Y}_{2}^{n}$ to Rx 3 .

Rx 1 decodes its desired message $M_{1}$ based on its interference-free outputs $Y_{1}^{n}$. Rx 2 first decodes $M_{1}$ from $\hat{Y}_{1}^{n}$, 




Fig. 7. Rx 1 sends the quantized version $\hat{Y}_{1}^{n}$ of its outputs $Y_{1}^{n}$ to $\mathrm{Rx} 2$, which relays them also to Rx 3. Rx 2 also sends the quantized version $\hat{Y}_{2}^{n}$ of its outputs $Y_{2}^{n}$ to $\mathrm{Rx} 3$.

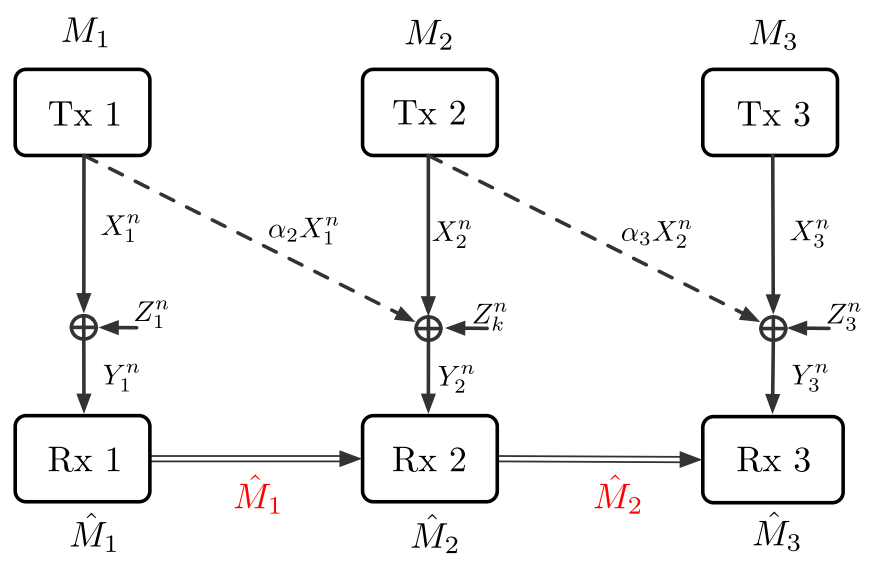

Fig. 8. Rx 1 sends its guess $\hat{M}_{1}$ to $\mathrm{Rx} 2$, and $\mathrm{Rx} 2$ sends the guess $\hat{M}_{2}$ to $\operatorname{Rx} 3$.

then reconstructs $X_{1}^{n}\left(\hat{M}_{1}\right)$ with its guess $\hat{M}_{1}$, and decodes $M_{2}$ based on the difference $Y_{2}^{n}-\alpha_{2} X_{1}^{n}\left(\hat{M}_{1}\right)$. Rx 3 also starts by decoding $M_{1}$ from $\hat{Y}_{1}^{n}$, then decodes $M_{2}$ based on the difference $\hat{Y}_{2}^{n}-\alpha_{2} X_{1}^{n}\left(\hat{M}_{1}\right)$ and finally decodes $M_{3}$ from $Y_{3}^{n}-\alpha_{3} X_{2}^{n}\left(\hat{M}_{2}\right)$ where $\hat{M}_{2}$ denotes its guess of $M_{2}$. This scheme achieves full per-user MG 1 under the following two conditions:

- The conferencing-prelog is at least 2: Each quantisation index needs to be of prelog 1 so as to assure that the quantisation noise be below the noise level, and $\mathrm{Rx} 2$ sends two quantisation messages describing $\hat{Y}_{1}$ and $\hat{Y}_{2}$ to Rx 3.

- Conferencing takes place over at least 2 rounds: $\mathrm{Rx} 2$ has to wait until after conferencing round 1 before it can relay $\hat{Y}_{1}^{n}$ to $\mathrm{Rx} 3$.

Figure 8 illustrates the data-sharing approach: Each receiver sends its own decoded message over the conferencing link to its right neighbour. As a consequence, after the conferencing each receiver can reconstruct and subtract the sequence interfering its outputs. Thus, Rxs 2 and 3 can decode their desired messages based on the difference $Y_{2}^{n}-\alpha_{2} X_{1}^{n}\left(\hat{M}_{1}\right)$ and $Y_{3}^{n}-\alpha_{3} X_{2}^{n}\left(\hat{M}_{2}\right)$, respectively. Notice that whenever they obtained the correct guesses $\hat{M}_{1}$ and $\hat{M}_{2}$ over the conferencing links, then they can decode based on interference-free outputs.

The described scheme achieves full asymptotic multiplexing-gain per-user 1 under the following two conditions:

- The conferencing-prelog is at least 1: Each receiver sends a guess of its prelog-1 message to its right neighbour.

- Conferencing can take place over at least 2 rounds: Rx 2 has to wait until conferencing round 2 before it can send $\hat{M}_{2}$ to receiver 3 , because it calculates $\hat{M}_{2}$ based on it outputs $Y_{2}^{n}$ and the round-1 message $\hat{M}_{1}$.

One can make similar observations as in the case with transmitter-conferencing only. That means, 2 rounds of conferencing are required to achieve full per-user MG 1, and conferencing-prelog 1 suffices if the receivers conference decoded messages. With a single conferencing round only a per-user MG of $2 / 3$ is achievable, irrespective of the conferencing prelog.

Generally, with $K$ transmitter/receiver pairs, $K-1$ conferencing rounds are required to achieve full per-user MG 1 if each receiver can only conference with the neighbouring receiver to its right. Similar conclusions hold also for receiverconferencing from right-to-left.

\section{MAin Results}

\section{A. Conferencing With Unlimited Number of Rounds}

First consider the case where the number of conferencing rounds is unconstrained. The next theorem determines the exact per-user MG.

Theorem 1:

$$
\mathrm{S}_{\infty}(\boldsymbol{\mu})=\min \left\{1, \frac{1+2 \mu_{\mathrm{Tx}}+2 \mu_{\mathrm{Rx}}}{2}\right\}
$$

for all $\boldsymbol{\mu} \in[0, \infty) \times[0, \infty)$.

Since there is no limitation on the number of conferencing rounds, every conferencing prelog is useful and increases the per-user MG, irrespective of whether the prelog pertains to transmitter- or to receiver-conferencing or whether it pertains to left-to-right or to right-to-left conferencing. In other words, the per-user MG grows linearly in the sum of all prelogs $2\left(\mu_{\mathrm{Tx}}+\mu_{\mathrm{Rx}}\right)$ until it reaches the maximum possible value 1 .

Proof of Theorem 1: The proof of Theorem 1 consists of a direct part proving

$$
\mathrm{S}_{\infty}(\boldsymbol{\mu}) \geq \min \left\{1, \frac{1+2 \mu_{\mathrm{Tx}}+2 \mu_{\mathrm{Rx}}}{2}\right\}
$$

and a converse part proving

$$
\mathrm{S}_{\infty}(\boldsymbol{\mu}) \leq \min \left\{1, \frac{1+2 \mu_{\mathrm{Tx}}+2 \mu_{\mathrm{Rx}}}{2}\right\} .
$$

The converse part is proved in Section VI.

The direct part follows from Theorem 2, which is stated in the next section, and from continuity considerations. Specifically, for

$$
\mu_{\mathrm{Tx}}+\mu_{\mathrm{Rx}}<\frac{1}{2},
$$


the desired per-user MG of $\left(1+2 \mu_{\mathrm{Tx}}+2 \mu_{\mathrm{Rx}}\right) / 2$ is achievable by Theorem 2 when one chooses the number of conferencing rounds $\kappa_{\mathrm{Tx}}$ and $\kappa_{\mathrm{Rx}}$ sufficiently large so that

$$
2 \mu_{\mathrm{Tx}}+2 \mu_{\mathrm{Rx}}+2 \min \left\{\frac{\mu_{\mathrm{Tx}}}{\kappa_{\mathrm{Tx}}}, \frac{\mu_{\mathrm{Rx}}}{\kappa_{\mathrm{Rx}}}\right\}<1 .
$$

(Since here the number of conferencing rounds is unlimited, we can choose $\kappa_{\mathrm{Tx}}$ and $\kappa_{\mathrm{Rx}}$ as large as we wish.) Moreover, since the per-user MG is non-decreasing in $\mu_{\mathrm{Tx}}$ and $\mu_{\mathrm{Rx}}$ (see Proposition 1) a per-user MG of 1 must be achievable whenever

$$
\mu_{\mathrm{Tx}}+\mu_{\mathrm{Rx}} \geq \frac{1}{2} .
$$

\section{B. Conferencing With Finite Rounds}

We now consider the case where the number of transmitterconferencing rounds is constrained to $\kappa_{\mathrm{Tx}}$ and the number of receiver-conferencing rounds is constrained to $\kappa_{\mathrm{Rx}}$. The next theorem determines the exact per-user MG $\boldsymbol{S}(\boldsymbol{\kappa}, \boldsymbol{\mu})$. Its expression depends on the following two quantities

$$
\pi_{\mathrm{Tx}}:=\frac{\mu_{\mathrm{Tx}}}{\kappa_{\mathrm{Tx}}} \text { and } \pi_{\mathrm{Rx}}:=\frac{\mu_{\mathrm{Rx}}}{\kappa_{\mathrm{Rx}}} .
$$

Define $S^{\star}: \mathbb{Z}^{+} \times \mathbb{Z}^{+} \times[0, \infty) \times[0, \infty) \rightarrow[0,1]$ as follows: If $\pi_{\mathrm{Rx}}=\pi_{\mathrm{Tx}}$, let

$S^{\star}(\boldsymbol{\kappa}, \boldsymbol{\mu})= \begin{cases}\frac{2 \mu_{\mathrm{Tx}}+2 \mu_{\mathrm{Rx}}+1}{2} & \text { if } 2 \mu_{\mathrm{Tx}}+2 \mu_{\mathrm{Rx}}+2 \pi_{\mathrm{Tx}} \leq 1 \\ \frac{2 \kappa_{\mathrm{Tx}}+2 \kappa_{\mathrm{Rx}}+1}{2 \kappa_{\mathrm{Tx}}+2 \kappa_{\mathrm{Rx}}+2} & \text { otherwise. }\end{cases}$

If $\pi_{\mathrm{Rx}}<\pi_{\mathrm{Tx}}$, let

$\mathbf{S}^{\star}(\boldsymbol{\kappa}, \boldsymbol{\mu})=\left\{\begin{array}{cc}\frac{2 \mu_{\mathrm{Tx}}+2 \mu_{\mathrm{Rx}}+1}{2} & \text { if } 2 \mu_{\mathrm{Tx}}+2 \mu_{\mathrm{Rx}}+2 \pi_{\mathrm{Tx}} \leq 1 \\ \frac{2 \kappa_{\mathrm{Tx}}+2 \kappa_{\mathrm{Rx}}+1}{2 \kappa_{\mathrm{Tx}}+2 \kappa_{\mathrm{Rx}}+2} & \text { if } 2 \mu_{\mathrm{Tx}}\left(\frac{\pi_{\mathrm{Rx}}}{\pi_{\mathrm{Tx}}}\right) \\ & +2 \mu_{\mathrm{Rx}}+2 \pi_{\mathrm{Rx}}>1 \\ \frac{2 \kappa_{\mathrm{Tx}}+2 \mu_{\mathrm{Rx}}+1}{2 \kappa_{\mathrm{Tx}}+2} & \text { otherwise. }\end{array}\right.$

$$
\begin{aligned}
& \text { If } \pi_{\mathrm{Rx}}>\pi_{\mathrm{Tx}}, \text { let } \\
& \mathrm{S}^{\star}(\boldsymbol{\kappa}, \boldsymbol{\mu})=\left\{\begin{array}{cc}
\frac{2 \mu_{\mathrm{Tx}}+2 \mu_{\mathrm{Rx}}+1}{2} & \text { if } 2 \mu_{\mathrm{Tx}}+2 \mu_{\mathrm{Rx}}+2 \pi_{\mathrm{Rx}} \leq 1 \\
\frac{2 \kappa_{\mathrm{Tx}}+2 \kappa_{\mathrm{Rx}}+1}{2 \kappa_{\mathrm{Tx}}+2 \kappa_{\mathrm{Rx}}+2} & \text { if } 2 \mu_{\mathrm{Tx}}+2 \mu_{\mathrm{Rx}}\left(\frac{\pi_{\mathrm{Tx}}}{\pi_{\mathrm{Rx}}}\right) \\
& +2 \pi_{\mathrm{Tx}}>1 \\
\frac{2 \kappa_{\mathrm{Rx}}+2 \mu_{\mathrm{Tx}}+1}{2 \kappa_{\mathrm{Rx}}+2} & \text { otherwise. }
\end{array}\right.
\end{aligned}
$$

Theorem 2: For all $\boldsymbol{\kappa} \in \mathbb{Z}^{+} \times \mathbb{Z}^{+}$and $\boldsymbol{\mu} \in[0, \infty) \times[0, \infty)$, we have

$$
\mathrm{S}(\kappa, \mu)=\mathrm{S}^{\star}(\kappa, \mu) .
$$

Proof: The achievability is proved in Section V. The converse in Section VI.

Remark 2: The coding scheme that we present in Section V requires that the codebooks are known during the $\mathrm{Tx}$ - and Rx-conferencing phases. When $\kappa_{\mathrm{Tx}}=\kappa_{\mathrm{Rx}}=1$, then it is possible to find a coding scheme achieving $S^{\star}(\boldsymbol{\kappa}, \boldsymbol{\mu})$ where the conferencing phases do not use knowledge about codebooks. Our results for $\kappa_{\mathrm{Tx}}=\kappa_{\mathrm{Rx}}=1$ thus continue to hold also in oblivious setups. The scheme in Section V needs to be changed as follows: Instead of sending quantised versions of transmit signals over the Tx-conferencing links, the transmitters conference source messages, and instead of sending decoded sources messages over the Rx-conferencing links, the receivers send quantised versions of their receive signals. (Details omitted.)

Without conferencing, $\mu_{\mathrm{Rx}}=\mu_{\mathrm{Tx}}=0$, the per-user MG $S(\kappa, \mu)$ cannot exceed $1 / 2$ (which is also achievable). The reason is that without conferencing every second transmitter needs to be switched off so as to avoid interference to propagate. When conferencing is possible, $\mu_{\mathrm{Tx}}, \mu_{\mathrm{Rx}}>0$, then the transmitters can precancel interference at their intended receivers using dirtypaper coding or simple precoding, and receivers can cancel interference using successive interference cancellation. The bound

$$
\mathrm{S}(\boldsymbol{\kappa}, \boldsymbol{\mu}) \leq\left(1+2 \mu_{\mathrm{Tx}}+2 \mu_{\mathrm{Rx}}\right) / 2
$$

indicates that to cancel interference of rate $\zeta$, either the transmitter or the receiver need side-information of rate $\zeta$.

The bound

$$
\mathrm{S}(\boldsymbol{\kappa}, \boldsymbol{\mu}) \leq \frac{2 \kappa_{\mathrm{Tx}}+2 \kappa_{\mathrm{Rx}}+1}{2 \kappa_{\mathrm{Tx}}+2 \kappa_{\mathrm{Rx}}+2}
$$

captures the fact that per set of $2 \kappa_{\mathrm{Tx}}+2 \kappa_{\mathrm{Rx}}+2$ consecutive transmitter/receiver pairs the total MG cannot exceed $2 \kappa_{\mathrm{Tx}}+2 \kappa_{\mathrm{Rx}}+1$. Intuitively, this is because with $\kappa_{\mathrm{Tx}}$ rounds of left-to-right and right-to-left transmitter-conferencing and $\kappa_{\mathrm{Rx}}$ rounds of left-to-right and right-to-left receiver-conferencing information about source messages or output signals cannot propagate beyond a subset of $2 \kappa_{\mathrm{Tx}}+2 \kappa_{\mathrm{Rx}}+1$ transmitter/receiver pairs, which makes it impossible to cancel interference at more than $2 \kappa_{\mathrm{Tx}}+2 \kappa_{\mathrm{Rx}}+1$ consecutive transmitter/receiver pairs. (See also Section III for more explanations on the special cases with only left-toright transmitter-conferencing or only left-to-right receiverconferencing.)

Example 1: Let $\boldsymbol{\kappa}=(\kappa, \kappa)$ and $\boldsymbol{\mu}=(\mu, \mu / 2)$. In this case $\mathrm{S}(\kappa, \kappa, \mu, \mu / 2)$ is given by (16b). Figure 9 plots (16b) as a function of $\mu$ for $\kappa=1,2$ and 3 conferencing rounds. The figure also shows the per-user MG with unlimited conferencing 


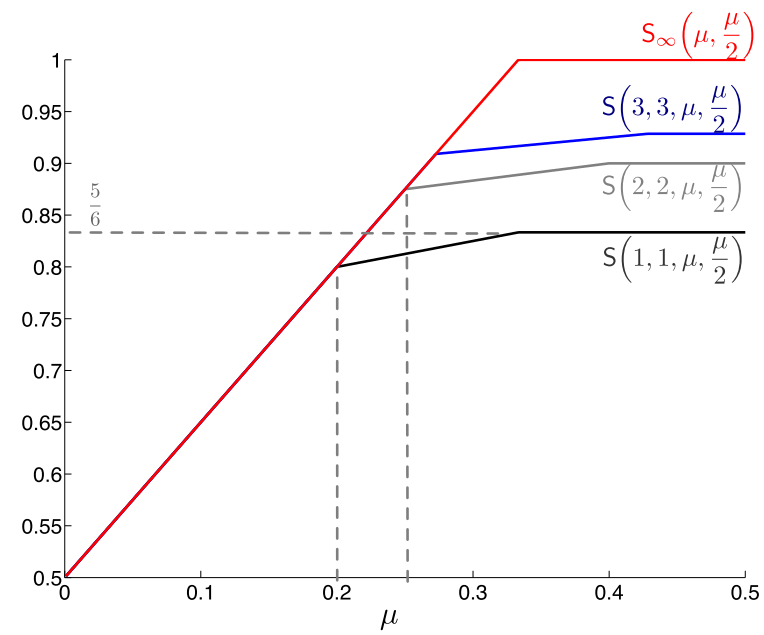

Fig. 9. The exact per-user MG $S(\kappa, \mu)$ for the case with equal Tx- and Rx-conferencing rounds $\kappa=(\kappa, \kappa)$ and unbalanced $\mathrm{Tx}$ and $\mathrm{Rx}$ prelog conferencing contraints $\mu_{\mathrm{Tx}}=2 \mu_{\mathrm{Rx}}$. The per-user MG for unlimited conferencing rounds $\mathrm{S}_{\infty}(\boldsymbol{\mu})$, as described in Theorem 1, is also shown.

rounds from Theorem 1,

$$
\mathrm{S}_{\infty}\left(\mu, \frac{\mu}{2}\right)= \begin{cases}\frac{3 \mu+1}{2} & \text { if } 0 \leq \mu \leq \frac{1}{3} \\ 1 & \text { otherwise. }\end{cases}
$$

From Figure 9 we differentiate three regimes:

- Small $\mu$ region: When the prelog constant $\mu$ is small, $\mu \in$ $[0,1 / 5]$, then a single conferencing round achieves the same per-user MG as with multiple or unlimited number of rounds:

$$
\begin{aligned}
\mathrm{S}_{\infty}\left(\mu, \frac{\mu}{2}\right) & =\mathrm{S}\left(1,1, \mu, \frac{\mu}{2}\right)=\mathrm{S}\left(2,2, \mu, \frac{\mu}{2}\right)=\ldots \\
& =\frac{3 \mu+1}{2}, \quad \mu \in[0,1 / 5] .
\end{aligned}
$$

- Moderate $\boldsymbol{\mu}$ region: When the prelog constant $\mu$ is moderate, $\mu \in(1 / 5,1 / 3)$, then a single conferencing round is strictly suboptimal. Multiple, but still finitely many, conferencing rounds are needed to attain the same per-user MG as with an unlimited number of rounds. For example, two conferencing rounds are sufficient when $\mu \in(1 / 5,1 / 4]:$

$$
\begin{aligned}
\mathrm{S}_{\infty}\left(\mu, \frac{\mu}{2}\right) & =\mathrm{S}\left(2,2, \mu, \frac{\mu}{2}\right)=\mathrm{S}\left(3,3, \mu, \frac{\mu}{2}\right)=\ldots \\
& =\frac{3 \mu+1}{2} \\
& >S\left(1,1, \mu, \frac{\mu}{2}\right)=\frac{\mu+3}{4} .
\end{aligned}
$$

- Large $\boldsymbol{\mu}$ region: When the prelog constant $\mu$ is large, $\mu \geq 1 / 3$, then with an unlimited number of conferencing rounds the per-user $\mathrm{MG}$ is 1 , so equal to its maximum value. This per-user $M G$ is not attainable with any finite number of conferencing rounds or any finite conferencing prelog $\mu$. In fact, with $\kappa$ conferencing rounds the per-user $\mathrm{MG}$

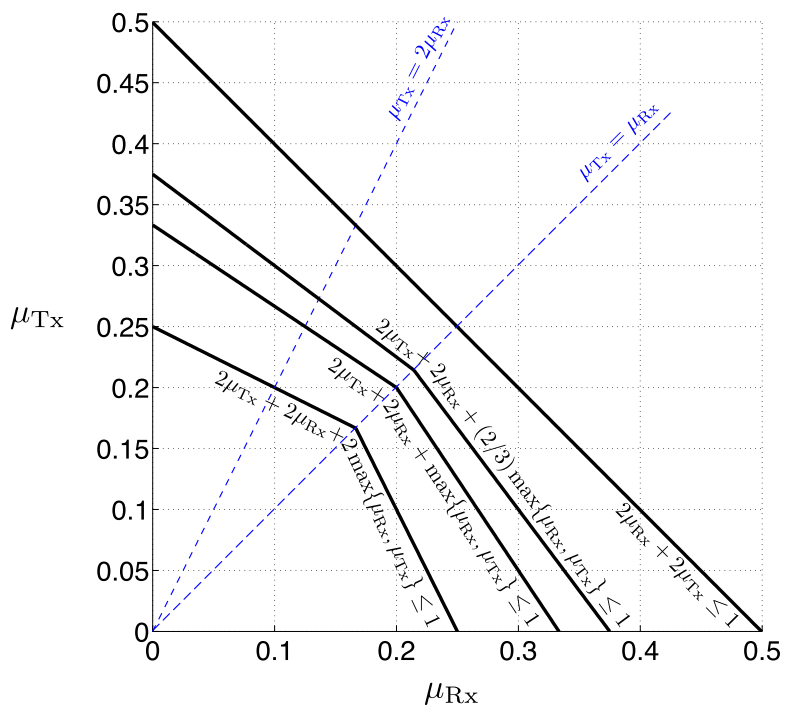

Fig. 10. An illustration of the small, moderate and large $\boldsymbol{\mu}$ regions assuming that $\kappa_{\mathrm{Tx}}=\kappa_{\mathrm{Rx}}$. The figure also shows the boundaries of the $\boldsymbol{\mu}$ regions where two or three conferencing rounds suffice.

saturates at

$$
\mathrm{S}\left(\kappa, \kappa, \mu, \frac{\mu}{2}\right)=\frac{4 \kappa+1}{4 \kappa+2}, \quad \text { for } \mu \geq \frac{\kappa}{2 \kappa+1} .
$$

As shown by the following two corollaries, the three $\boldsymbol{\mu}$ regions described for above example 1 and their typical behaviours arise also in general setups with arbitrary conferencing parameters $\boldsymbol{\kappa}, \boldsymbol{\mu}$. The corollaries are obtained by inspecting the expression for $S^{\star}(\kappa, \mu)$ in (16).

Corollary 1 (Small and Moderate $\mu$ Regions): Fix the number of Tx- and Rx-conferencing rounds $\kappa=\left(\kappa_{\mathrm{Tx}}, \kappa_{\mathrm{Rx}}\right)$. If the prelog conferencing constants $\boldsymbol{\mu}=\left(\mu_{\mathrm{Tx}}, \mu_{\mathrm{Rx}}\right)$ are small (depending on $\boldsymbol{\kappa}$ ), namely,

$$
2 \mu_{\mathrm{Tx}}+2 \mu_{\mathrm{Rx}}+2 \max \left\{\frac{\mu_{\mathrm{Tx}}}{\kappa_{\mathrm{Tx}}}, \frac{\mu_{\mathrm{Rx}}}{\kappa_{\mathrm{Rx}}}\right\} \leq 1,
$$

then $\boldsymbol{\kappa}$ conferencing rounds achieve the same performance as unlimited conferencing rounds; that is,

$$
\mathrm{S}(\boldsymbol{\kappa}, \boldsymbol{\mu})=\mathrm{S}\left(\boldsymbol{\kappa}^{\prime}, \boldsymbol{\mu}\right)=\mathrm{S}_{\infty}(\boldsymbol{\mu})=\frac{1+2 \mu_{\mathrm{Tx}}+2 \mu_{\mathrm{Rx}}}{2}
$$

for all $\kappa^{\prime} \in \mathbb{Z}^{+} \times \mathbb{Z}^{+}$with $\kappa_{\mathrm{Tx}} \leq \kappa_{\mathrm{Tx}}{ }^{\prime}$ and $\kappa_{\mathrm{Rx}} \leq \kappa_{\mathrm{Rx}}{ }^{\prime}$.

Corollary 2 (Large $\boldsymbol{\mu}$ Region): Fix the number of Tx- and $\mathrm{Rx}$ - conferencing rounds $\boldsymbol{\kappa}=\left(\begin{array}{ll}\kappa_{\mathrm{Tx}} & \kappa_{\mathrm{Rx}}\end{array}\right)$. If the prelog conferencing constants $\boldsymbol{\mu}=\left(\mu_{\mathrm{Tx}} \mu_{\mathrm{Rx}}\right)$ are sufficiently large (depending on $\boldsymbol{\kappa}$ ), namely,

$$
\begin{aligned}
\mu_{\mathrm{Tx}} \min \left\{1, \frac{\pi_{\mathrm{Rx}}}{\pi_{\mathrm{Tx}}}\right\}+\mu_{\mathrm{Rx}} \min \{1, & \left.\frac{\pi_{\mathrm{Tx}}}{\pi_{\mathrm{Rx}}}\right\} \\
& +\min \left\{\pi_{\mathrm{Tx}}, \pi_{\mathrm{Rx}}\right\}>\frac{1}{2},
\end{aligned}
$$

then,

$$
\mathrm{S}(\boldsymbol{\kappa}, \boldsymbol{\mu})=\frac{2 \kappa_{\mathrm{Tx}}+2 \kappa_{\mathrm{Rx}}+1}{2 \kappa_{\mathrm{Tx}}+2 \kappa_{\mathrm{Rx}}+2} .
$$

Figure 10 illustrates the regions of small, moderate and large $\mu$ 's under the assumption that $\kappa_{\mathrm{Tx}}=\kappa_{\mathrm{Rx}}$. Specifically, the 
No. of channel uses

\begin{tabular}{|c|c|c|c|c|c|c|c|c|c|c|}
\hline 1 & 2 & $\cdot \cdot$ & $N_{1}$ & $N_{1}+1$ & $N_{1}+2$ & $\cdot \cdot$ & $N_{1}+N_{2}$ & $N_{1}+N_{2}+1$ & $\cdot \cdot$ & $N_{1}+N_{2}+N_{3}$ \\
\hline
\end{tabular}

Fig. 11. Timesharing between Tx- and/or Rx-conferencing schemes.

first line $\left(2 \mu_{\mathrm{Tx}}+2 \mu_{\mathrm{Rx}}+\max \left\{\mu_{\mathrm{Tx}}, \mu_{\mathrm{Rx}}\right\} \leq 1\right)$ depicts the boundary of the small $\mu$ region where one-shot conferencing $\left(\kappa_{\mathrm{Tx}}=\kappa_{\mathrm{Rx}}=1\right)$ is optimal, i.e., achieves the same peruser MG as unlimited-rounds conferencing. The top-most line depicts the boundary of the large $\boldsymbol{\mu}$ region where with unlimited conferencing rounds the per-user MG saturates at 1 .

The second line $\left(2 \mu_{\mathrm{Tx}}+2 \mu_{\mathrm{Rx}}+\max \left\{\mu_{\mathrm{Tx}}, \mu_{\mathrm{Rx}}\right\} \leq 1\right)$ depicts the boundary of the $\boldsymbol{\mu}$ region where two-round conferencing $\left(\kappa_{\mathrm{Tx}}=\kappa_{\mathrm{Rx}}=2\right)$ is optimal, and the third line $\left(2 \mu_{\mathrm{Tx}}+\right.$ $\left.2 \mu_{\mathrm{Rx}}+(2 / 3) \max \left\{\mu_{\mathrm{Tx}}, \mu_{\mathrm{Rx}}\right\} \leq 1\right)$ depicts the boundary of the $\mu$ region where three-round conferencing $\left(\kappa_{\mathrm{Tx}}=\kappa_{\mathrm{Rx}}=3\right)$ is optimal. The left-most blue dashed line $\left(\mu_{\mathrm{Tx}}=2 \mu_{\mathrm{Rx}}=\mu\right)$ corresponds to the example in figure 9: its crossing point with the $2 \mu_{\mathrm{Tx}}+2 \mu_{\mathrm{Rx}}+\max \left\{\mu_{\mathrm{Tx}}, \mu_{\mathrm{Rx}}\right\} \leq 1$-line, for example, shows that $\kappa_{\mathrm{Tx}}=\kappa_{\mathrm{Rx}}=1$ is optimal for all $\mu \in[0,1 / 5]$.

The blue dashed line more to the right corresponds to the fully symmetric case $\mu_{\mathrm{Tx}}=\mu_{\mathrm{Rx}}=\mu$ and $\kappa_{\mathrm{Tx}}=\kappa_{\mathrm{Rx}}=\kappa$. For this setup, we now characterize analytically the minimum number of conferencing rounds $\kappa^{\star}$ that is required to attain the same per-user MG as with unlimited conferencing. Notice that $\kappa^{\star}$ only exists when $\mu \in[0,1 / 4]$. For $\mu \in[0,1 / 4]$, define

$$
\kappa^{\star}(\mu):=\min _{\kappa}\left\{\kappa \in \mathbb{Z}: \mathbf{S}(\kappa, \kappa, \mu, \mu)=\mathbf{S}_{\infty}(\mu, \mu)\right\} .
$$

Proposition 3 (Sufficient Conferencing Rounds With Equally-Strong Transmitter- and Receiver-Conferencings): If $\mu_{\mathrm{Tx}}=\mu_{\mathrm{Rx}}=\mu$ and $\kappa_{\mathrm{Tx}}=\kappa_{\mathrm{Rx}}=\kappa$, then

$$
\kappa^{\star}(\mu)=\left\lceil\frac{2 \mu}{1-4 \mu}\right\rceil, \quad 0 \leq \mu<\frac{1}{4} .
$$

Proof: Follows from Theorem 2 and simple algebraic manipulations.

In particular, one round of conferencing, $\kappa_{\mathrm{Tx}}=\kappa_{\mathrm{Rx}}=1$, suffices when both transmitter- and receiver-conferencing prelogs are below $1 / 6$. Two rounds of conferencing, $\kappa_{\mathrm{Tx}}=\kappa_{\mathrm{Rx}}=2$, suffice when the conferencing prelogs are below $1 / 5$, and generally $\kappa_{\mathrm{Tx}}=\kappa_{\mathrm{Rx}}=\kappa$ conferencing rounds suffice when the conferencing prelogs $\mu_{\mathrm{Tx}}=\mu_{\mathrm{Rx}}=\mu$ satisfy

$$
\mu \leq \frac{\kappa}{2+4 \kappa}
$$

We terminate this section by focusing on the scenario with only transmitter-conferencing $\left(\mu_{\mathrm{Rx}}=0\right)$ or only receiverconrerencing $\left(\mu_{\mathrm{Tx}}=0\right)$. In these two cases, the expression for $\mathbf{S}(\boldsymbol{\kappa}, \boldsymbol{\mu})$ simplifies as follows:

Corollary 3 (Transmitter conferencing Only or Receiver Conferencing Only):
If $\mu_{\mathrm{Tx}}=0$, then

$$
\mathrm{S}(\boldsymbol{\kappa}, \boldsymbol{\mu})= \begin{cases}\frac{1+2 \mu_{\mathrm{Rx}}}{2}, & 2 \mu_{\mathrm{Rx}}+2 \pi_{\mathrm{Rx}} \leq 1 \\ \frac{2 \kappa_{\mathrm{Rx}}+1}{2 \kappa_{\mathrm{Rx}}+2}, & \text { otherwise }\end{cases}
$$

for all $\kappa \in \mathbb{Z}^{+} \times \mathbb{Z}^{+}$and $\mu_{\mathrm{Rx}} \in[0, \infty)$.

- If $\mu_{\mathrm{Rx}}=0$, then

$$
\mathrm{S}(\boldsymbol{\kappa}, \boldsymbol{\mu})= \begin{cases}\frac{1+2 \mu_{\mathrm{Tx}}}{2}, & 2 \mu_{\mathrm{Tx}}+2 \pi_{\mathrm{Tx}} \leq 1 \\ \frac{2 \kappa_{\mathrm{Tx}}+1}{2 \kappa_{\mathrm{Tx}}+2}, & \text { otherwise. }\end{cases}
$$

for all $\kappa \in \mathbb{Z}^{+} \times \mathbb{Z}^{+}$and $\mu_{\mathrm{Tx}} \in[0, \infty)$.

We now determine the minimum number of conferencing rounds required to achieve the same per-user MG as with an unlimited number of rounds when there is only transmitter-conferencing. (Similar results hold also for only receiver-conferencing only.) For $\mu \in[0,1 / 2]$, let

$$
\kappa_{\mathrm{Tx}}^{\star}(\mu):=\min _{\kappa}\left\{\kappa \in \mathbb{Z}: \mathbf{S}(\kappa, 0, \mu, 0)=\mathbf{S}_{\infty}(\mu, 0)\right\} .
$$

Proposition 4 (Sufficient Conferencing Rounds With Transmitter Conferencing Only): If $\mu_{\mathrm{Rx}}=0$, then

$$
\kappa_{\mathrm{Tx}}^{\star}(\mu)=\left\lceil\frac{2 \mu}{1-2 \mu}\right\rceil, \quad 0 \leq \mu<\frac{1}{2} .
$$

Proof: Follows from Corollary 3.

\section{Proof of Achievability of Theorem 2}

\section{A. Overview}

We now present a coding strategy that achieves $S^{\star}(\boldsymbol{\kappa}, \boldsymbol{\mu})$. The strategy will time-share between one, two or three different coding schemes - depending on the particular values of $\boldsymbol{\kappa}$ and $\boldsymbol{\mu}$. To this end, let us divide the blocklength $n$ in three consecutive periods of lengths $N_{1}, N_{2}$ and $N_{3}$ channel symbols, as shown in Fig. 11, so that $n=N_{1}+N_{2}+N_{3}$. Here $n$ and, therefore, $N_{1}, N_{2}$ and $N_{3}$ can be chosen arbitrarily large.

- Period 1: During period 1 (the first $N_{1}$ channel uses) we will use a scheme that employs both Tx- and Rxconferencing, assuming that the prelogs $\mu_{\mathrm{Tx}}$ and $\mu_{\mathrm{Rx}}$ are both positive. If $\mu_{\mathrm{Tx}}=0$ or $\mu_{\mathrm{Rx}}=0$, then we will remove period 1 by setting $N_{1}=0$.

- Period 2: During period 2 (channel uses $N_{1}+1$ to $\left.N_{1}+N_{2}\right)$ we will use a scheme that employs either Tx-conferencing (when $\pi_{\mathrm{Rx}}<\pi_{\mathrm{Tx}}$ ) or Rx-conferencing 


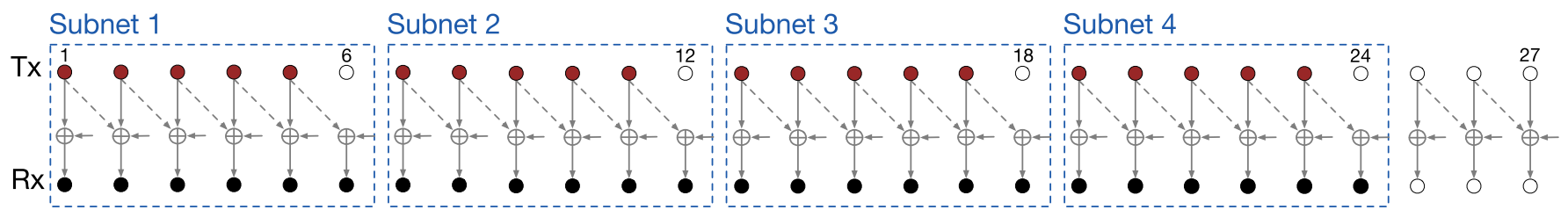

Fig. 12. Transmitter and receiver conferencing scheme: The network is decomposed into $\gamma$ non-interfering subnets, where each subnet consists of $(\beta-1)$ active transmitters and $\beta$ active receivers. Red nodes represent active transmitters, black nodes represent active receivers, and white nodes represent deactivated transmitters. Example parameters: $K=27, \imath=1, \beta=6$ and $\gamma=4$.

(when $\pi_{\mathrm{Tx}}>\pi_{\mathrm{Rx}}$ ), but not both. If $\pi_{\mathrm{Rx}}=\pi_{\mathrm{Tx}}$, then we will remove period 2 by setting $N_{2}=0$.

- Period 3: During period 3 (the last $N_{3}$ channel uses) we will use a scheme that does not employ Tx- or Rx-conferencing.

To simplify exposition, suppose that

$$
\pi_{\mathrm{Tx}} \geq \pi_{\mathrm{Rx}}
$$

so that we only use Tx-conferencing during period 2. (The case $\pi_{\mathrm{Tx}}<\pi_{\mathrm{Rx}}$ can be treated by exchanging the subscripts $\mathrm{Tx}$ and Rx everywhere in the following arguments.) Choose

$$
\begin{aligned}
& N_{1}:=\min \left\{n, n \pi_{\mathrm{Rx}}\left(2 \kappa_{\mathrm{Tx}}+2 \kappa_{\mathrm{Rx}}+2\right)\right\} \\
& N_{2}:=\min \left\{n-N_{1}, n\left(\pi_{\mathrm{Tx}}-\pi_{\mathrm{Rx}}\right)\left(2 \kappa_{\mathrm{Tx}}+2\right)\right\} \\
& N_{3}:=n-N_{1}-N_{2} .
\end{aligned}
$$

The length $N_{1}$ is chosen as large as possible until our Period-1 scheme exhausts one of the two conferencing-prelogs $\mu_{\mathrm{Tx}}$ or $\mu_{\mathrm{Rx}}$. Because of assumption (22), the scheme exhausts the receiver-conferencing prelog $\mu_{\mathrm{Rx}}$ in this Period 1 , and therefore in Period 2 we use a scheme with only transmitterconferencing. The length $N_{2}$ is chosen as large as possible until our Period-2 scheme exhausts also the transmitterconferencing prelog.

We detail the coding schemes used in each of the three periods.

Period 1: We will timeshare between $2 \kappa_{\mathrm{Tx}}+2 \kappa_{\mathrm{Rx}}+2$ instances of the coding scheme described in Section V-B but where we make sure that each (sub)message is sent at a rate not exceeding $(1 / 2) \log (1+P) .^{2}$ Each of these instances is used over an equally long interval, i.e., over $\left\lfloor N_{1} /\left(2 \kappa_{\mathrm{Tx}}+\right.\right.$ $\left.\left.2 \kappa_{\mathrm{Rx}}+2\right)\right\rfloor$ channel symbols, and employs a different value of the parameter $\imath \in\left\{1, \ldots, 2 \kappa_{\mathrm{Tx}}+2 \kappa_{\mathrm{Rx}}+2\right\}$. Varying the parameter $l$ over $1, \ldots, 2 \kappa_{\mathrm{Tx}}+2 \kappa_{\mathrm{Rx}}+2$ varies the utilised resources (transmit powers and conferencing links) as well as the served $\mathrm{Tx} / \mathrm{Rx}$ pairs in a round robin manner.

Period 2: We will timeshare between $2 \kappa_{\mathrm{Tx}}+2$ instances of the coding scheme in Section $\mathrm{V}-\mathrm{C}$, where we again make sure that each message is sent at a rate not exceeding $(1 / 2)$ $\log (1+P) .^{3}$ Each of these instances is used over an equally-long interval, i.e., over $\left\lfloor N_{2} /\left(2 \kappa_{\mathrm{Tx}}+2\right)\right\rfloor$ channel uses, and employs a different value for the parameter $\imath=1, \ldots, 2 \kappa_{\mathrm{Tx}}+2$.

\footnotetext{
${ }^{2}$ This ensures that also each Rx-conferencing link is used at a rate of at most $(1 / 2) \log (1+P)$.

${ }^{3}$ This again ensures that each Rx-conferencing link is used at a rate of at most $(1 / 2) \log (1+P)$.
}

Period 3: We will employ the scheme in Section V-D. No conferencing is used.

We now present the coding schemes that we time-share in the various transmission periods.

\section{B. Tx- and Rx-Conferencing in Period 1}

Let

$$
\beta:=2 \kappa_{\mathrm{Tx}}+2 \kappa_{\mathrm{Rx}}+2 .
$$

Choose $l \in\{1, \ldots, \beta\}$ arbitrarily, and define

$$
\gamma:=\left\lfloor\frac{K-\imath+1}{\beta}\right\rfloor .
$$

1) Split the Network Into $\gamma$ Subnetworks: We first split the network into $\gamma$ identical subnets that do not interfere each other. To this end, we will deactivate (silence) every transmitter ${ }^{4}$ with an index

$$
\begin{aligned}
& k \in \mathcal{S} \\
& :=\{1, \ldots, l-1\} \cup\{l+\beta-1, l+2 \beta-1, \ldots, l+\gamma \beta-1\} \\
& \cup\{l+\gamma \beta, \ldots, K\} .
\end{aligned}
$$

That means, every silenced Tx $k \in \mathcal{S}$ sets its channel inputs $X_{k}^{n}$ deterministically to 0 . Moreover, every such silenced Tx $k \in$ $\mathcal{S}$ can send and receive conferencing messages only to and from its left-neighbour Tx $k-1$, but not its right-neighbour Tx $k+1$. Similarly, each corresponding $\operatorname{Rx} k \in \mathcal{S}$ can send and receive conferencing messages only to and from its leftneighbour $\mathrm{Rx} k-1$, but not its right-neighbour $\mathrm{Rx} k+1$. According to these assumptions, the various subnets do not interfere and they each consist of $(\beta-1)$ active transmitters and $\beta$ active receivers - an example is illustrated in Figure 12.

2) Communication Within a Subnet: Since the subnets are identical and do not interfere with one another, we need only describe the coding scheme for subnet 1 (transmitters $l, \ldots, l+$ $\beta-2$ and receives $l, \ldots, l+\beta-1$ ), where we communicate source messages

$$
\left(M_{l}, M_{l+1}, \ldots, M_{l+\beta-1}\right) .
$$

Source message $M_{l+\beta-\kappa_{\mathrm{Rx}}-1}$ will be handled in a special way: We will split it into independent sub-messages

$$
M_{l+\beta-\kappa_{\mathrm{Rx}}-1}=\left(M_{l+\beta-\kappa_{\mathrm{Rx}}-1}^{\mathrm{Tx}}, M_{l+\beta-\kappa_{\mathrm{Rx}}-1}^{\mathrm{Rx}}\right),
$$

\footnotetext{
${ }^{4}$ Transmitters $1, \ldots, l-1$ and $l+\gamma \beta, \ldots, K$ have been deactivated to simplify the following presentation. In fact, we could improve the scheme's performance by, for example, reactivating transmitters $1,3,5, \ldots, i-1$ (each reactivated transmitter can communicate with its receiver over an interferencefree Gaussian point-to-point channel). Such reactivations, however, will not improve the scheme's asymptotic $(K \rightarrow \infty)$ per-user MG.
} 


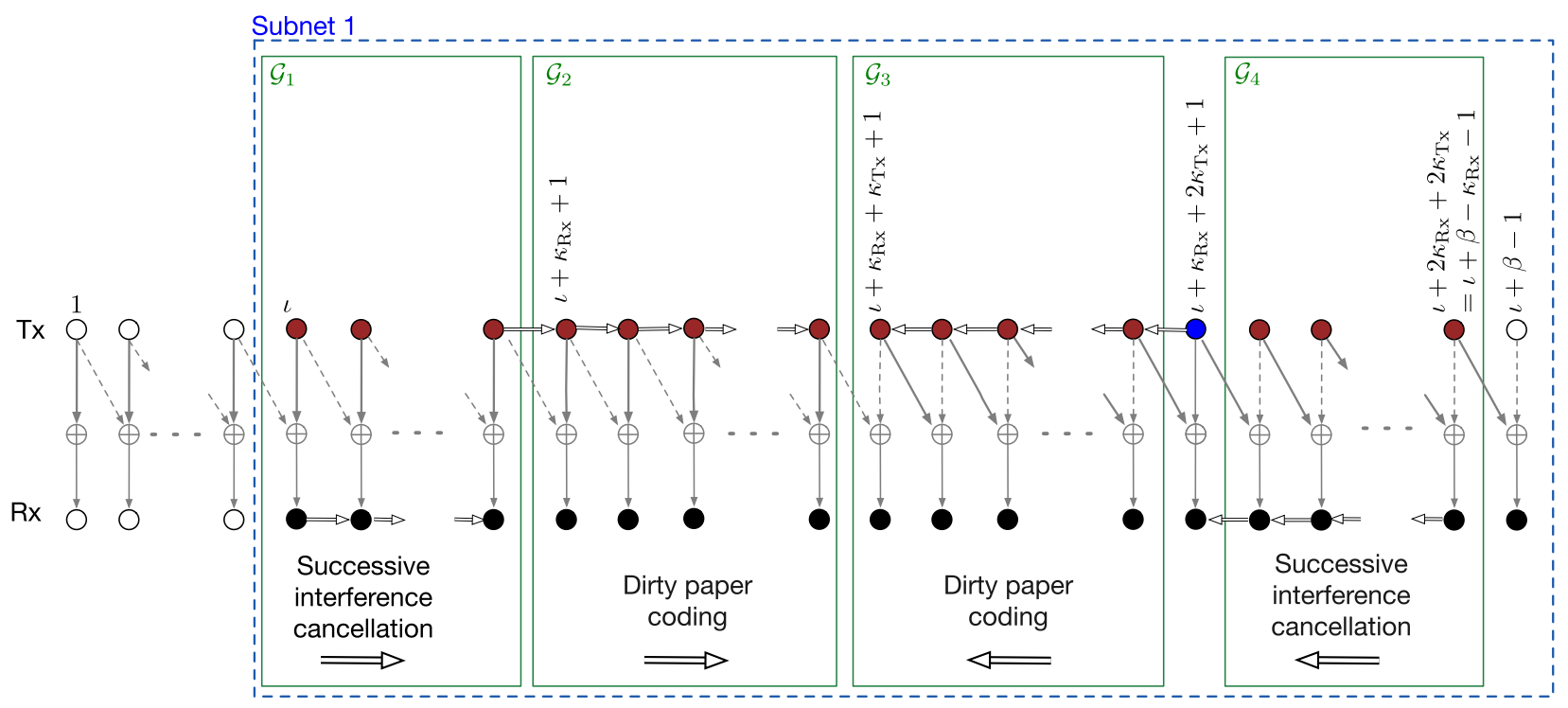

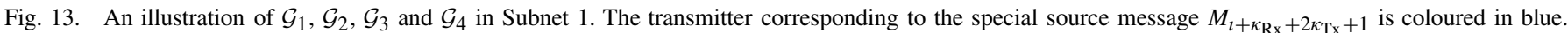
The figure also shows the Tx- and Rx-conferencing links that are effectively used in our scheme, and it indicates that the desired communication paths for group 1 and 2 messages are the direct links, and for group 3 and 4 messages the diagonal links.

where $M_{l+\beta-\kappa_{\mathrm{Rx}}-1}^{\mathrm{Tx}}$ has rate $R_{t+\beta-\kappa_{\mathrm{Rx}}-1}^{\mathrm{Tx}}$ and $M_{l+\beta-\kappa_{\mathrm{Rx}}-1}^{\mathrm{Rx}}$ has rate $R_{l+\beta-\kappa_{\mathrm{Rx}}-1}^{\mathrm{Rx}}$. We will partition the remaining source messages in (24) into four groups $\mathcal{G}_{1}, \mathcal{G}_{2}, \mathcal{G}_{3}$, and $\mathcal{G}_{4}$, see Figure 13. In our scheme, the messages in each group are transmitted using a different strategy. The special source message $M_{l+\beta-\kappa_{\mathrm{Rx}}-1}^{\mathrm{Tx}}$ is transmitted in the same way as source messages in $\mathcal{G}_{3}$, and special source message $M_{l+\beta-\kappa_{\mathrm{Rx}}-1}^{\mathrm{Rx}}$ is transmitted in the same way as source messages in $\mathcal{G}_{4}$.

The messages in each of the four groups $\mathcal{G}_{1}, \ldots, \mathcal{G}_{4}$ are transmitted using a different scheme. The transmission of messages in group $\mathcal{G}_{1}$ follows the data-sharing approach of Section III-B, and only uses left-to-right receiverconferencing. The group contains $\kappa_{\mathrm{Rx}}+1$ messages, which corresponds to the largest number of successive transmitter/receiver pairs that can communicate at prelog 1 using this data-sharing approach, see Section III-B. The transmission of messages in group $\mathcal{G}_{2}$ follows the compression-approach of Section III-A, and only uses left-to-right transmitterconferencing. The group contains $\kappa_{\mathrm{Tx}}$ messages. This is the largest number of successive transmitter/receiver pairs that can communicate at prelog 1 with our proposed scheme. (Notice that in Section III-A $\kappa_{\mathrm{Tx}}+1$ transmitter/receiver pairs could communicate at prelog 1 with the compressionapproach, however there, we started with an interferencefree transmitter/receiver-pair on the left). Messages in groups $\mathcal{G}_{3}$ are transmitted using a compression-approach but now for right-to-left transmitter-conferencing. Again, the set $\mathcal{G}_{3}$ is chosen as large as possible so that each of the messages can be communicated at prelog 1 . Finally, messages in group $\mathcal{G}_{4}$ are transmitted using a data-sharing approach but now for rightto-left receiver-conferencing. As for the other groups, each of the messages in this group is communicated at prelog 1 .

We next describe how to partition the messages into groups $\mathcal{G}_{1}, \mathcal{G}_{2}, \mathcal{G}_{3}$ and $\mathcal{G}_{4}$, and we sketch how to communicate the source messages in these groups. For more technical details on these schemes see Appendix A.

3) Group 1 (Successive Interference Cancellation From Left to Right, See Figure 14): The first group of source messages,

$$
\left\{M_{k}: k \in \mathcal{G}_{1}\right\}
$$

with

$$
\mathcal{G}_{1}:=\left\{l, l+1, \ldots l+\kappa_{\mathrm{Rx}}\right\},
$$

is communicated using point-to-point channel codes with successive interference cancellation from left-to-right at the receivers. Specifically, each Tx $k \in \mathcal{G}_{1}$ uses a Gaussian pointto-point code of power $P$ to transmit its source message $M_{k}$. Inputs $X_{k}^{n}\left(M_{k}\right)$ thus only depend on $M_{k}$.

The decoding procedure is depicted in Figure 14 and described with more technical details in Appendix A-A.

Recall that we deactivated Tx $(l-1)$ (the last transmitter in the previous subnet). Rx $l$ (the first receiver in group 1) thus observes channel outputs

$$
Y_{l}^{n}=X_{l}^{n}\left(M_{l}\right)+Z_{l}^{n},
$$

based on which it decodes its desired source message $M_{l}$.

Rx $l$ also describes its decoded source message $\hat{M}_{l}$ over the conferencing link to $\mathrm{Rx}(l+1)$ (its immediate right-neighbour).

$\mathrm{Rx}(l+1)$ uses this conferencing message to reconstruct $\alpha_{l+1} X_{l}^{n}\left(\hat{M}_{l}\right)$. It then forms

$$
\hat{Y}_{l+1}^{n}=Y_{l+1}^{n}-\alpha_{l+1} X_{l}^{n}\left(\hat{M}_{l}\right)
$$

and decodes source message $M_{l+1}$ based on this difference. $\operatorname{Rx}(l+1)$ also describes $\hat{M}_{l+1}$ over the conferencing link to $\mathrm{Rx}(l+2)$ (its immediate right-neighbour).

Notice that whenever $\hat{M}_{l}=M_{l}, \operatorname{Rx}(l+1)$ decodes $M_{l+1}$ based on the interference-free signal

$$
\hat{Y}_{l+1}^{n}=X_{l+1}^{n}\left(M_{l+1}\right)+Z_{l+1}^{n} .
$$




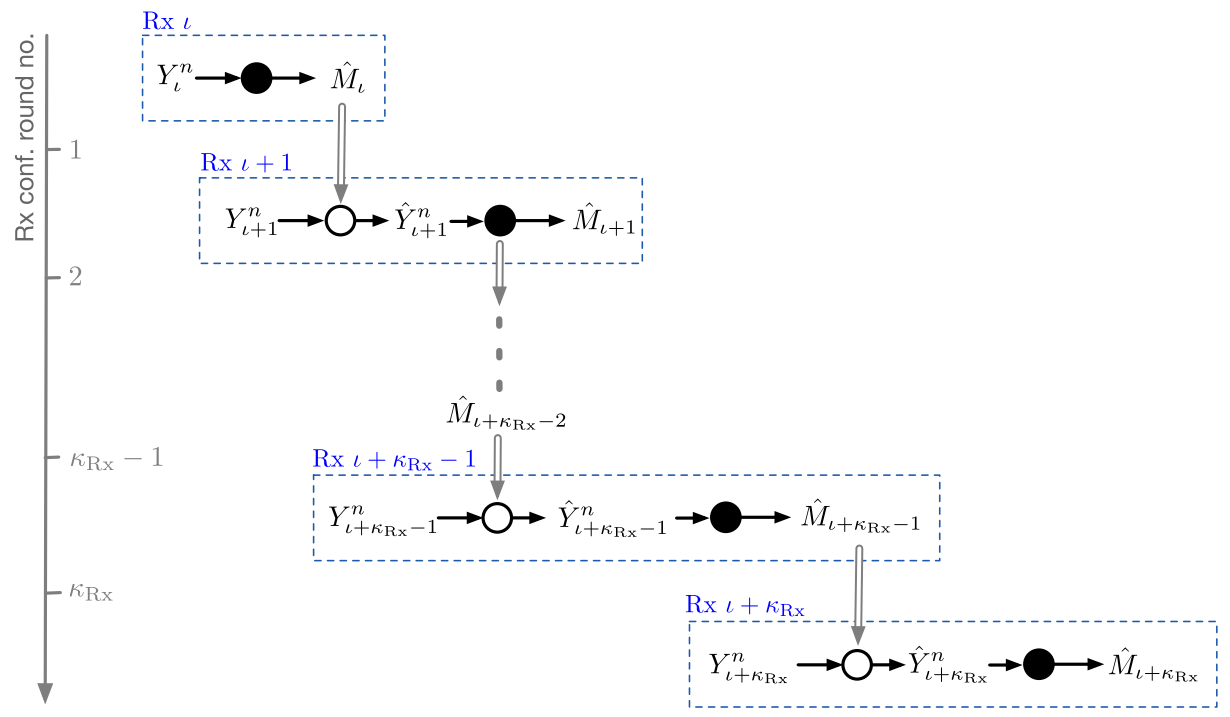

Fig. 14. Group 1: Left-to-right successive cancellation at the receivers. The white circles represent interference cancellation and the black circles represent channel decoding. Top-down arrows represent communications over left-to-right Tx-conferencing links.

The same procedure is continued for receivers $k=l+$ $2, \ldots, l+\kappa_{\mathrm{Rx}}$. Specifically, each of these receivers performs the following three steps:

1) It reconstructs interference $\alpha_{k} X_{k-1}^{n}\left(\hat{M}_{k-1}\right)$ using the conferencing message $\hat{M}_{k-1}$ obtained from Rx $k-1$.

2) It forms the presumingly interference-free signal

$$
\hat{Y}_{k}^{n}=Y_{k}^{n}-\alpha_{k} \hat{X}_{k-1}^{n}\left(\hat{M}_{k-1}\right),
$$

and it decodes source message $M_{k}$ based on this difference.

3) It sends the decoded source message $\hat{M}_{k}$ over the conferencing link to $\mathrm{Rx}(k+1)$. The last receiver, $\mathrm{Rx}\left(l+\kappa_{\mathrm{Rx}}\right)$, does not send anything over the conferencing link (it skips this third step).

Notice that the described scheme requires only left-toright $\mathrm{Rx}$-conferencing; neither Tx-conferencing nor right-toleft conferencing are needed. Also, $\mathrm{Rx}(l+j-1)$ (the $j$-th receiver of group $\left.\mathcal{G}_{1}\right)$, for $j \in\left\{1, \ldots, \kappa_{\mathrm{Rx}}-1\right\}$, has to wait until it obtains the Rx-conferencing message from its leftneighbour before it can start performing above three steps. It can thus send its own conferencing message $\hat{M}_{l+j-1}$ only in Rx-conferencing round $j$.

Finally, we notice that for each $k$, if $\operatorname{Rx} k-1$ has correctly decoded its message, i.e., $\hat{M}_{k-1}=M_{k-1}$, then Rx $k$ decodes the source message $M_{k}$ based on the interference-free signal $\hat{Y}_{k}^{n}=X_{k}^{n}+Z_{k}^{n}$. Source messages $M_{l}, \ldots, M_{l+\kappa_{\mathrm{Rx}}}$ can thus be decoded with vanishingly small probability of error as $n \rightarrow$ $\infty$, whenever (see also Lemma 2 in Appendix A-A)

$$
R_{k}<\frac{1}{2} \log (1+P), \quad \forall k \in \mathcal{G}_{1}
$$

and

$$
R_{\mathrm{Rx}}>R_{k}, \quad \forall k \in \mathcal{G}_{1} .
$$

4) Group 2 (Dirty-Paper Coding From Left to Right, See Figure 15): The second group of source messages,

$$
\left\{M_{k}: k \in \mathcal{G}_{2}\right\}
$$

with

$$
\mathcal{G}_{2}:=\left\{l+\kappa_{\mathrm{Rx}}+1, l+\kappa_{\mathrm{Rx}}+2, \ldots, l+\kappa_{\mathrm{Rx}}+\kappa_{\mathrm{Tx}}\right\},
$$

will be communicated using dirty-paper coding to mitigate the interference from the left. The encoding procedure is depicted in Figure 15 and is explained with more technical details in Appendix A-B.

To facilitate dirty-paper coding at $\mathrm{Tx}\left(l+\kappa_{\mathrm{Rx}}+1\right)$ (the first transmitter in group 2), Tx $\left(l+\kappa_{\mathrm{Rx}}\right)$ (the last transmitter in group 1) quantises its inputs signal $X_{l+\kappa_{\mathrm{Rx}}}^{n}$ using a rate $(1 / 2) \log (1+P)$ quantiser and sends the resulting quantisation message to Tx $\left(l+\kappa_{\mathrm{Rx}}+1\right)$.

Upon receiving the quantisation message, $\mathrm{Tx}\left(l+\kappa_{\mathrm{Rx}}+1\right)$ reconstructs the quantised inputs $\hat{X}_{l+\kappa_{\mathrm{Rx}}}^{n}$ and encodes its source message $M_{k}$ using a power $P$ dirty-paper code that eliminates the interference $\alpha_{l+\kappa_{\mathrm{Rx}}+1} \hat{X}_{l+\kappa_{\mathrm{Rx}}}^{n}$.

Tx $\left(l+\kappa_{\mathrm{Rx}}+1\right)$ also quantises its produced inputs $X_{l+\kappa_{\mathrm{Rx}}+1}^{n}$ using a rate $(1 / 2) \log (1+P)$ quantiser, and sends the quantisation message to Tx $\left(l+\kappa_{\mathrm{Rx}}+2\right)$ (its right-neighbour).

$\operatorname{Rx}\left(l+\kappa_{\mathrm{Rx}}+1\right)$ decodes source message $M_{l+\kappa_{\mathrm{Rx}}+1}$ by applying dirty-paper decoding to its outputs

$$
Y_{l+\kappa_{\mathrm{Rx}}+1}^{n}=\alpha_{l+\kappa_{\mathrm{Rx}}+1} X_{l+\kappa_{\mathrm{Rx}}}^{n}+X_{l+\kappa_{\mathrm{Rx}}+1}^{n}+Z_{l+\kappa_{\mathrm{Rx}}+1}^{n} .
$$

Notice that

$$
\alpha_{l+\kappa_{\mathrm{Rx}}+1} \hat{X}_{l+\kappa_{\mathrm{Rx}}}^{n}-\alpha_{l+\kappa_{\mathrm{Rx}}+1} X_{l+\kappa_{\mathrm{Rx}}}^{n}
$$

has variance close to

$$
n \alpha_{l+\kappa_{\mathrm{Rx}}+1}^{2} \frac{P}{P+1}
$$

and thus for $P \gg 1$ the dirty-paper code precancels the predominant part of the interfering signal $\alpha_{l+\kappa_{\mathrm{Rx}}+1} X_{l+\kappa_{\mathrm{Rx}}}^{n}$. 




Fig. 15. Group 2: Transmitter dirty-paper coding from left to right. The dark circles represent dirty-paper channel encoders, and the white circles represent vector Gaussian quantisers. Top-down arrows represent communications of quantised input signals over the Tx-conferencing links to right-neighbours.

The procedure is repeated for Tx/Rxs $k=\imath+\kappa_{\mathrm{Rx}}+2, \ldots, l+$ $\kappa_{\mathrm{Rx}}+\kappa_{\mathrm{Tx}}$. Each such Tx $k$ performs the following three steps:

1) Using the conferencing message from $\mathrm{Tx}(k-1)$, it reconstructs the quantised signal $\hat{X}_{k-1}^{n}$.

2) It encodes and transmits its source message $M_{k}$ using a power- $P$ dirty-paper code that eliminates interference $\alpha_{k} \hat{X}_{k-1}^{n}$.

3) It quantises its input signals $X_{k}^{n}$ with a rate $(1 / 2) \log (1+$ $P)$ quantiser and sends the produced quantisation message over the conferencing link to Tx $k+1$.

$\mathrm{Tx}\left(l+\kappa_{\mathrm{Rx}}+\kappa_{\mathrm{Tx}}\right)$ (the last transmitter in group $\left.\mathcal{G}_{2}\right)$ sends no conferencing message; it skips step 3.

Finally, each one of the corresponding $\mathrm{Rx} k$ decodes its desired source message $M_{k}$ using dirty-paper decoding based on its outputs $Y_{k}^{n}$.

Notice that the described scheme requires only left-to-right Tx-conferencing; neither right-to-left Tx-conferencing nor $\mathrm{Rx}$-conferencing are needed. Also, $\mathrm{Tx}\left(l+\kappa_{\mathrm{Rx}}+j\right)$, (the $j$-th transmitter of group $\mathcal{G}_{2}$ ), for $j \in\left\{1, \ldots, \kappa_{\mathrm{Tx}}-1\right\}$, has to wait until it obtains the Tx-conferencing message from its leftneighbour before it can perform above three steps. It can thus send its own Tx-conferencing message to its right-neighbour only in Tx-conferencing round $j+1$.

Finally, as we show in Appendix A-B, through a careful design of the vector-quantisers and the dirty-paper codes and when $P \gg 1$, the predominant part of the interference $\alpha_{k} X_{k-1}^{n}$ experienced at $\mathrm{Rx} k$ can be precanceled. As a consequence, source messages $M_{l+\kappa_{\mathrm{Rx}}+1}, \ldots, M_{l+\kappa_{\mathrm{Rx}}+\kappa_{\mathrm{Tx}}}$ can be decoded with vanishingly small probability of error as $n \rightarrow \infty$, whenever (see also Lemma 3 in Appendix A-B)

$$
R_{k}<\frac{1}{2} \log \left(1+\frac{P}{1+\alpha_{k}^{2} \frac{P}{P+1}}\right), \quad \forall k \in \mathcal{G}_{2}
$$

and

$$
R_{\mathrm{Tx}}>\frac{1}{2} \log (1+P) .
$$

Source messages in groups 1 and 2 were transmitted over the "direct" links from a Tx $k$ to its corresponding $\mathrm{Rx} k$. The "diagonal" links from a Tx $k$ to its right-neighbouring $\mathrm{Rx}(k+1)$ only carried interference that had to be mitigated. In contrast, source messages in groups 3 and 4 are transmitted over the "diagonal" links, whereas the "direct" links carry interference that has to be mitigated. Without conferencing, there exists no desired communication path over the diagonal links, since the source message desired by $\mathrm{Rx} k$ is a priori unknown at its left-neighbour Tx $(k-1)$. Setting up such a path requires concatenating the diagonal link from Tx $(k-1)$ to $\mathrm{Rx} k$ with a preceding right-to-left Tx-conferencing from Tx $k$ to $\mathrm{Tx}(k-1)$ or with a subsequent right-to-left $\mathrm{Rx}$-conferencing from $\mathrm{Rx} k$ to $\mathrm{Rx}(k-1)$, see (34) and (32) ahead.

We now describe transmission of messages in group 4, followed by transmission of messages in group 3.

5) Group 4 (Successive Interference Cancellation From Right to Left): The fourth group of messages,

$$
\left\{M_{l+\beta-\kappa_{\mathrm{Rx}}-1}^{\mathrm{Rx}}\right\} \cup\left\{M_{k}: k \in \mathcal{G}_{4}\right\}
$$

with

$$
\mathcal{G}_{4}:=\left\{l+\beta-\kappa_{\mathrm{Rx}}, \ldots, l+\beta-2\right\},
$$

is communicated using point-to-point channel codes with right-to-left successive interference cancellation at the decoders. (See Appendix A-C for more technical details.) 
Specifically, each source message $M_{k}{ }^{5}$ is sent over the following communication path:

$$
\mathrm{Tx} k \longrightarrow \mathrm{Rx}(k+1) \longrightarrow \mathrm{Rx} k .
$$

Each Tx $k \in \mathcal{G}_{4}$ uses a Gaussian point-to-point code of power $P$ to transmit its source message $M_{k}$.

$\operatorname{Rx} l+\beta-1$ (the last receiver in the subnet) observes channel outputs

$$
Y_{l+\beta-1}^{n}=\alpha_{l+\beta-1} X_{l+\beta-2}^{n}\left(M_{l+\beta-2}\right)+Z_{l+\beta-1}^{n},
$$

because we silenced its corresponding $\operatorname{Tx}(l+\beta-1)$. It decodes source message $M_{l+\beta-2}$ from these channel outputs and describes the decoded message $\hat{M}_{l+\beta-2}$ over the conferencing link to $\operatorname{Rx}(l+\beta-2)$ (its immediate left-neighbour).

$\operatorname{Rx}(l+\beta-2)$ declares the obtained conferencing message $\hat{M}_{l+\beta-2}$ as its guess of $M_{l+\beta-2}$. It further uses the conferencing message to reconstruct $X_{l+\beta-2}^{n}\left(\hat{M}_{l+\beta-2}\right)$ and forms

$$
\hat{Y}_{l+\beta-2}^{n}=Y_{l+\beta-2}^{n}-X_{l+\beta-2}^{n}\left(\hat{M}_{l+\beta-2}\right) .
$$

$\operatorname{Rx}(l+\beta-2)$ finally decodes source message $M_{l+\beta-3}$ from this difference and describes $\hat{M}_{l+\beta-3}$ over the conferencing link to $\operatorname{Rx}(\imath+\beta-3)$.

Whenever $\hat{M}_{l+\beta-2}=M_{l+\beta-2}$,

$$
\hat{Y}_{l+\beta-2}^{n}=\alpha_{l+\beta-2} X_{l+\beta-3}^{n}\left(M_{l+\beta-3}\right)+Z_{l+\beta-2}^{n},
$$

and Rx $(l+\beta-2)$ decodes source message $M_{l+\beta-3}$ based on an interference-free signal.

The described procedure is repeated for receivers $k=\imath+$ $\beta-3, \ldots, l+\beta-\kappa_{\mathrm{Rx}}-1$ in decreasing order. Specifically, each such $\mathrm{Rx} k$ performs four steps:

1) Using the conferencing message $\hat{M}_{k}$ from $\mathrm{Rx}(k+1)$, it reconstructs the "interference" $X_{k}^{n}\left(\hat{M}_{k}\right)$.

2) It forms $\hat{Y}_{k}^{n}:=Y_{k}^{n}-X_{k}^{n}\left(\hat{M}_{k}\right)$ and decodes source message $M_{k-1}$ based on this difference.

3) It sends the decoded source message $\hat{M}_{k-1}$ over the conferencing link to $\mathrm{Rx}(k-1)$.

4) It declares $\hat{M}_{k}$ as its guess of source message $M_{k}$.

Notice that the described scheme requires only right-toleft Rx-conferencing; neither left-to-right Rx-conferencing nor Tx-conferencing are needed. $\operatorname{Rx}(l+\beta-1)$ (the last receiver in the subnet) sends its conferencing message $\hat{M}_{l+\beta-2}$ in the first Rx-conferencing round. For each $j \in\left\{2, \ldots, \kappa_{\mathrm{Rx}}\right\}$, $\operatorname{Rx}(l+\beta-j)$ (the $j$-th right-most receiver in the subnet) has to wait until it obtains the conferencing message from its right-neighbour before it can start performing above four steps. It can thus send its own conferencing message $\hat{M}_{l+\beta-j-1}$ only in Rx-conferencing round $j$.

Finally, we notice that if $\mathrm{Rx}(k+2)$ 's decoding was successful, i.e., $\hat{M}_{k+1}=M_{k+1}$, then $\hat{X}_{k+1}^{n}=X_{k+1}^{n}$ and $\hat{Y}_{k+1}^{n}=\alpha_{k+1} X_{k}^{n}+Z_{k+1}^{n}$, and $\mathrm{Rx}(k+1)$ can thus decode source message $M_{k}$ based on an interference-free signal.

Consequently, source messages $M_{l+\beta-\kappa_{\mathrm{Rx}}-1}^{\mathrm{Rx}}$, $M_{l+\beta-\kappa_{\mathrm{Rx}}}, \ldots, M_{l+\beta-2}$ can be decoded with vanishingly

\footnotetext{
${ }^{5}$ For ease of notation, in the following paragraph we write simply $M_{l+\beta-\kappa_{\mathrm{Rx}}-1}$ for $M_{l+\beta-\kappa_{\mathrm{Rx}}-1}^{\mathrm{Rx}}$
}

small probability of error as $n \rightarrow \infty$, whenever

$$
\begin{gathered}
R_{k}<\frac{1}{2} \log \left(1+\alpha_{k+1}^{2} P\right), \quad k \in \mathcal{G}_{4}, \\
R_{l+\beta-\kappa_{\mathrm{Rx}}-1}^{\mathrm{Rx}}<\frac{1}{2} \log \left(1+\alpha_{l+\beta-\kappa_{\mathrm{Rx}}}^{2} P\right),
\end{gathered}
$$

and

$$
R_{\mathrm{Rx}}>\max \left\{R_{l+\beta-\kappa_{\mathrm{Rx}}-1}^{\mathrm{Rx}}, R_{l+\beta-\kappa_{\mathrm{Rx}}}, \ldots, R_{l+\beta-2}\right\} .
$$

6) Group 3 (Dirty-Paper Coding From Right to Left): The third group of messages

$$
\left\{M_{k}: k \in \mathcal{G}_{3}\right\} \cup\left\{M_{l+\beta-\kappa_{\mathrm{Rx}}-1}^{\mathrm{Tx}}\right\}
$$

with

$$
\mathcal{G}_{3}:=\left\{l+\beta-\kappa_{\mathrm{Rx}}-\kappa_{\mathrm{Tx}}, \ldots, l+\beta-\kappa_{\mathrm{Rx}}-2\right\},
$$

uses dirty-paper coding to mitigate the interference from the right. The desired communication path of a message $M_{k}$ is

$$
\mathrm{Tx} k \longrightarrow \mathrm{Tx}(k-1) \longrightarrow \mathrm{Rx} k
$$

and thus involves Tx-conferencing from right-to-left. More specifically, Tx $k$ prepares a transmit signal $X_{k-1}^{n}$ that encodes message $M_{k}$. It then describes it over the conferencing link to Tx $(k-1)$, which will transmit this signal.

To reduce notational clutter, let us temporarily fix $k=\imath+$ $\beta-\kappa_{\mathrm{Rx}}-1$. In the previous subsection we described how Tx $k$ (the special transmitter of Figure 13), generated its input signal $X_{k}^{n}$ in function of $M_{k}^{\mathrm{Rx}}$. Tx $k$ now also encodes its second message $M_{k}^{\text {Tx }}$ using a dirty-paper code of power $\alpha_{k}^{2} P^{2} /(P+1)$ that mitigates its own input signal $X_{k}^{n}$. Denote the produced dirty-paper sequence by $\Psi_{\mathrm{DPC}, k}\left(M_{k}^{\mathrm{Rx}}\right)$. Tx $k$ quantises a scaled version of the dirty-paper sequence,

$$
\Phi_{k}^{n}:=\frac{P+1}{P} \Psi_{\mathrm{DPC}, k}\left(M_{k}^{\mathrm{Rx}}\right)
$$

using a rate $(1 / 2) \log (1+P)$ quantiser and sends the resulting quantisation message over the conferencing link to Tx $(k-1)$ (its left neighbour).

Tx $(k-1)$ reconstructs the quantised sequence $\hat{\Phi}_{k}^{n}$ and transmits

$$
X_{k-1}^{n}=\alpha_{k}^{-1} \hat{\Phi}_{k}^{n}
$$

over the interference network. Rx $k$ decodes source message $M_{k}^{\mathrm{Tx}}$ by applying dirty-paper decoding to its outputs $Y_{k}^{n}$.

Notice that if there was no quantisation error,

$$
\Delta_{k}^{n}:=\hat{\Phi}_{k}^{n}-\Phi_{k}^{n}=0,
$$

then $\operatorname{Rx} k$ would observe outputs

$$
Y_{k}^{n}=\Psi_{\mathrm{DPC}, k}\left(M_{k}^{\mathrm{Rx}}\right)+X_{k^{n}}+Z_{k}^{n} .
$$

In this case, since the applied dirty-paper code precancels the "interference" $X_{k}^{n}$, source message $M_{k}$ could be transmitted with the same rates as over an interference-free channel.

Now, zero quantization error (35) is very unlikely. However, when the quantiser is chosen as in Appendix D, the normalized variance $\operatorname{Var}\left[(1 / n) \Delta_{k}^{n}\right]$ approaches $\alpha_{k}^{2} /(P+1)$ and is bounded in $P$. Treating the quantisation $\Delta_{k}^{n}$ simply as an additional 
noise, will thus not degrade the prelog rate of the source message $M_{k}$. (See Appendix A-D for a more detailed analysis.)

Let now $k=\imath+\beta-\kappa_{\mathrm{Rx}}-2$, one less than before, and consider transmitter Tx $k$. Tx $k$ encodes its own source message $M_{k}$ using a dirty-paper code of power $\alpha_{k}^{2} P^{2} /(P+$ 1) that mitigates its own input signal $X_{k}^{n}$. Let $\Psi_{\mathrm{DPC}, k}^{n}\left(M_{k}\right)$ denote the produced dirty-paper sequence. Tx $k$ applies a rate $(1 / 2) \log (1+P)$ quantiser to the scaled sequence

$$
\Phi_{k}^{n}:=\frac{P+1}{P} \Psi_{\mathrm{DPC}, k}^{n}\left(M_{k}\right)
$$

and sends the resulting quantisation message to Tx $(k-1)$.

This process is repeated for transmitters and receivers $k=\imath+\beta-\kappa_{\mathrm{Rx}}-3, \ldots, \imath+\beta-\kappa_{\mathrm{Rx}}-\kappa_{\mathrm{Tx}}$ in decreasing order. Each such Tx $k$ performs the following four steps ${ }^{6}$ :

1) Using the conferencing message from $\mathrm{Tx}(k+1)$, it reconstructs the quantised signal $\hat{\Phi}_{k+1}^{n}$.

2) It transmits $X_{k}^{n}=\alpha_{k+1}^{-1} \hat{\Phi}_{k+1}^{n}$ over the network.

3) It encodes source message $M_{k}$ using dirty-paper coding of power $\alpha_{k}^{2} P^{2} /(P+1)$ that mitigates its own inputs $X_{k}^{n}$. It then forms $\Phi_{k}^{n}=(P+1) / P \Psi_{\mathrm{DPC}, k}^{n}\left(M_{k}\right)$, where $\Psi_{\mathrm{DPC}, k}^{n}\left(M_{k}\right)$ denotes the produced dirty-paper sequence.

4) It quantises $\Phi_{k}^{n}$ using a rate $(1 / 2) \log (1+P)$ quantiser and sends the resulting quantisation bits over the conferencing link to Tx $(k-1)$.

Tx $\left(l+\kappa_{\mathrm{Rx}}+\kappa_{\mathrm{Tx}}+1\right)$ (the left-most transmitter only performs steps 1) and 4), but prepares and sends no conferencing message. Each receiver applies dirty-paper decoding to decode its desired message $M_{k}$ based on its outputs $Y_{k}^{n}$.

Notice that the described scheme requires right-to-left Tx-conferencing; neither left-to-right Tx-conferencing nor $\mathrm{Rx}$-conferencing are needed. Special Tx $\left(l+\kappa_{\mathrm{Rx}}+2 \kappa_{\mathrm{Tx}}+1\right)$ sends its conferencing message in Tx-conferencing round 1. Each Tx $\left(l+\beta-\kappa_{\mathrm{Rx}}-j\right)$, for $j \in\left\{2, \ldots, \kappa_{\mathrm{Tx}}-1\right\}$ can send its conferencing message only after receiving the conferencing message from its right-neighbour. Tx $\left(l+\beta-\kappa_{\mathrm{Rx}}-j\right)$ can thus send its conferencing message only in Tx-conferencing round $j+1$.

Finally, as we show in detail in Appendix A-D, through a careful design of the quantisers and the dirty-paper codes, source messages $M_{l+\beta-\kappa_{\mathrm{Rx}}-\kappa_{\mathrm{Tx}}}, \ldots, M_{l+\beta-\kappa_{\mathrm{Rx}}-1}^{\mathrm{Tx}}$ can be decoded with vanishingly small probability of error as $n \rightarrow \infty$ whenever the following holds:

$$
\begin{aligned}
R_{k} & <\frac{1}{2} \log \left(1+\frac{\alpha_{k}^{2} \frac{P^{2}}{P+1}}{1+\alpha_{k}^{2} \frac{P}{P+1}}\right), \quad k \in \mathcal{G}_{3}, \\
R_{l+\beta-\kappa_{\mathrm{Rx}}-1}^{\mathrm{Tx}} & <\frac{1}{2} \log \left(1+\frac{\frac{P^{2}}{P+1} \alpha_{l+\beta-\kappa_{\mathrm{Rx}}-1}^{2}}{1+\frac{P}{P+1} \alpha_{l+\beta-\kappa_{\mathrm{Rx}}-1}^{2}}\right)
\end{aligned}
$$

and

$$
R_{\mathrm{Tx}}>\frac{1}{2} \log (1+P) .
$$

\footnotetext{
${ }^{6}$ See Appendix A-D for more technical details.
}

\section{Tx-Conferencing in Period 2}

If we set $\kappa_{\mathrm{Rx}}=0$ everywhere in Subsection V-B, hence $\mathcal{G}_{1}=\mathcal{G}_{4}=\emptyset$, we obtain our scheme for period 2 in case of Tx-conferencing only. In this case, there is no need for a message $M_{l+\kappa_{\mathrm{Tx}}+2 \kappa_{\mathrm{Rx}}+1}^{\mathrm{Rx}}$ and we can set $M_{l+\kappa_{\mathrm{Tx}}+2 \kappa_{\mathrm{Rx}}+1}^{\mathrm{Tx}}=$

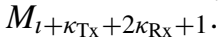

\section{No Conferencing in Period 3}

We silence all odd transmitters. This splits the network into a set of $\left\lceil\frac{K}{2}\right\rceil$ parallel Gaussian point-to-point channels. We use optimal point-to-point codes over these channels.

\section{E. Analysis}

1) Analysis of Period 1: As argued in (28), (30), (33) and (36) (see also Lemmas 2-5) in Appendix A, in each subnet we can transmit $\left(2 \kappa_{\mathrm{Tx}}+2 \kappa_{\mathrm{Tx}}+1\right)$-(sub)messages each of MG 1 . Thus, over the entire network which consists of $\gamma$ subnets, our scheme achieves a MG of

$$
\gamma\left(2 \kappa_{\mathrm{Tx}}+2 \kappa_{\mathrm{Rx}}+1\right)
$$

and a per-user MG of

$$
\mathrm{S}_{\text {period } 1}=\frac{2 \kappa_{\mathrm{Tx}}+2 \kappa_{\mathrm{Rx}}+1}{2 \kappa_{\mathrm{Tx}}+2 \kappa_{\mathrm{Rx}}+2} .
$$

We now analyse the communication over the conferencing links. We have for each subnet $g=0, \ldots, \gamma-1$ (see lemmas 2-5 in Appendix A):

- The $\kappa_{\mathrm{Tx}}$ consecutive transmitters $l+g \beta+\kappa_{\mathrm{Rx}}, \ldots, l+$ $g \beta+\kappa_{\mathrm{Rx}}+\kappa_{\mathrm{Tx}}-1$ send conferencing messages to their right-neighbours. Each of these messages corresponds to a decoded source message. Since we transmit all source messages at rates below $(1 / 2) \log (1+P)$, also the rates of the conferencing messages do not exceed $(1 / 2)$ $\log (1+P)$.

- The $\kappa_{\mathrm{Tx}}$ consecutive transmitters $l+(g+1) \beta-\kappa_{\mathrm{Rx}}-$ $\kappa_{\mathrm{Tx}}, \ldots, \imath+(g+1) \beta-\kappa_{\mathrm{Rx}}-1$ send conferencing messages to their left-neighbours. Each of these messages corresponds to a decoded source message. Since we transmit all source messages at rates below $(1 / 2) \log (1+P)$, also the rates of the conferencing messages do not exceed $(1 / 2) \log (1+P)$.

- The $\kappa_{\mathrm{Rx}}$ consecutive receivers $l+g \beta, \ldots, l+g \beta+$ $\kappa_{\mathrm{Rx}}-1$ send conferencing messages to their rightneighbours. Each of these messages corresponds to a rate $(1 / 2) \log (1+P)$ quantisation message. ${ }^{7}$

- Only the $\kappa_{\mathrm{Rx}}$ consecutive receivers $l+(g+1) \beta-$ $\kappa_{\mathrm{Rx}}, \ldots, l+(g+1) \beta-1$ send conferencing messages to their left-neighbours. Each of these messages corresponds to a rate $(1 / 2) \log (1+P)$ quantisation message.

Since $l$ varies from $1, \ldots, \beta$, we conclude that over the entire period each Tx-conferencing link is used for a

\footnotetext{
${ }^{7}$ More precisely, the rate should be slightly larger than $(1 / 2) \log (1+P)$. Through standard continuity considerations one can show that this does not change the set of achievable rates. It is thus a minor technicality, which we ignore.
} 
$\kappa_{\mathrm{Tx}} /\left(2 \kappa_{\mathrm{Tx}}+2 \kappa_{\mathrm{Rx}}+2\right)-$ th fraction of the time, and at a total rate not exceeding

$$
\mu_{\mathrm{Tx}, \text { Period } 1}=\frac{\kappa_{\mathrm{Tx}}}{2 \kappa_{\mathrm{Tx}}+2 \kappa_{\mathrm{Rx}}+2} \cdot \frac{1}{2} \log (1+P) .
$$

Similarly, each Rx-conferencing link is used for a $\kappa_{\mathrm{Rx}} /\left(2 \kappa_{\mathrm{Tx}}+\right.$ $\left.2 \kappa_{\mathrm{Rx}}+2\right)$-th fraction of the time, and at a total rate not exceeding

$$
\mu_{\mathrm{Rx}, \text { Period } 1}=\frac{\kappa_{\mathrm{Rx}}}{2 \kappa_{\mathrm{Tx}}+2 \kappa_{\mathrm{Rx}}+2} \cdot \frac{1}{2} \log (1+P) .
$$

2) Analysis of Period 2: Specializing the findings of the previous section to $\kappa_{\mathrm{Rx}}=0$, the per-user $\mathrm{MG}$ achieved in period 2 is

$$
\mathrm{S}_{\text {period } 2}=\frac{2 \kappa_{\mathrm{Tx}}+1}{2 \kappa_{\mathrm{Tx}}+2} .
$$

The Tx-conferencing links are used at rate

$$
\mu_{\mathrm{Tx}, \text { Period2 }}=\frac{\kappa_{\mathrm{Tx}}}{2 \kappa_{\mathrm{Tx}}+2} \cdot \frac{1}{2} \log (1+P),
$$

and the Rx-conferencing links are not used at all,

$$
\mu_{\mathrm{Rx}, \text { Period } 2}=0 \text {. }
$$

3) Analysis of Period 3: During the third period the achieved per-user MG is

$$
\mathrm{S}_{\text {period3 }}=\frac{1}{2}
$$

The conferencing links are not used at all,

$$
\begin{aligned}
& \mu_{\mathrm{Tx}, \text { Period } 3}=0 \\
& \mu_{\mathrm{Rx}, \text { Period } 3}=0 .
\end{aligned}
$$

4) Analysis of Overall Scheme: We first analyse the communication over the conferencing links. Under assumption (22), there is Tx-conferencing in periods 1 and 2. Given the length of the periods in (23), and the Tx-conferencing rates in periods 1 and 2, (38) and (41), in total each Tx-conferencing link is used at rate not exceeding

$$
\begin{aligned}
& \frac{N_{1}}{n} \mu_{\mathrm{Tx}} \text {,period } 1+\frac{N_{2}}{n} \mu_{\mathrm{Tx}, \text { period2 }} \\
& =\left(\frac{N_{1}}{n} \cdot \frac{\kappa_{\mathrm{Tx}}}{2 \kappa_{\mathrm{Tx}}+2 \kappa_{\mathrm{Rx}}+2}\right. \\
& \left.\quad+\frac{N_{2}}{n} \cdot \frac{\kappa_{\mathrm{Tx}}}{2 \kappa_{\mathrm{Tx}}+2}\right) \cdot \frac{1}{2} \log (1+P) \\
& \leq\left(\frac{\mu_{\mathrm{Rx}}}{\kappa_{\mathrm{Rx}}}\left(2 \kappa_{\mathrm{Tx}}+2 \kappa_{\mathrm{Rx}}+2\right) \frac{\kappa_{\mathrm{Tx}}}{2 \kappa_{\mathrm{Tx}}+2 \kappa_{\mathrm{Rx}}+2}\right. \\
& \left.\quad+\left(\frac{\mu_{\mathrm{Tx}}}{\kappa_{\mathrm{Tx}}}-\frac{\mu_{\mathrm{Rx}}}{\kappa_{\mathrm{Rx}}}\right)\left(2 \kappa_{\mathrm{Tx}}+2\right) \frac{\kappa_{\mathrm{Tx}}}{2 \kappa_{\mathrm{Tx}}+2}\right) \cdot \frac{1}{2} \log (1+P) \\
& \leq \mu_{\mathrm{Tx}} \cdot \frac{1}{2} \log (1+P)=R_{\mathrm{Tx}} .
\end{aligned}
$$

Our overall scheme thus respects the Tx-conferencing rate constraints in (6).

The Rx-conferencing links are used only in period 1 at rate not exceeding (39). Thus, considering the length of this period 1 in (23), in total each receiver conferencing link is used at a rate not exceeding

$$
\begin{aligned}
\frac{N_{1}}{n} \mu_{\mathrm{Rx}, \text { period } 1} & =\frac{N_{1}}{n} \cdot \frac{\kappa_{\mathrm{Rx}}}{2 \kappa_{\mathrm{Tx}}+2 \kappa_{\mathrm{Rx}}+2} \cdot \frac{1}{2} \log (1+P) \\
& \leq \mu_{\mathrm{Rx}} \cdot \frac{1}{2} \log (1+P)=R_{\mathrm{Rx}} .
\end{aligned}
$$

Our overall scheme thus also respects the $\mathrm{Rx}$-conferencing rate constraints in (9).

We now analyse the per-user MG achieved by our overall scheme. It is given by

$$
\mathrm{S}=\frac{N_{1}}{n} \mathrm{~S}_{\text {period } 1}+\frac{N_{2}}{n} \mathrm{~S}_{\text {period } 2}+\frac{N_{3}}{n} \mathrm{~S}_{\text {period } 3} .
$$

To evaluate this expression, we distinguish three cases:

1) When

$$
1 \leq \frac{\mu_{\mathrm{Rx}}}{\kappa_{\mathrm{Rx}}}\left(2 \kappa_{\mathrm{Tx}}+2 \kappa_{\mathrm{Rx}}+2\right)
$$

then $N_{1}=n$ and periods 2 and 3 don't exist. In this case,

$$
\mathrm{S}=\mathrm{S}_{\text {period } 1}=\frac{2 \kappa_{\mathrm{Tx}}+2 \kappa_{\mathrm{Rx}}+1}{2 \kappa_{\mathrm{Tx}}+2 \kappa_{\mathrm{Rx}}+2} .
$$

2) When $1>\frac{\mu_{\mathrm{Rx}}}{\kappa_{\mathrm{Rx}}}\left(2 \kappa_{\mathrm{Tx}}+2 \kappa_{\mathrm{Rx}}+2\right)$ and

$$
\begin{aligned}
1 & <\frac{\mu_{\mathrm{Rx}}}{\kappa_{\mathrm{Rx}}}\left(2 \kappa_{\mathrm{Tx}}+2 \kappa_{\mathrm{Rx}}+2\right)+\left(\frac{\mu_{\mathrm{Tx}}}{\kappa_{\mathrm{Tx}}}-\frac{\mu_{\mathrm{Rx}}}{\kappa_{\mathrm{Rx}}}\right)\left(2 \kappa_{\mathrm{Tx}}+2\right) \\
& =2 \mu_{\mathrm{Tx}}+2 \mu_{\mathrm{Rx}}+2 \frac{\mu_{\mathrm{Tx}}}{\kappa_{\mathrm{Tx}}}
\end{aligned}
$$

then

$$
N_{1}=n \frac{\mu_{\mathrm{Rx}}}{\kappa_{\mathrm{Rx}}}\left(2 \kappa_{\mathrm{Tx}}+2 \kappa_{\mathrm{Rx}}+2\right),
$$

$N_{2}=n-N_{1}$ and $N_{3}=0$ (i.e., period 3 does not exist). In this case,

$$
\begin{aligned}
\mathrm{S}= & \frac{N_{1}}{n} \mathrm{~S}_{\text {period } 1}+\left(1-\frac{N_{1}}{n}\right) \mathrm{S}_{\text {period } 2} \\
= & \frac{\mu_{\mathrm{Rx}}}{\kappa_{\mathrm{Rx}}}\left(2 \kappa_{\mathrm{Tx}}+2 \kappa_{\mathrm{Rx}}+2\right) \cdot \frac{2 \kappa_{\mathrm{Tx}}+2 \kappa_{\mathrm{Rx}}+1}{2 \kappa_{\mathrm{Tx}}+2 \kappa_{\mathrm{Rx}}+2} \\
& +\left(1-\frac{\mu_{\mathrm{Rx}}}{\kappa_{\mathrm{Rx}}}\left(2 \kappa_{\mathrm{Tx}}+2 \kappa_{\mathrm{Rx}}+2\right)\right) \frac{2 \kappa_{\mathrm{Tx}}+1}{2 \kappa_{\mathrm{Tx}}+2} \\
= & \frac{2 \kappa_{\mathrm{Tx}}+1+2 \mu_{\mathrm{Rx}}}{2 \kappa_{\mathrm{Tx}}+2} .
\end{aligned}
$$

3) When

$$
1>2 \mu_{\mathrm{Tx}}+2 \mu_{\mathrm{Rx}}+2 \frac{\mu_{\mathrm{Tx}}}{\kappa_{\mathrm{Tx}}},
$$

then

$$
\begin{aligned}
& N_{1}=n \frac{\mu_{\mathrm{Rx}}}{\kappa_{\mathrm{Rx}}}\left(2 \kappa_{\mathrm{Tx}}+2 \kappa_{\mathrm{Rx}}+2\right), \\
& N_{2}=n\left(\frac{\mu_{\mathrm{Tx}}}{\kappa_{\mathrm{Tx}}}-\frac{\mu_{\mathrm{Rx}}}{\kappa_{\mathrm{Rx}}}\right)\left(2 \kappa_{\mathrm{Tx}}+2\right),
\end{aligned}
$$

and

$$
N_{3}=n-N_{1}-N-2>0 .
$$


In this case,

$$
\begin{aligned}
\mathrm{S}= & \frac{N_{1}}{n} \mathrm{~S}_{\text {period } 1}+\frac{N_{2}}{n} \mathrm{~S}_{\text {period } 2} \\
& +\left(1-\frac{N_{1}}{n}-\frac{N_{2}}{n}\right) \mathrm{S}_{\text {period } 3} \\
= & \frac{1+2 \mu_{\mathrm{Tx}}+2 \mu_{\mathrm{Rx}}}{2} .
\end{aligned}
$$

Combining all these findings proves Theorem 2.

\section{Converses to Theorems 1 And 2}

We will prove the following five bounds

- For unlimited number of conferencing rounds:

$$
\mathrm{S}_{\infty}(\boldsymbol{\mu}) \leq 1
$$

- For unlimited number of conferencing rounds:

$$
\mathrm{S}_{\infty}(\boldsymbol{\mu}) \leq \frac{2 \mu_{\mathrm{Tx}}+2 \mu_{\mathrm{Rx}}+1}{2} .
$$

- For at most $\kappa_{\mathrm{Tx}}$ transmitter-conferencing rounds (irrespective of the number of receiver-conferencing rounds $\left.\kappa_{\mathrm{Rx}}\right)$ :

$$
\mathrm{S}\left(\kappa_{\mathrm{Tx}}, \kappa_{\mathrm{Rx}}, \mu_{\mathrm{Tx}}, \mu_{\mathrm{Rx}}\right) \leq \frac{2 \kappa_{\mathrm{Tx}}+2 \mu_{\mathrm{Rx}}+1}{2 \kappa_{\mathrm{Tx}}+2}
$$

- For at most $\kappa_{\mathrm{Rx}}$ receiver-conferencing rounds (irrespective of the number of transmitter-conferencing rounds $\left.\kappa_{\mathrm{Tx}}\right)$ :

$$
\mathrm{S}\left(\kappa_{\mathrm{Tx}}, \kappa_{\mathrm{Rx}}, \mu_{\mathrm{Tx}}, \mu_{\mathrm{Rx}}\right) \leq \frac{2 \kappa_{\mathrm{Rx}}+2 \mu_{\mathrm{Tx}}+1}{2 \kappa_{\mathrm{Rx}}+2} .
$$

- For at ost $\kappa_{\mathrm{Tx}}$ transmitter-conferencing rounds and at $\kappa_{\mathrm{Rx}}$ receiver-conferencing rounds:

$$
\mathrm{S}\left(\kappa_{\mathrm{Tx}}, \kappa_{\mathrm{Rx}}, \mu_{\mathrm{Tx}}, \mu_{\mathrm{Rx}}\right) \leq \frac{2 \kappa_{\mathrm{Tx}}+2 \kappa_{\mathrm{Rx}}+1}{2 \kappa_{\mathrm{Tx}}+2 \kappa_{\mathrm{Rx}}+2} .
$$

Since for any $\boldsymbol{\mu} \geq 0$ :

$$
\mathrm{S}(\kappa, \mu) \leq \mathrm{S}_{\infty}(\mu)
$$

above five bounds conclude the proofs of the two converses to Theorems 1 and 2.

We prove these bounds using a general lemma that we state in the next subsection. The lemma provides a MAC-type upper bound, similarly to Sato's MAC-bound for interference channels without cooperation [40], [41] and similarly to the Dynamic MAC-Lemma for interference networks with message cognition and clustered decoding in [30, Lemma 9].

\section{A. MAC-Lemma for Interference Networks With Conferencing}

Consider a $K$-user interference network with transmitterand receiver-conferencing between adjacent users as described in Section II.

Given a set of indices $\mathcal{J} \subseteq \mathcal{K}:=\{1, \ldots, K\}$, define the following sets of inputs, outputs, and messages:

$$
\begin{aligned}
\mathbf{X}_{\mathcal{J}}^{n} & :=\left\{X_{k}^{n}, k \in \mathcal{J}\right\}, \\
\mathbf{Y}_{\mathcal{J}}^{n} & :=\left\{Y_{k}^{n}, k \in \mathcal{J}\right\}, \\
\mathbf{Z}_{\mathcal{J}}^{n} & :=\left\{Z_{k}^{n}, k \in \mathcal{J}\right\}, \\
\mathbf{M}_{\mathcal{J}}^{n} & :=\left\{M_{k}, k \in \mathcal{J}\right\}, \\
\hat{\mathbf{M}}_{\mathcal{J}}^{n} & :=\left\{\hat{M}_{k}, k \in \mathcal{J}\right\} .
\end{aligned}
$$

Consider arbitrary sets $\mathcal{J}_{1}, \mathcal{J}_{2}$ satisfying

$$
\mathcal{J}_{1} \subseteq \mathcal{J}_{2} \subseteq \mathcal{K}
$$

Let $\mathcal{L}_{\text {left-outputs }}\left(\mathcal{J}_{1}, \mathcal{J}_{2}\right)$ denote the subset of indices in $\mathcal{J}_{2}$ that have an immediate left-neighbour not in $\mathcal{J}_{2}$ and have at least one right-neighbour at distance less than $\kappa_{\mathrm{Rx}}-1$ that lies in $\mathcal{J}_{1}$ :

$$
\begin{aligned}
\mathcal{L}_{\text {left-outputs }}\left(\mathcal{J}_{1}, \mathcal{J}_{2}\right) & \\
:= & \left\{k \in \mathcal{J}_{2}:\left(k-1 \notin \mathcal{J}_{2}\right. \text { and }\right. \\
& \left.\left.\quad\left\{k+1, k+2, \ldots, k+\kappa_{\mathrm{Rx}}-1\right\} \cap \mathcal{J}_{1} \neq \emptyset\right)\right\},
\end{aligned}
$$

Now let $\mathcal{L}_{\text {right-outputs }}\left(\mathcal{J}_{1}, \mathcal{J}_{2}\right)$ denote the subset of indices in $\mathcal{J}_{2}$ that have an immediate right-neighbour not in $\mathcal{J}_{2}$ and have at least one left-neighbour at distance less than $\kappa_{\mathrm{Rx}}-1$ that lies in $\mathcal{J}_{1}$ :

$$
\begin{aligned}
\mathcal{L}_{\text {right-outputs }}\left(\mathcal{J}_{1}, \mathcal{J}_{2}\right) & \\
:= & \left\{k \in \mathcal{J}_{2}: \quad\left(k+1 \notin \mathcal{J}_{2}\right. \text { and }\right. \\
& \left.\left.\quad\left\{k-\kappa_{\mathrm{Rx}}+1, k-\kappa_{\mathrm{Rx}}+2, \ldots, k-1\right\} \cap \mathcal{J}_{1} \neq \emptyset\right)\right\},
\end{aligned}
$$

In (59), the indices that are not in $\{1, \ldots, K\}$ should be ignored. Moreover, when the number of receiver-conferencing rounds is unlimited, then in above definitions $\kappa_{\mathrm{Rx}}$ needs to be replaced by $K$.

For short we will simply write $\mathcal{L}_{\text {left-outputs }}$ and $\mathcal{L}_{\text {right-outputs }}$ when the sets $\mathcal{J}_{1}$ and $\mathcal{J}_{2}$ are clear from the context.

Observation 1: Let $\mathcal{J}_{\text {messages }}$ and $\mathcal{J}_{\text {outputs }}$ be two sets satisfying

$$
\mathcal{J}_{\text {messages }} \subseteq \mathcal{J}_{\text {outputs }} \subseteq \mathcal{K} .
$$

Irrespective of the chosen conferencing, encoding, and decoding functions, it is possible to reproduce the receivers' guesses of source messages $\hat{\mathbf{M}}_{\mathcal{J}_{\text {messages }}}$, based only on the following three sets:

1) The set of output signals $\mathbf{Y}_{\mathcal{J}_{\text {outputs }}}^{n}$;

2) the left-to-right receiver-conferencing signals

$$
\begin{aligned}
& \left\{V_{k-1 \rightarrow k}^{(j)}: \quad j \in\left\{1, \ldots, \kappa_{\mathrm{Rx}}\right\},\right. \\
& \left.\quad k \in \mathcal{L}_{\text {left-outputs }}\left(\mathcal{J}_{\text {messages }}, \mathcal{J}_{\text {outputs }}\right)\right\} ;
\end{aligned}
$$

3) the right-to-left receiver-conferencing signals

$$
\begin{aligned}
& \left\{V_{k+1 \rightarrow k}^{(j)}: \quad j \in\left\{1, \ldots, \kappa_{\mathrm{Rx}}\right\},\right. \\
& \left.\quad k \in \mathcal{L}_{\text {right-outputs }}\left(\mathcal{J}_{\text {messages }}, \mathcal{J}_{\text {outputs }}\right)\right\},
\end{aligned}
$$

In fact, since the number of receiver-conferencing rounds is limited to $\kappa_{\mathrm{Rx}}$, from above outputs and conferencing signals it is possible to reconstruct all conferencing messages sent to receivers in $\mathcal{J}_{\text {messages }}$.

Similarly, for any sets of indices $\mathcal{J}_{1}$ and $\mathcal{J}_{2}$ satisfying (58), let $\mathcal{L}_{\text {left-messages }}$ denote the subset of indices in $\mathcal{J}_{2}$ that have 
an immediate left-neighbour not in $\mathcal{J}_{2}$ and have at least one right-neighbour at distance less than $\kappa_{\mathrm{Tx}}-1$ that lies in $\mathcal{J}_{1}$ :

$$
\begin{aligned}
\mathcal{L}_{\text {left-messages }}\left(\mathcal{J}_{1}, \mathcal{J}_{2}\right) & := \\
& \left\{k \in \mathcal{J}_{2}:\left(k-1 \notin \mathcal{J}_{2}\right. \text { and }\right. \\
& \left.\left.\left\{k+1, k+2, \ldots, k+\kappa_{\mathrm{Tx}}-1\right\} \cap \mathcal{J}_{1} \neq \emptyset\right)\right\},
\end{aligned}
$$

Also, let $\mathcal{L}_{\text {right-messages }}\left(\mathcal{J}_{1}, \mathcal{J}_{2}\right)$ denote the subset of indices in $\mathcal{J}_{2}$ that have an immediate right-neighbour not in $\mathcal{J}_{2}$ and have at least one left-neighbour at distance less than $\kappa_{\mathrm{Tx}}-1$ that lies in $\mathcal{J}_{1}$ :

$$
\begin{aligned}
\mathcal{L}_{\text {right-messages }}\left(\mathcal{J}_{1}, \mathcal{J}_{2}\right) & \\
:= & \left\{k \in \mathcal{J}_{2}:\left(k+1 \notin \mathcal{J}_{2}\right. \text { and }\right. \\
\quad & \left.\left.\left\{k-\kappa_{\mathrm{Tx}}+1, k-\kappa_{\mathrm{Tx}}+2, \ldots, k-1\right\} \cap \mathcal{J}_{1} \neq \emptyset\right)\right\} .
\end{aligned}
$$

In these definitions, indices that are not in $\{1, \ldots, K\}$ should again be ignored. Also, when the number of transmitterconferencing rounds is unlimited, then $\kappa_{\mathrm{Tx}}$ needs to be replaced by $K$.

Observation 2: Let $\mathcal{J}_{\text {messages }}$ and $\mathcal{J}_{\text {inputs }}$ be two sets satisfying

$$
\mathcal{J}_{\text {inputs }} \subseteq \mathcal{J}_{\text {messages }} \subseteq \mathcal{K} .
$$

Irrespective of the chosen conferencing, encoding, and decoding functions, it is possible to reproduce the channel inputs $\mathbf{X}_{\mathcal{J}_{\text {inputs }}^{n}}^{n}$, based only on the following three sets:

1) The set of messages $\mathbf{M}_{\mathcal{J}_{\text {messages }}}$;

2) the left-to-right transmitter-conferencing signals

$$
\begin{aligned}
& \left\{U_{k-1 \rightarrow k}^{(j)}: j \in\left\{1, \ldots, \kappa_{\mathrm{Tx}}\right\},\right. \\
& \left.\quad k \in \mathcal{L}_{\text {left-messages }}\left(\mathcal{J}_{\text {inputs }}, \mathcal{J}_{\text {messages }}\right)\right\} ;
\end{aligned}
$$

3) the right-to-left transmitter-conferencing signals

$$
\begin{aligned}
& \left\{U_{k+1 \rightarrow k}^{(j)}: j \in\left\{1, \ldots, \kappa_{\mathrm{Tx}}\right\},\right. \\
& \left.\quad k \in \mathcal{L}_{\text {right-messages }}\left(\mathcal{J}_{\text {inputs }}, \mathcal{J}_{\text {messages }}\right)\right\} .
\end{aligned}
$$

In fact, since the number of transmitter-conferencing rounds is limited to $\kappa_{\mathrm{Tx}}$, from above messages and conferencing signals it is possible to reconstruct all conferencing messages sent to transmitters in $\mathcal{J}_{\text {inputs. }}$.

Above two observations allow us to obtain the following lemma.

Lemma 1 (MAC-Bound for Interference Networks With Conferencing): Consider an interference network with $K$ transmitter-receiver pairs and where adjacent transmitters and receivers can hold conferencing communications over noisefree pipes of prelogs $\mu_{\mathrm{Tx}}$ and $\mu_{\mathrm{Rx}}$ as described in Section II.

Let $\mathcal{J}_{\text {inputs }}, \mathcal{J}_{\text {messages }}$, and $\mathcal{J}_{\text {outputs }}$ be three subsets of $\mathcal{K}$ satisfying

$$
\mathcal{J}_{\text {inputs }} \subseteq \mathcal{J}_{\text {messages }} \subseteq \mathcal{J}_{\text {outputs }} \subseteq \mathcal{K},
$$

and let $G_{0}^{n}, G_{1}^{n}, \ldots, G_{\ell}^{n}$ be $\ell+1$ genie-signals, for some $\ell \in \mathbb{Z}$, independent of the messages $M_{1}, \ldots, M_{K}$ and so that

$$
\frac{1}{n} h\left(\mathbf{Z}_{\mathcal{J}_{\text {outputs }}} \mid G_{0}^{n}, G_{1}^{n}, \ldots, G_{\ell}^{n}\right) \geq-o(\log (P)),
$$

where $o(\cdot)$ denotes the "little-o" Landau symbol.

If for any sequence of encoding, decoding, and conferencing functions, there exist for each blocklength $n$ functions $\left\{\xi_{k}^{(n)}, k \in \mathcal{K}\right\}$ so that

$Y_{k}^{n}=\xi_{k}^{(n)}\left(\mathbf{X}_{\mathcal{J}_{\text {inputs }}}^{n}, \mathbf{Y}_{\mathcal{J}_{\text {outputs }}}^{n}, G_{0}^{n}, G_{1}^{n}, \ldots, G_{\ell}^{n}\right), \quad k \in \mathcal{K}$,

then the MG is upper-bounded as

$$
\begin{aligned}
\varlimsup_{P \rightarrow \infty} \frac{\mathrm{C}_{\Sigma}(\kappa, \mu, P)}{\frac{1}{2} \log P} \leq & +\mu_{\mathrm{Tx}}\left(\left|\mathcal{L}_{\text {left-messages }}\right|+\left|\mathcal{L}_{\text {right-messages }}\right|\right) \\
& +\mu_{\mathrm{Rx}}\left(\left|\mathcal{L}_{\text {left-outputs }}\right|+\left|\mathcal{L}_{\text {right-outputs }}\right|\right) \\
& +\left|\mathcal{J}_{\text {outputs }}\right|
\end{aligned}
$$

Proof: Define a super-receiver that observes

- The set of output signals $\mathbf{Y}_{\mathcal{J}_{\text {outputs }}}^{n}$;

- The genie-informations $G_{0}^{n}, G_{1}^{n}, \ldots, G_{\ell}^{n}$;

- The left-to-right receiver-conferencing signals

$$
\begin{aligned}
\mathbf{V}_{\mathcal{L}_{\text {left-messages }}:=} & \left\{V_{k-1 \rightarrow k}^{(j)}: \quad j \in\left\{1, \ldots, \kappa_{\mathrm{Rx}}\right\},\right. \\
& \left.k \in \mathcal{L}_{\text {left-outputs }}\left(\mathcal{J}_{\text {messages }}, \mathcal{J}_{\text {outputs }}\right)\right\} ;
\end{aligned}
$$

- The right-to-left receiver-conferencing signals

$$
\begin{aligned}
\mathbf{V}_{\mathcal{L}_{\text {right-messages }}:=} & \left\{V_{k+1 \rightarrow k}^{(j)}: j \in\left\{1, \ldots, \kappa_{\mathrm{Rx}}\right\},\right. \\
& \left.k \in \mathcal{L}_{\text {right-outputs }}\left(\mathcal{J}_{\text {messages }}, \mathcal{J}_{\text {outputs }}\right)\right\} ;
\end{aligned}
$$

- The left-to-right transmitter-conferencing signals

$$
\begin{aligned}
\mathbf{U}_{\mathcal{L}_{\text {left-inputs }}:=} & \left\{U_{k-1 \rightarrow k}^{(j)}: j \in\left\{1, \ldots, \kappa_{\mathrm{Tx}}\right\},\right. \\
& \left.k \in \mathcal{L}_{\text {left-messages }}\left(\mathcal{J}_{\text {inputs }}, \mathcal{J}_{\text {messages }}\right)\right\} ;
\end{aligned}
$$

- The right-to-left transmitter-conferencing signals

$$
\begin{aligned}
\mathbf{U}_{\mathcal{L}_{\text {right-inputs }}:=} & \left\{U_{k+1 \rightarrow k}^{(j)}: \quad j \in\left\{1, \ldots, \kappa_{\mathrm{Tx}}\right\},\right. \\
& \left.k \in \mathcal{L}_{\text {right-messages }}\left(\mathcal{J}_{\text {inputs }}, \mathcal{J}_{\text {messages }}\right)\right\} .
\end{aligned}
$$

We first prove that this super-receiver can correctly decode all messages $M_{1}, \ldots, M_{K}$ whenever the $K$ original receivers decode them correctly. Fix a blocklength $n$ and conferencing, encoding, and decoding functions at the $K$ original transmitters and receivers. Assume that these original receivers correctly decode their desired messages. The super-receiver proceeds as follows:

1) It reconstructs all conferencing messages to receivers in $\mathcal{J}_{\text {messages }}$ and produces the same guesses of messages $\hat{\mathbf{M}}_{\mathcal{J}_{\text {messages }}}$ as the original receivers. By Observation 1 , this is possible by only using the super-receiver's 


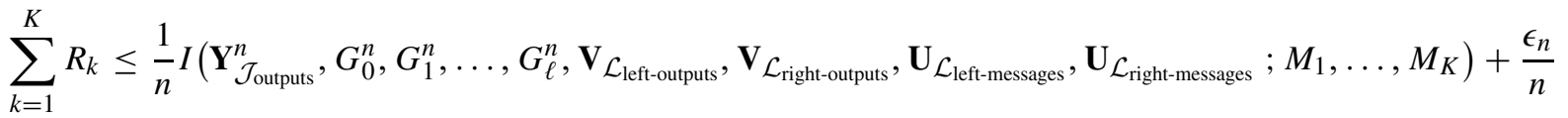

$$
\begin{aligned}
& \leq \frac{1}{n} I\left(\mathbf{Y}_{\mathcal{J}_{\text {outputs }}}^{n} ; M_{1}, \ldots, M_{K} \mid G_{0}^{n}, G_{1}^{n}, \ldots, G_{\ell}^{n}\right)
\end{aligned}
$$

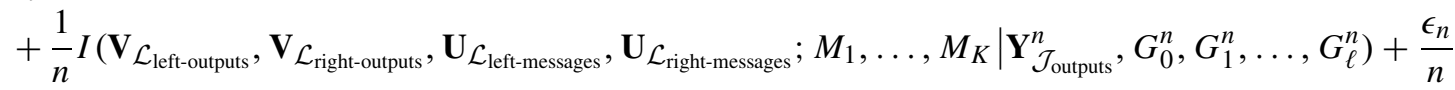

$$
\begin{aligned}
& \leq \frac{1}{n} h\left(\mathbf{Y}_{\mathcal{J}_{\text {outputs }}}^{n}\right)-\frac{1}{n} h\left(\mathbf{Y}_{\mathcal{J}_{\text {outputs }}}^{n} \mid G_{0}^{n}, G_{1}^{n}, \ldots, G_{\ell}^{n}, M_{1}, \ldots, M_{K}\right) \\
& +\frac{1}{n} H\left(\mathbf{V}_{\mathcal{L}_{\text {left-outputs }}}\right)+\frac{1}{n} H\left(\mathbf{V}_{\mathcal{L}_{\text {right-outputs }}}\right)+\frac{1}{n} H\left(\mathbf{U}_{\mathcal{L}_{\text {left-messages }}}\right)+\frac{1}{n} H\left(\mathbf{U}_{\mathcal{L}_{\text {right-messages }}}\right)+\frac{\epsilon_{n}}{n} \\
& \leq \frac{1}{n} h\left(\mathbf{Y}_{\mathcal{J}_{\text {outputs }}}^{n}\right)-\frac{1}{n} h\left(\mathbf{Z}_{\mathcal{J}_{\text {outputs }}}^{n} \mid G_{0}^{n}, G_{1}^{n}, \ldots, G_{\ell}^{n}\right) \\
& +\frac{1}{n} H\left(\mathbf{V}_{\mathcal{L}_{\text {left-outputs }}}\right)+\frac{1}{n} H\left(\mathbf{V}_{\mathcal{L}_{\text {right-outputs }}}\right)+\frac{1}{n} H\left(\mathbf{U}_{\mathcal{L}_{\text {left-messages }}}\right)+\frac{1}{n} H\left(\mathbf{U}_{\mathcal{L}_{\text {right-messages }}}\right)+\frac{\epsilon_{n}}{n} \\
& \leq\left|\mathcal{J}_{\text {outputs }}\right| \cdot \frac{1}{2} \log \left(1+\left(1+\alpha_{\max }\right)^{2} P\right)+o(\log (P)) \\
& +\mu_{\mathrm{Tx}} \frac{1}{2} \log (1+P)\left(\left|\mathcal{L}_{\text {left-outputs }}\right|+\left|\mathcal{L}_{\text {right-outputs }}\right|\right)+\mu_{\mathrm{Rx}} \frac{1}{2} \log (1+P)\left(\left|\mathcal{L}_{\text {left-messages }}\right|+\left|\mathcal{L}_{\text {right-messages }}\right|\right)+\frac{\epsilon_{n}}{n} .
\end{aligned}
$$

outputs $\mathbf{Y}_{\mathcal{J}_{\text {outputs }}}^{n}$ and its receiver-conferencing signals $\mathbf{V}_{\mathcal{L}_{\text {left-messages }}}$ and $\mathbf{V}_{\mathcal{L}_{\text {right-messages }}}$.

2) It then computes all channel inputs $\mathbf{X}_{\mathcal{J}_{\text {inputs }}}^{n}$ produced by the original transmitters. By Observation 2, this is possible whenever the super-receiver's previous decoding step was successful (i.e., whenever the original receivers correctly decode $\hat{\mathbf{M}}_{\mathcal{J}_{\text {messages }}}$ ), because inputs $\mathbf{X}_{\mathcal{J}_{\text {inputs }}}^{n}$ can be computed based only on the super-receiver's transmitterconferencing signals $\mathbf{U}_{\mathcal{L}_{\text {left-inputs }}}$ and $\mathbf{U}_{\mathcal{L}_{\text {right-inputs }}}$ and on messages $\mathbf{M}_{\mathcal{J}_{\text {messages }}}$.

3) It calculates the missing channel outputs $\mathbf{Y}_{\mathcal{K} \backslash \mathcal{J}_{\text {outputs }}}^{n}$ using channel outputs $\mathbf{Y}_{\mathcal{J}_{\text {outputs }}}^{n}$, inputs $\mathbf{X}_{\mathcal{J}_{\text {inputs }}}^{\mathbf{n}}$, and genie-information $G_{0}^{n}, \ldots, G_{\ell}^{n}$, which is possible by the assumption in the lemma.

4) From the entire set of channel outputs $\mathbf{Y}_{\mathcal{K}}^{n}$, it then reconstructs all conferencing messages to receivers in $\mathcal{K} \backslash \mathcal{J}_{\text {messages }}$

5) It finally decodes messages $\hat{\mathbf{M}}_{\mathcal{K}} \backslash \mathcal{J}_{\text {messages }}$ in the same way as the original receivers.

Using this five-steps decoding, the super-receiver decodes correctly whenever the $K$ original receivers decode correctly.

We now bound the rates that are achievable to the constructed super-receiver. Recall that it observes the output signals $\mathbf{Y}_{\mathcal{J}_{\text {outputs }}}^{n}$, the genie-information $G_{0}^{n}, G_{1}^{n}, \ldots, G_{\ell}^{n}$, and the conferencing signals in (68). Let

$$
\alpha_{\max }:=\max _{k=2, \ldots, K}\left|\alpha_{k}\right|
$$

By Fano's inequality, any rate-tuple $\left(R_{1}, \ldots, R_{K}\right)$ that is achievable must satisfy for some sequence $\epsilon_{n} \rightarrow 0$ as $n \rightarrow$ $\infty$ the inequalities leading to (70), as shown at the top of this page. Letting $n \rightarrow \infty$, dividing by $\frac{1}{2} \log (1+P)$, and letting finally $P \rightarrow \infty$ we obtain the desired upper bound in (68).

\section{B. Proof of (53)}

Follows by specializing Lemma 1 to the choice

$$
\mathcal{J}_{\text {inputs }}=\mathcal{J}_{\text {messages }}=\mathcal{J}_{\text {outputs }}=\mathcal{K} \text {. }
$$

There is no genie-information. For this choice, the sets

$\mathcal{L}_{\text {left-messages }}=\mathcal{L}_{\text {right-messages }}=\mathcal{L}_{\text {left-outputs }}=\mathcal{L}_{\text {right-outputs }}=\varnothing$ are all empty, and knowing $\mathbf{Y}_{\mathcal{J}_{\text {outputs }}}^{n}$ one can trivially reconstruct all outputs $Y_{1}^{n}, \ldots, Y_{K}^{n}$. From Lemma 1 we thus obtain the upper bound

$$
\mathrm{S}_{\infty} \leq 1
$$

\section{Proof of (54)}

Follows by specializing Lemma 1 to the genie-information $G_{i}^{n}:=Z_{2(i+1)}^{n}-\alpha_{2(i+1)} Z_{2 i+1}^{n}, \quad i \in\{0,1, \ldots,\lfloor K / 2\rfloor-1\}$, and to the sets

$\mathcal{J}_{\text {inputs }}=\mathcal{J}_{\text {messages }}=\mathcal{J}_{\text {outputs }}=\{2,4,6, \ldots, 2 \cdot\lfloor K / 2\rfloor, K\}$.

So, above sets contain all even indices as well as the last index $K$ in case $K$ is odd. Since the set $\mathbf{Y}_{\mathcal{J}_{\text {outputs }}}^{n}$ contains only every second output sequence, in order to decode messages $\mathbf{M}_{\mathcal{J}_{\text {messages }}}$ one also needs to know all left-toright conferencing messages to receivers in $\{2,4,6, \ldots, K\}$ and all right-to-left conferencing messages to receivers in $\{2,4,6,8, \ldots, 2 \cdot\lfloor K / 2\rfloor-2\}$. Similarly, to reconstruct channel inputs $\mathbf{X}_{\mathcal{J}_{\text {inputs }}}^{n}$ from messages $\mathbf{M}_{\mathcal{J}_{\text {messages }}}$ one also needs to know all left-to-right conferencing messages to transmitters in $\{2,4,6,8, \ldots, K\}$ and all right-to-left conferencing messages to transmitters in $\{2,4,6,8, \ldots, 2 \cdot\lfloor K / 2\rfloor-2\}$. Irrespective of the number of allowed conferencing rounds:

$$
\begin{aligned}
\mathcal{L}_{\text {left-messages }} & =\mathcal{L}_{\text {left-outputs }}=\{2,4,6, \ldots, 2 \cdot\lfloor K / 2\rfloor\}, \\
\mathcal{L}_{\text {right-messages }} & =\mathcal{L}_{\text {right-outputs }}=\{2,4,6, \ldots, 2 \cdot\lfloor K / 2\rfloor-2\} .
\end{aligned}
$$


For each $i \in\{0,1, \ldots,\lfloor K / 2\rfloor-1\}$ it is possible to compute $Y_{2 i+1}^{n}$ from the outputs $Y_{2(i+1)}^{n}$, the inputs $X_{2 i}^{n}$ and $X_{2(i+1)}^{n}$, and the genie-information $G_{i}^{n}$ :

$$
Y_{2 i+1}^{n}=\underbrace{\alpha_{2(i+1)}^{-1}\left(Y_{2(i+1)}^{n}-X_{2(i+1)}^{n}\right)}_{=X_{2 i+1}^{n}+\alpha_{2(i+1)}^{-1} Z_{2(i+1)}^{n}}+\alpha_{2 i+1} X_{2 i}^{n}-\alpha_{2(i+1)}^{-1} G_{i}^{n} .
$$

By Lemma 1 and because

$$
\begin{aligned}
\left|\mathcal{J}_{\text {outputs }}\right| & =K-\lfloor K / 2\rfloor \\
\left|\mathcal{L}_{\text {left-messages }}\right| & =\left|\mathcal{L}_{\text {left-outputs }}\right|=\lfloor K / 2\rfloor \\
\left|\mathcal{L}_{\text {right-messages }}\right| & =\left|\mathcal{L}_{\text {right-outputs }}\right|=\lfloor K / 2\rfloor-1
\end{aligned}
$$

we obtain the desired upper bound

$$
\mathrm{S}_{\infty} \leq \frac{1+2 \mu_{\mathrm{Tx}}+2 \mu_{\mathrm{Rx}}}{2} .
$$

\section{Proof of (55)}

Define

$$
\begin{aligned}
& \beta_{1}:=2\left(\kappa_{\mathrm{Tx}}+1\right) \\
& \gamma_{1}:=\left\lfloor\frac{K-\kappa_{\mathrm{Tx}}-2}{2 \kappa_{\mathrm{Tx}}+2}\right\rfloor .
\end{aligned}
$$

We specialize Lemma 1 to the choice of index sets

$$
\begin{aligned}
\mathcal{J}_{\text {inputs }} & =\left\{g \beta_{1}+\kappa_{\mathrm{Tx}}+2\right\}_{g=0}^{\gamma_{1}}, \\
\mathcal{J}_{\text {messages }} & =\mathcal{J}_{\text {outputs }}=\mathcal{K} \backslash\left\{g \beta_{1}+1\right\}_{g=0}^{\gamma_{1}} .
\end{aligned}
$$

One can decode messages $\mathbf{M}_{\mathcal{J}_{\text {messages }}}$ if one knows outputs $\mathbf{Y}_{\mathcal{J}_{\text {outputs }}}^{n}$ as well as all left-to-right conferencing messages to receivers in $\left\{g \beta_{1}+2\right\}_{g=0}^{\gamma_{1}}$ and all right-to-left conferencing messages to receivers in $\left\{g \beta_{1}\right\}_{g=1}^{\gamma_{1}}$. In fact, with these outputs and conferencing messages it is possible to compute all conferencing messages to receivers in $\mathcal{J}_{\text {messages }}$. Therefore,

$$
\begin{aligned}
\mathcal{L}_{\text {left-outputs }} & =\left\{g \beta_{1}+2\right\}_{g=0}^{\gamma_{1}}, \\
\mathcal{L}_{\text {right-outputs }} & =\left\{g \beta_{1}\right\}_{g=1}^{\gamma_{1}} .
\end{aligned}
$$

From Messages $\mathbf{M}_{\mathcal{J}_{\text {messages }}}$ one can reconstruct all conferencing messages to transmitters in $\mathcal{J}_{\text {inputs }}$, and thus also their corresponding inputs $\mathbf{X}_{\mathcal{J}_{\text {inputs }}}^{n}$. Therefore,

$$
\mathcal{L}_{\text {left-messages }}=\mathcal{L}_{\text {right-messages }}=\emptyset .
$$

We choose $\ell=\gamma_{1}$ and the genie-informations:

$$
G_{0}^{n}=Z_{1}^{n}+\sum_{\nu=1}^{\kappa_{\mathrm{Tx}}}\left(\prod_{j=1}^{\nu} \frac{-1}{\alpha_{1+j}}\right) Z_{1+\nu}^{n}
$$

and for $g \in\left\{1, \ldots, \gamma_{1}\right\}$ :

$$
\begin{aligned}
G_{g}^{n}= & Z_{1+g \beta_{1}}^{n}+\sum_{\nu=1}^{\kappa_{\mathrm{Tx}}}\left(\prod_{j=1}^{\nu} \frac{-1}{\alpha_{g} \beta_{1}+1+j}\right) Z_{1+g \beta_{1}+\nu}^{n} \\
& +\sum_{\nu=1}^{\kappa_{\mathrm{TX}}}\left(\prod_{j=1}^{\nu}\left(-\alpha_{g \beta_{1}+1+j-\nu}\right)\right) Z_{1+g \beta_{1}-\nu}^{n}
\end{aligned}
$$

Notice that from the genie-informations $G_{0}^{n}, G_{1}^{n}, \ldots, G_{\ell}^{n}$, the inputs $\mathbf{X}_{\mathcal{J}_{\text {inputs }}}^{n}$, and the outputs $\mathbf{Y}_{\mathcal{J}_{\text {oupputs }}}^{n}$ one can reconstruct the remaining outputs $\mathbf{Y}_{\mathcal{K} \backslash \mathcal{J}_{\text {outputs }}^{n}}=\left\{Y_{g \beta_{1}+1}^{n}\right\}_{g=0}^{\gamma_{1}}$. In fact,

$$
\begin{aligned}
Y_{1}^{n}= & -\sum_{\nu=1}^{\kappa_{\mathrm{Tx}}+1}\left(\prod_{j=1}^{\nu} \frac{-1}{\alpha_{1+j}}\right) Y_{1+\nu}^{n} \\
& +\left(\prod_{j=1}^{\kappa_{\mathrm{Tx}}+1} \frac{-1}{\alpha_{1+j}}\right) X_{\kappa_{\mathrm{Tx}}+2}^{n}+G_{0}^{n}
\end{aligned}
$$

and, for $g \in\left\{1, \ldots, \gamma_{1}\right\}$,

$$
\begin{aligned}
Y_{1+g \beta_{1}}^{n} & G_{g}^{n}-\sum_{\nu=1}^{\kappa_{\mathrm{Tx}}+1}\left(\prod_{j=1}^{\nu} \frac{-1}{\alpha_{g} \beta_{1}+1+j}\right) Y_{1+g \beta_{1}+v}^{n} \\
& -\sum_{\nu=1}^{\kappa_{\mathrm{Tx}}}\left(\prod_{j=1}^{v}\left(-\alpha_{g \beta_{1}+1+j-v}\right)\right) Y_{1+g \beta_{1}-\nu}^{n} \\
& +\left(\prod_{j=1}^{t_{\ell}+r_{\ell}+1} \frac{-1}{\alpha_{g} \beta_{1}+1+j}\right) X_{\kappa_{\mathrm{TX}}+2+g \beta_{1}}^{n} \\
& -\left(\prod_{j=0}^{\kappa_{\mathrm{Tx}}}\left(-\alpha_{g \beta_{1}+1+j-\kappa_{\mathrm{Tx}}}\right)\right) X_{\kappa_{\mathrm{TX}}+2+(g-1) \beta_{1}}^{n} .
\end{aligned}
$$

Since

$$
\begin{aligned}
\left|\mathcal{J}_{\text {outputs }}\right| & =K-\gamma_{1}-1=K-\left\lfloor\frac{K-\kappa_{\mathrm{Tx}}-2}{2 \kappa_{\mathrm{Tx}}+2}\right\rfloor-1, \\
\left|\mathcal{L}_{\text {left-messages }}\right| & =\left|\mathcal{L}_{\text {right-messages }}\right|=0, \\
\left|\mathcal{L}_{\text {left-outputs }}\right| & =\gamma_{1}+1=\left\lfloor\frac{K-\kappa_{\mathrm{Tx}}-2}{2 \kappa_{\mathrm{Tx}}+2}\right\rfloor+1 \\
\left|\mathcal{L}_{\text {right-outputs }}\right| & =\gamma_{1}=\left\lfloor\frac{K-\kappa_{\mathrm{Tx}}-2}{2 \kappa_{\mathrm{Tx}}+2}\right\rfloor,
\end{aligned}
$$

we obtain the desired upper bound in (55) by applying Lemma 1 to above choices.

\section{E. Proof of (56)}

Define

$$
\begin{aligned}
& \beta_{2}:=2\left(\kappa_{\mathrm{Rx}}+1\right) \\
& \gamma_{2}:=\left\lfloor\frac{K-\kappa_{\mathrm{Rx}}-2}{2 \kappa_{\mathrm{Rx}}+2}\right\rfloor .
\end{aligned}
$$

We specialize Lemma 1 to the choice of index sets

$$
\begin{aligned}
\mathcal{J}_{\text {messages }} & =\mathcal{J}_{\text {inputs }}=\left\{g \beta_{2}+\kappa_{\mathrm{Rx}}+2\right\}_{g=0}^{\gamma_{2}} \\
\mathcal{J}_{\text {outputs }} & =\mathcal{K} \backslash\left\{g \beta_{2}+1\right\}_{g=0}^{\gamma_{2}},
\end{aligned}
$$

and the genie-informations $G_{0}^{n}, G_{1}^{n}, \ldots, G_{\gamma_{2}}^{n}$ :

$$
G_{0}^{n}=Z_{1}^{n}+\sum_{\nu=1}^{\kappa_{\mathrm{Rx}}}\left(\prod_{j=1}^{\nu} \frac{-1}{\alpha_{1+j}}\right) Z_{1+v}^{n},
$$


and for $g \in\left\{1, \ldots, \gamma_{2}\right\}$ :

$$
\begin{aligned}
G_{g}^{n}= & Z_{1+g \beta_{2}}^{n}+\sum_{\nu=1}^{\kappa_{\mathrm{Rx}}}\left(\prod_{j=1}^{\nu} \frac{-1}{\alpha_{g \beta_{2}+1+j}}\right) Z_{1+g \beta_{2}+\nu}^{n} \\
& +\sum_{\nu=1}^{\kappa_{\mathrm{Rx}}}\left(\prod_{j=1}^{\nu}\left(-\alpha_{g \beta_{2}+1+j-\nu}\right)\right) Z_{1+g \beta_{2}-\nu}^{n}
\end{aligned}
$$

From outputs $\mathbf{Y}_{\mathcal{J}_{\text {outputs }}}^{n}$, one can decode messages $\mathbf{M}_{\mathcal{J}_{\text {messages }}}$. Therefore,

$$
\mathcal{L}_{\text {left-outputs }}=\mathcal{L}_{\text {right-outputs }}=\emptyset .
$$

If one knows all messages $\mathbf{M}_{\mathcal{J}_{\text {messages }}}$, all left-conferencing messages to transmitters in $\left\{g \beta_{2}+2\right\}_{g=0}^{\gamma / 2}$, and all right-to-left conferencing messages to transmitters in $\left\{g \beta_{2}+2\right\}_{g=1}^{\gamma_{2}}$, then one can compute all channel inputs $\mathbf{X}_{\mathcal{J}_{\text {inputs }}}^{n}$. Therefore,

$$
\begin{aligned}
\mathcal{L}_{\text {left-messages }} & =\left\{g \beta_{2}+2\right\}_{g=0}^{\gamma_{2}}, \\
\mathcal{L}_{\text {right-messages }} & =\left\{g \beta_{2}\right\}_{g=1}^{\gamma_{2}} .
\end{aligned}
$$

Notice that from the genie-informations $G_{0}^{n}, G_{1}^{n}, \ldots, G_{\ell}^{n}$, the inputs $\mathbf{X}_{\mathcal{J}_{\text {inputs }}}^{n}$, and the outputs $\mathbf{Y}_{\mathcal{J}_{\text {outputs }}}^{n}$ one can reconstruct the remaining outputs $\mathbf{Y}_{\mathcal{K} \backslash \mathcal{J}_{\text {outputs }}}=\left\{Y_{g \beta_{2}+1}^{n}\right\}_{g=0}^{\gamma_{2}}$. (This can be proved following similar steps as in (72).)

Since

$$
\begin{aligned}
\left|\mathcal{J}_{\text {outputs }}\right| & =K-\gamma_{2}-1=K-\left\lfloor\frac{K-\kappa_{\mathrm{Rx}}-2}{2 \kappa_{\mathrm{Rx}}+2}\right\rfloor-1, \\
\left|\mathcal{L}_{\text {left-messages }}\right| & =\gamma_{2}+1=\left\lfloor\frac{K-\kappa_{\mathrm{Rx}}-2}{2 \kappa_{\mathrm{Rx}}+2}\right\rfloor+1, \\
\left|\mathcal{L}_{\text {right-messages }}\right| & =\gamma_{2}=\left\lfloor\frac{K-\kappa_{\mathrm{Rx}}-2}{2 \kappa_{\mathrm{Rx}}+2}\right\rfloor, \\
\left|\mathcal{L}_{\text {left-outputs }}\right| & =\left|\mathcal{L}_{\text {right-outputs }}\right|=0,
\end{aligned}
$$

we can obtain the desired upper bound (56) by applying Lemma 1 to above choices.

\section{F. Proof of (57)}

Define

$$
\begin{aligned}
& \beta_{3}:=2\left(\kappa_{\mathrm{Tx}}+\kappa_{\mathrm{Rx}}+1\right) \\
& \gamma_{3}:=\left\lfloor\frac{K-\kappa_{\mathrm{Tx}}-\kappa_{\mathrm{Rx}}-2}{2 \kappa_{\mathrm{Tx}}+2 \kappa_{\mathrm{Rx}}+2}\right\rfloor .
\end{aligned}
$$

The desired upper bound (57) can be proved by applying Lemma 1 to the choice of index sets

$$
\begin{aligned}
\mathcal{J}_{\text {messages }} & =\mathcal{J}_{\text {inputs }}=\left\{g \beta_{3}+\kappa_{\mathrm{Tx}}+\kappa_{\mathrm{Rx}}+2\right\}_{g=0}^{\gamma_{3}} \\
\mathcal{J}_{\text {outputs }} & =\mathcal{K} \backslash\left\{g \beta_{3}+1\right\}_{g=0}^{\gamma_{3}},
\end{aligned}
$$

and genie-informations $G_{0}^{n}, G_{1}^{n}, \ldots, G_{\gamma_{3}}^{n}$, where

$$
G_{0}^{n}=Z_{1}^{n}+\sum_{\nu=1}^{\kappa_{\mathrm{Tx}}+\kappa_{\mathrm{Rx}}}\left(\prod_{j=1}^{\nu} \frac{-1}{\alpha_{1+j}}\right) Z_{1+\nu}^{n},
$$

and for $g \in\left\{1, \ldots, \gamma_{3}\right\}$ :

$$
\begin{aligned}
G_{g}^{n}= & Z_{1+g \beta_{3}}^{n}+\sum_{\nu=1}^{\kappa_{\mathrm{Tx}}+\kappa_{\mathrm{Rx}}}\left(\prod_{j=1}^{\nu} \frac{-1}{\alpha_{g \beta_{3}+1+j}}\right) Z_{1+g \beta_{3}+v}^{n} \\
& +\sum_{\nu=1}^{\kappa_{\mathrm{Tx}}+\kappa_{\mathrm{Rx}}}\left(\prod_{j=1}^{v}\left(-\alpha_{g \beta_{3}+1+j-v}\right)\right) Z_{1+g \beta_{3}-v}^{n} .
\end{aligned}
$$

Alternatively, upper bound (57) follows from the following argument. Let us suppose that a genie provides each transmitter the source messages of the $\kappa_{\mathrm{Tx}}$ transmitters on its left and right; that is, transmitter $k$ is given source messages

$$
\left(M_{k-\kappa_{\mathrm{Tx}}}, \ldots, M_{k-1}\right) \text { and }\left(M_{k+1}, \ldots, M_{k+\kappa_{\mathrm{Tx}}}\right),
$$

where messages with indices outside $\{1, \ldots, K\}$ should be ignored.

Let us also suppose that the genie provides to each receiver the exact channel outputs at the $\kappa_{\mathrm{Rx}}$ receivers on its left and right; that is, receiver $k$ is given

$$
\left(Y_{k-\kappa_{\mathrm{Rx}}}^{n}, \ldots, Y_{k-1}^{n}\right) \text { and }\left(Y_{k+1}^{n}, \ldots, Y_{k+\kappa_{\mathrm{Rx}}}^{n}\right),
$$

where outputs with indices outside $\{1, \ldots, K\}$ should also be ignored. Lapidoth et al. [30, Corollary 2] established that the per-user MG of this genie-aided problem is given by $\left(2 \kappa_{\mathrm{Tx}}+\right.$ $\left.2 \kappa_{\mathrm{Rx}}+1\right) /\left(2 \kappa_{\mathrm{Tx}}+2 \kappa_{\mathrm{Rx}}+2\right)$. Since the per-user MG of the genie-aided problem cannot be smaller than $S(\kappa, \mu)$ in the problem at hand, we obtain upper bound (57).

\section{SUMmARY AND CONCLUSION}

We quantify how the per-user MG of Wyner's soft-handoff interference network increases with the number of permitted Tx- and Rx-conferencing rounds. We identify three regimes. When the conferencing links are of low rate, then the peruser MG does not depend on the number of permitted conferencing rounds; a single round of non-interactive conferencing suffices. When the conferencing links are of moderate rate, then an increasing finite number $>1$ of conferencing rounds is needed. Finally, when the conferencing links are of high rate, then every additional conferencing round increases the per-user MG.

Determining the smallest number of conferencing rounds that attains the asymptotic MG per user is of practical interest, because it allows to limit implementation complexity.

Intuitively, increasing the number of $\mathrm{Tx}^{-}$and $\mathrm{Rx}$-conferencing rounds in an interference network can be beneficial, because information about a given transmit message or about a given receive signal can be spread over a larger part of the network. This is important for iterative interference mitigation techniques (like successive dirtypaper coding at the transmitters and successive interference cancellation at the receivers), where interference-mitigation information precisely needs to propagate over the network.

To avoid propagating interference beyond what can be mitigated with the number of permitted conferencing rounds, in our scheme we periodically deactivate transmitters. This splits the large network into smaller subnets. Over each of these subnets we employ a coding scheme that smartly 
combines transmitter and receiver interference-mitigation techniques so as to fully cancel all the interference in the subnet.

The conferencing protocols and interference-mitigation techniques that we use in the subnets, are inspired by Ntranos et al. [24]. Transmitters describe quantised versions of transmit signals over the Tx-conferencing links to their leftor right-neighbours, and these neighbours apply dirty-paper coding to mitigate the interference signals described over the conferencing links. Receivers send decoded messages over $\mathrm{Rx}$-conferencing links to their left- or right-neighbours. These neighbours then reconstruct the transmit signals corresponding to the conferenced messages, and subtract these interferences from their receive signals.

In general, the described conferencing strategies are strictly better than conferencing messages at the transmitter-side and quantised versions of receive signals at the receiver-side. The advantage of this latter conferencing strategy however is that it can be applied also in oblivious setups (like for example in C-RANs) where the codebooks are not known during the Tx- and Rx-conferencing phases. When only a single conferencing round is permitted at the transmitter and the receiver side, then the two conferencing strategies are equivalent. In this sense, our results also provide an estimate about the loss in per-user MG in oblivious setups.

\section{APPENDIX A}

\section{DETAILED DESCRIPTION AND ANALYSIS OF THE CODIng SCHEMES IN A SUbNeT}

We describe and analyse the random coding argument that we employ for the first subnet. We will construct random codebooks, which are revealed to all transmitters and receivers before communication starts. The probabilities of decoding errors that we present in our analysis are average probabilities of error, where the average is taken over the random source messages, the random channel realisations and the random choices of the codebooks. We shall identify conditions under which these average probabilities of decoding errors tend to 0 as the blocklength $n \rightarrow \infty$. Standard arguments then imply that there must exist a deterministic choice of all the codebooks such that the average probabilities of decoding errors (now averaged only over the source messages and the channel realisations) tend to 0 as $n \rightarrow \infty$.

Recall that in the first subnet we transmit messages

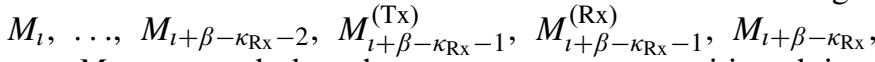
$\ldots, M_{l+\beta-2}$, and that these messages are partitioned into four groups, see subsection V-B.2 and figure 13. We explain transmission of these four groups separately. For ease of exposition, we start with group 1 , followed by group 2, then group 4 , and finally group 3 .

\section{A. Transmission of Messages $M_{l}, \ldots, M_{l+\kappa_{R x}}$}

For each $k \in\left\{l, l+1, \ldots, l+\kappa_{\mathrm{Rx}}\right\}$, let

$$
\mathcal{C}_{\mathrm{P} 2 \mathrm{P}, k}:=\left\{X_{k}^{n}(m)=\left(X_{k, 1}(m) \ldots X_{k, n}(m)\right)\right\}_{m=1}^{\left\lfloor 2^{n R_{k}}\right\rfloor}
$$

be a random Gaussian codebook of rate $R_{k}$ with codewords of length $n$ drawn iid as $X_{k} \sim \mathcal{N}(0, P) .{ }^{8}$ Given source message $M_{k}$, Tx $k$ sends the corresponding codeword $X_{k}^{n}\left(M_{k}\right)$ over the channel.

Consider $\operatorname{Rx} l$ (the first receiver in group one). Tx $(l-1)$ has been deactivated, so the channel output at $\operatorname{Rx} l$ is

$$
Y_{l}^{n}=X_{l}^{n}\left(M_{l}\right)+Z_{l}^{n},
$$

where the addition is understood to be symbol by symbol. Rx $l$ looks through the codebook $\mathcal{C}_{\mathrm{P} 2 \mathrm{P}, l}$ for a unique index $m^{*}$ such that $X_{l}^{n}\left(m^{*}\right)$ and $Y_{l}^{n}$ are jointly typical [38]. If successful, $\mathrm{Rx} l$ declares $\hat{M}_{l}=m^{*}$, otherwise it declares $\hat{M}_{l}=1$. By standard arguments [38], if

$$
R_{l}<\frac{1}{2} \log (1+P),
$$

then $\mathbb{P}\left[\hat{M}_{l} \neq M_{l}\right] \rightarrow 0$ as $n \rightarrow \infty$.

Rx $l$ sends its estimate $\hat{M}_{l}$ of $M_{l}$ to $\mathrm{Rx}(l+1)$ during the first $\mathrm{Rx}$-conferencing round:

$$
V_{l \rightarrow l+1}^{(1)}:=\hat{M}_{l} .
$$

(This will be the only conferencing message that $\operatorname{Rx} l$ sends).

$\operatorname{Rx}(l+1)$ estimates the interference from $\operatorname{Tx} l$ to be $\alpha_{l+1} X_{l}^{n}\left(\hat{M}_{l}\right)$ and computes

$$
\hat{Y}_{l+1}^{n}:=Y_{l+1}^{n}-\alpha_{l+1} X_{l}^{n}\left(\hat{M}_{l}\right) .
$$

If Rx $l$ decoded correctly, $\hat{M}_{l}=M_{l}$, then $X_{l}^{n}\left(\hat{M}_{l}\right)=X_{l}^{n}\left(M_{l}\right)$ and

$$
\hat{Y}_{l+1}^{n}=X_{l+1}^{n}\left(M_{l+1}\right)+Z_{l+1}^{n} .
$$

$\operatorname{Rx}(l+1)$ looks through its codebook $\mathcal{C}_{\mathrm{P} 2 \mathrm{P}, l+1}$ for a unique index $m^{*}$ such that $X_{l+1}^{n}\left(m^{*}\right)$ and $\hat{Y}_{l+1}^{n}$ are jointly typical. If successful, it declares $\hat{M}_{l+1}=m^{*}$, otherwise it declares $\hat{M}_{l+1}=1$. If

$$
R_{l+1}<\frac{1}{2} \log (1+P),
$$

then $\mathbb{P}\left[\hat{M}_{l+1} \neq M_{l+1} \mid \hat{M}_{l}=M_{l}\right] \rightarrow 0$ as $n \rightarrow \infty$.

$\operatorname{Rx}(l+1)$ sends its estimate $\hat{M}_{l+1}$ to $\operatorname{Rx}(l+2)$ during the second Rx-conferencing round:

$$
V_{l+1 \rightarrow l+2}^{(2)}=\hat{M}_{l+1} \text {. }
$$

The same process is repeated for receivers $l+2, \ldots, l+\kappa_{\mathrm{Rx}}$ in increasing order, see also Figure 14. The only difference concerns the Rx-conferencing messages: The right-most receiver $\operatorname{Rx}\left(l+\kappa_{\mathrm{Rx}}-1\right)$ does not send any conferencing message at all. Every other receiver $(l+j)$, for $j \in$ $\left\{2, \ldots, \kappa_{\mathrm{Rx}}-1\right\}$, has to wait until Rx-conferencing round $j+1$ to send its conferencing message $\hat{M}_{l+j}$ :

$$
V_{l+j \rightarrow l+j+1}^{(j+1)}=\hat{M}_{l+j}, \quad j \in\left\{2, \ldots, \kappa_{\mathrm{Rx}}-1\right\} .
$$

The next lemma follows by iteratively applying the arguments we used to derive (75) and (78) and accounting for (76) and (79).

\footnotetext{
${ }^{8}$ To be precise, in order to satisfy the power constraint $P$ in (2), the variance of $X_{k}$ needs to be chosen slightly smaller than $P$. This is a technicality that we will ignore for ease of exposition.
} 
Lemma 2: Source messages $M_{l}, \ldots, M_{l+\kappa_{\mathrm{Rx}}}$ are successfully decoded with probability tending to 1 as $n \rightarrow \infty$, whenever

$$
R_{k}<\frac{1}{2} \log (1+P), \quad \forall k \in\left\{l, \ldots, l+\kappa_{\mathrm{Rx}}\right\},
$$

and

$$
R_{\mathrm{Tx}}>R_{k}, \quad \forall k \in\left\{l, \ldots, l+\kappa_{\mathrm{Rx}}-1\right\}
$$

\section{B. Transmission of Messages $M_{l+\kappa_{R x}+1}, \ldots, M_{l+\kappa_{R x}+\kappa_{T x}}$}

Let us temporarily fix $k=\imath+\kappa_{\mathrm{Rx}}+1$ to simplify notation. Consider Tx $(k-1)$ (the last transmitter in group one), and recall that its channel codeword $X_{k-1}^{n}\left(M_{k-1}\right)$ was chosen from an iid Gaussian codebook of power $P$. To help facilitate communication at $\mathrm{Tx} k$ (the first transmitter of group 2), Tx $(k-1)$ sends a rate $(1 / 2) \log (1+P)$ quantisation of its transmitted signal $X_{k-1}^{n}\left(M_{k-1}\right)$ to Tx $k$.

Let

$$
\hat{X}_{k-1} \sim \mathcal{N}\left(0, \frac{P^{2}}{1+P}\right) \text { and } Z_{k-1}^{\dagger} \sim \mathcal{N}\left(0, \frac{P}{1+P}\right) .
$$

so that

$$
X_{k-1}:=\hat{X}_{k-1}+Z_{k-1}^{\dagger} \sim \mathcal{N}(0, P) .
$$

Construct a random quantisation codebook

$\mathcal{C}_{\mathrm{RD}, k-1}:=\left\{\hat{X}_{k-1}^{n}(u)=\left(\begin{array}{lll}\hat{X}_{k-1,1}(u) & \left.\ldots \hat{X}_{k-1, n}(u)\right)\end{array}\right\}_{u=1}^{\left\lfloor 2^{\left.n R_{\mathrm{Tx}}\right\rfloor}\right.}\right.$

with codewords of length $n$ drawn iid as $\hat{X}_{k-1}$.

Tx $(k-1)$ takes its channel codeword $X_{k-1}^{n}\left(M_{k-1}\right)$ and looks through $\mathcal{C}_{\mathrm{RD}, k-1}$ for a unique index $u^{*}$ such that $X_{k-1}^{n}\left(M_{k-1}\right)$ and $\hat{X}_{k-1}^{n}\left(u^{*}\right)$ are jointly typical. If successful, Tx $(k-1)$ sends the index

$$
U_{k-1 \rightarrow k}^{(1)}=u^{*}
$$

to Tx $k$ during Tx-conferencing round 1 ; otherwise it sends $U_{k-1 \rightarrow k}^{(1)}=1$. (This is the only conferencing message Tx $(k-1)$ sends.)

Let $\mathcal{Q}_{k-1}$ denote the event that the described quantisation is successful, i.e., that there was a unique index $u^{*}$. Standard arguments show that whenever the Tx-conferencing rate satisfies

$$
R_{\mathrm{Tx}}>\frac{1}{2} \log (1+P),
$$

then $\mathbb{P}\left[\mathcal{Q}_{k-1}\right] \rightarrow 1$ as $n \rightarrow \infty$.

Now consider $\mathrm{Tx} / \mathrm{Rx}$ pair $k$. Rx $k$ observes the channel outputs

$$
Y_{k}^{n}=\alpha_{k} X_{k-1}^{n}\left(M_{k-1}\right)+X_{k}^{n}+Z_{k}^{n},
$$

which can be rewritten as

$$
\begin{aligned}
Y_{k}^{n}= & \underbrace{X_{k}^{n}}_{\text {channel input }}+\underbrace{\alpha_{k} \hat{X}_{k-1}^{n}\left(U_{k-1 \rightarrow k}^{(1)}\right)}_{\text {channel state } S_{k}^{n} \text { known at Tx } k} \\
& +\underbrace{\alpha_{k}\left(X_{k-1}^{n}\left(M_{k}\right)-\hat{X}_{k-1}^{n}\left(U_{k-1 \rightarrow k}^{(1)}\right)\right)+Z_{k}^{n}}_{\text {additive noise } \tilde{Z}_{k}^{n}} .
\end{aligned}
$$

After obtaining the conferencing message $U_{k-1 \rightarrow k}^{(1)}$, Tx $k$ reconstructs $\hat{X}_{k-1}\left(U_{k-1 \rightarrow k}^{(1)}\right)$. It then encodes its source message $M_{k}$ using dirty-paper coding over spheres $\left[3\right.$, Sec. V], ${ }^{9}$ [33] of power $P$ and designed for the channel in (83) with additive state sequence

$$
S_{k}^{n}:=\alpha_{k} \hat{X}_{k-1}^{n}\left(U_{k-1 \rightarrow k}^{(1)}\right)
$$

and additive noise sequence

$$
\tilde{Z}_{k}^{n}:=\alpha_{k}\left(X_{k-1}^{n}\left(M_{k}\right)-\hat{X}_{k-1}^{n}\left(U_{k-1 \rightarrow k}^{(1)}\right)\right)+Z_{k}^{n} .
$$

Rx $k$ uses dirty-paper decoding over spheres as described in [3, Sec. V-C]. Adapting [3, Corollary IV.2] similarly as in [3, Remark IV.4], and in view of footnote 10, we obtain the following: If Tx $k$ sends its source message $M_{k}$ to Rx $k$ using dirty-paper coding over spheres $[3, \mathrm{Sec} . \mathrm{V}]^{9},[33]$ of power $P$ and designed for a channel with normalised noise variance $N=1+\alpha_{k}^{2} P /(P+1)$ and interference sequence $S_{k}^{n}$, and if the rate of the source message

$$
R_{k}<\frac{1}{2} \log \left(1+\frac{P}{1+\alpha_{k}^{2} \frac{P}{P+1}}\right),
$$

then $\mathbb{P}\left[\hat{M}_{k} \neq M_{k} \mid \mathcal{Q}_{k-1}\right] \rightarrow 0$ as $n \rightarrow \infty$.

Now let $k=\imath+\kappa_{\mathrm{Rx}}+2$ and consider Tx $(k-1)$ (the first transmitter in group 2 that we also considered in the previous dirty-paper coding step). It facilitates communication at Tx $k$ (the second transmitter in group 2) by sending it a rate$(1 / 2) \log (1+P)$ quantisation of its input signal $X_{k-1}^{n}$. Since this input signal was produced by the dirty-paper coding over spheres in $[3, \mathrm{Sec} . \mathrm{V}])$, we use a quantisation codebook

$$
\mathcal{C}_{\mathrm{RD}, k-1}:=\left\{\hat{X}_{k-1}^{n}(u)\right\}_{u=1}^{\left\lfloor 2^{n R_{\mathrm{Tx}}}\right\rfloor}
$$

with codewords $\hat{X}_{k-1}^{n}(u)$ that are picked iid uniformly over the surface of an $n$-dimensional sphere of radius

$$
\sqrt{n \operatorname{Var}\left(\hat{X}_{k-1}\right)}=\sqrt{n \frac{P^{2}}{1+P}} .
$$

Quantisation is as follows. Tx $(k-1)$ looks through $\mathcal{C}_{\mathrm{RD}, k-1}$ for the vector $\hat{X}_{k-1}^{n}\left(u^{*}\right)$ whos angle with $X_{k-1}^{n}$ is closest to $\sqrt{\frac{P}{P+1}}$ :

$$
u^{*}=\operatorname{argmin}_{u \in\left\{1, \ldots, 2^{n} R_{\mathrm{Tx}}\right\}}\left|\angle\left(\hat{X}_{k-1}^{n}(u), X_{k-1}^{n}\right)-\sqrt{\frac{P}{P+1}}\right| .
$$

Tx $(k-1)$ sends the index $u^{*}$ over the conferencing link to Tx $k$. It does so during the second Tx-conferencing round:

$$
U_{k-1 \rightarrow k}^{(2)}=u^{*} \text {. }
$$

\footnotetext{
${ }^{9}$ Standard dirty-paper coding and its analysis are not sufficient because the noise sequence $Z_{k}^{n}$ is neither iid (not even when averaged over all codebooks) nor independent of the state sequence $S_{k}^{n}$.

In fact, since the state-sequence $S_{k}^{n}$ is not uniform over a sphere, the dirtypaper encoding over spheres needs to be extended as described in the proof of [3, Ramark III-5]. The analysis of this extended dirty-paper coding over spheres only requires that the normalised lengths of the noise and the state sequences are approximately constant and the noise and state sequences are approximately orthogonal [3]. Given event $\mathcal{Q}_{k-1}$, our setup satisfies these conditions: $\frac{1}{n}\left\|\tilde{Z}_{k}^{n}\right\|^{2} \longrightarrow 1+\alpha_{k}^{2} \frac{P}{P+1}, \quad \frac{1}{n}\left\|S_{k}^{n}\right\|^{2} \longrightarrow \alpha_{k}^{2} \frac{P^{2}}{P+1}, \quad$ and $\frac{1}{n}<\tilde{Z}_{k}^{n}, S_{k}^{n}>\longrightarrow 0$, where convergence is in probability everywhere.
} 
By standard arguments (see e.g., proof of [39, eq, (134)]), whenever

$$
R_{\mathrm{Tx}}>\frac{1}{2} \log (1+P)
$$

then

$$
\left|\angle\left(\hat{X}_{k-1}^{n}\left(u^{*}\right), X_{k-1}^{n}\right)-\sqrt{\frac{P}{P+1}}\right| \longrightarrow 0
$$

in probability.

Tx $k$ reconstructs the quantised signal $\hat{X}_{k-1}^{n}\left(U_{k-1 \rightarrow k}^{(2)}\right)$, and encodes its source message $M_{k}$ using dirty-paper coding over spheres of power $P$ and for a channel with normalised noise variance $N=1+\alpha_{k}^{2} P /(P+1)$ and interference sequence $S_{k}^{n}:=\alpha_{k} \hat{X}_{k-1}^{n}\left(U_{k-1 \rightarrow k}^{(2)}\right)$.

Receiver $k$ applies dirty-paper coding over spheres to decode message $M_{k}$ from its output sequence

$$
\begin{aligned}
Y_{k}^{n}= & \underbrace{X_{k}^{n}}_{\text {channel input }}+\underbrace{\alpha_{k} \hat{X}_{k-1}^{n}\left(U_{k-1 \rightarrow k}^{(1)}\right)}_{\text {channel state } S_{k}^{n} \text { known at Tx } k} \\
& +\underbrace{\underbrace{}_{k}\left(X_{k-1}^{n}\left(M_{k}\right)-\hat{X}_{k-1}^{n}\left(U_{k-1 \rightarrow k}^{(1)}\right)\right)+Z_{k}^{n}}_{\text {additive noise } \tilde{Z}_{k}^{n}} .
\end{aligned}
$$

Since given (86) the noise sequence $\tilde{Z}_{k}^{n}$ and state sequence $S_{k}^{n}$ satisfy again the convergence conditions in footnote 10, extending Corollary IV.2 similarly to Remark IV.4 (both in [3]), we obtain that if (86) and

$$
R_{k}<\frac{1}{2} \log \left(1+\frac{P}{1+\alpha_{k}^{2} \frac{P}{P+1}}\right)
$$

hold, then $\mathbb{P}\left[\hat{M}_{k} \neq M_{k}\right] \rightarrow 0$ as $n \rightarrow \infty$.

The same procedure can be repeated for $k=\imath+\kappa_{\mathrm{Rx}}+$ $3, \ldots, l+\kappa_{\mathrm{Rx}}+\kappa_{\mathrm{Tx}}$, see also Figure 15. The next lemma follows by iteratively applying the arguments we used to derive (85) and (88).

Lemma 3: Source messages $M_{l+\kappa_{\mathrm{Rx}}+1} \ldots, M_{l+\kappa_{\mathrm{Rx}}+\kappa_{\mathrm{Tx}}}$ are successfully decoded with probability tending to 1 as $n \rightarrow \infty$, whenever

$$
R_{k}<\frac{1}{2} \log (1+P)
$$

for all $k \in\left\{l+\kappa_{\mathrm{Rx}}+1, \ldots, l+\kappa_{\mathrm{Rx}}+\kappa_{\mathrm{Tx}}\right\}$ and

$$
R_{\mathrm{Tx}}>\frac{1}{2} \log (1+P) \text {. }
$$

C. Transmission of Messages $M_{l+\beta-\kappa_{R x}-1}^{\mathrm{Rx}}, \ldots, M_{l+\beta-2}$

For each $k \in\left\{l+\beta-\kappa_{\mathrm{Rx}}-1, \ldots, l+\beta-2\right\}$, let

$$
\mathcal{C}_{\mathrm{P} 2 \mathrm{P}, k}:=\left\{\Xi_{k}^{n}(m)=\left(\Xi_{k, 1}(m) \ldots \Xi_{k, n}(m)\right)\right\}_{m=1}^{\left\lfloor 2^{\left.n R_{k}\right\rfloor}\right.} .
$$

be a random Gaussian codebook of rate $R_{k}$ with codewords of length $n$ drawn iid as $\Xi_{k} \sim \mathcal{N}\left(0, \alpha_{k+1}^{2} P\right)$. Given source message $M_{k},{ }^{10}$ Tx $k$ picks the corresponding codeword $\Xi_{k}^{n}\left(M_{k}\right)$ and transmits the scaled sequence

$$
X_{k}^{n}=\alpha_{k+1}^{-1} \Xi_{k}^{n}\left(M_{k}\right) .
$$

\footnotetext{
${ }^{10}$ Whenever we write $M_{k}$ or $R_{k}$ for $k=\imath+\beta-\kappa_{\mathrm{Rx}}-1$ in this subsection we actually mean $M_{l+\beta-\kappa_{\mathrm{Rx}}-1}^{\mathrm{Rx}}$ and $R_{l+\beta-\kappa_{\mathrm{Rx}}-1}^{\mathrm{Rx}}$. We do not write the latter for ease of exposition.
}

Transmission of each source message $M_{k}$ goes over the "diagonal path"

$$
\mathrm{Tx} k \quad \longrightarrow \quad \mathrm{Rx}(k+1) \quad \longrightarrow \quad \mathrm{Rx} k .
$$

In fact, $\mathrm{Rx} k+1$ decodes source message $M_{k}$ and describes its guess over the conferencing link to $\operatorname{Rx} k-1$, which then declares this message.

Consider $\operatorname{Rx} l+\beta-1$ (the last receiver in the subnet). Since in our scheme Tx $l+\beta-1$ is deactivated, $\operatorname{Rx} l+\beta-1$ observes

$$
\begin{aligned}
Y_{l+\beta-1}^{n} & =\alpha_{l+\beta-1} X_{l+\beta-2}^{n}+Z_{l+\beta-1}^{n} \\
& =\Xi_{l+\beta-2}^{n}\left(M_{l+\beta-2}\right)+Z_{l+\beta-1}^{n},
\end{aligned}
$$

where the second equality follows by (89).

$\operatorname{Rx} l+\beta-1$ decodes source message $M_{l+\beta-2}$. It looks through codebook $\mathcal{C}_{\mathrm{P} 2 \mathrm{P}, t+\beta-2}$ for a unique index $m^{*}$ such that $\Xi_{l+\beta-2}^{n}\left(m^{*}\right)$ and $Y_{l+\beta-1}^{n}$ are jointly typical. If successful it sets $\hat{M}_{l+\beta-2}=m^{*}$, otherwise it sets $\hat{M}_{l+\beta-2}=1$. By standard arguments [37], if

$$
R_{l+\beta-2}<\frac{1}{2} \log \left(1+\alpha_{l+\beta-1}^{2} P\right),
$$

then $\mathbb{P}\left[\hat{M}_{l+\beta-2} \neq M_{l+\beta-2}\right] \rightarrow 0$ as $n \rightarrow \infty$.

$\operatorname{Rx} \imath+\beta-1$ sends the conferencing message

$$
V_{(l+\beta-1) \rightarrow(l+\beta-2)}^{(1)}=\hat{M}_{l+\beta-2}
$$

to $\operatorname{Rx} l+\beta-2$. (This is the only conferencing message $\operatorname{Rx} l+\beta-1$ sends.)

We next consider $\operatorname{Rx} l+\beta-2$. (This is the receiver immediately left to the previously considered receiver, which obtained the conferencing message (92).) It observes channel outputs

$$
\begin{aligned}
& Y_{l+\beta-2}^{n}=X_{l+\beta-2}^{n}+\alpha_{l+\beta-2} X_{l+\beta-3}^{n}+Z_{l+\beta-2}^{n} \\
& =\underbrace{\alpha_{l+\beta-1}^{-1} \Xi_{l+\beta-2}^{n}\left(M_{l+\beta-2}\right)}_{\text {interference "known" at receiver }}+\underbrace{\Xi_{l+\beta-3}^{n}\left(M_{l+\beta-3}\right)}_{\text {desired signal }} \\
& +\underbrace{Z_{l+\beta-2}^{n}}_{\text {noise }}
\end{aligned}
$$

where the second equality follows again by (89).

$\mathrm{Rx} l+\beta-2$ has a guess of $M_{l+\beta-2}$, see (92), and thus an estimate about $\alpha_{l+\beta-1}^{-1} \Xi_{l+\beta-2}^{n}\left(M_{l+\beta-2}\right)$. It will cancel this "interference" before decoding source message $M_{l+\beta-3}$. Specifically, $\operatorname{Rx} l+\beta-2$ first forms

$$
\hat{Y}_{l+\beta-2}^{n}=Y_{l+\beta-2}^{n}-\alpha_{l+\beta-1}^{-1} \Xi_{l+\beta-2}^{n}\left(\hat{M}_{l+\beta-2}\right),
$$

and then looks through codebook $\mathcal{C}_{\mathrm{P} 2 \mathrm{P}, l+\beta-2}$ for a unique index $m^{*}$ such that $\Xi_{l+\beta-3}^{n}\left(m^{*}\right)$ and $\hat{Y}_{l+\beta-2}^{n}$ are jointly typical. If successful it sets $\hat{M}_{l+\beta-3}=m^{*}$, otherwise it sets $\hat{M}_{l+\beta-3}=1$.

Notice that when $\hat{M}_{l+\beta-2}=M_{l+\beta-2}$, then

$$
\hat{Y}_{l+\beta-2}^{n}=\Xi_{l+\beta-3}^{n}\left(M_{l+\beta-3}\right)+Z_{l+\beta-2}^{n},
$$

and $\operatorname{Rx} l+\beta-2$ can declare source message $M_{l+\beta-3}$ based on an interference-free signal. 
By standard arguments [37], $\mathbb{P}\left[\hat{M}_{l+\beta-3} \quad \neq\right.$ $\left.M_{l+\beta-3} \mid \hat{M}_{l+\beta-2}=M_{l+\beta-2}\right] \rightarrow 0$ as $n \rightarrow \infty$, whenever

$$
R_{l+\beta-3}<\frac{1}{2} \log \left(1+\alpha_{l+\beta-2}^{2} P\right) .
$$

$\operatorname{Rx} l+\beta-2$ sends the Rx-conferencing message

$$
V_{(l+\beta-2) \rightarrow(l+\beta-3)}^{(2)}=\hat{M}_{l+\beta-3}
$$

to its left neighbour. (This is its only conferencing message.)

Finally, Rx $\imath+\beta-2$ declares the guess $\hat{M}_{l+\beta-2}$ that it had obtained from its right neighbour (92).

The same process is repeated for receivers $l+\beta-3$, $\imath+\beta-4, \ldots, i+\beta-\kappa_{\mathrm{Rx}}-1$ in decreasing order.

The next lemma follows by iteratively applying the arguments we used to derive (91) and (96).

Lemma 4: Source messages $M_{l+\beta-\kappa_{\mathrm{Rx}}-1}^{\mathrm{Rx}}, M_{l+\beta-\kappa_{\mathrm{Rx}}}, \ldots$, $M_{l+\beta-2}$ are successfully decoded with probability tending to 1 as $n \rightarrow \infty$, whenever

$$
R_{k}<\frac{1}{2} \log \left(1+\alpha_{k+1}^{2} P\right),
$$

for all $k \in\left\{l+\beta-\kappa_{\mathrm{Rx}}, \ldots, l+\beta-2\right\}$,

$$
R_{l+\beta-\kappa_{\mathrm{Rx}}-1}^{\mathrm{Rx}}<\frac{1}{2} \log \left(1+\alpha_{l+\beta-\kappa_{\mathrm{Rx}}}^{2} P\right),
$$

and

$$
R_{\mathrm{Rx}}>\max \left\{R_{l+\beta-\kappa_{\mathrm{Rx}}-1}^{\mathrm{Rx}}, R_{l+\beta-\kappa_{\mathrm{Rx}}}, \ldots, R_{l+\beta-2}\right\} .
$$

D. Transmission of Messages $M_{l+\beta-\kappa_{\mathrm{Rx}}-\kappa_{T x}}, \ldots$,

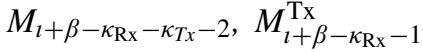

Fix $k=\imath+\beta-\kappa_{\mathrm{Rx}}-1$, and consider the special Tx/Rx pair $k$. (In Figure 13 this is the blue transmitter.) As we described in the previous subsection, Tx $k$ already encoded its source message $M_{k}^{\mathrm{Rx}}$ into its input signal $X_{k}^{n}$ that was drawn from an iid Gaussian codebook.

Tx $k$ now encodes its second source message $M_{k}^{\text {Tx }}$ which it transmits over the path

$$
\mathrm{Tx} k \longrightarrow \mathrm{Tx}(k-1) \longrightarrow \mathrm{Rx} k .
$$

For convenience we will denote $M_{l+\beta-\kappa_{\mathrm{Rx}}-1}^{\mathrm{Tx}}$ simply by $M_{k}$. Rx $k$ will construct a transmit signal $\Phi_{k}^{n}\left(M_{k}\right)$ and send a rate $(1 / 2) \log (1+P)$ quantisation of $\Phi_{k}^{n}$ over the conferencing link to its left-neighbour Tx $(k-1)$. This latter will then reconstruct the quantised sequence $\hat{\Phi}_{k}^{n}$ and send it over the network, see (101) ahead.

We first describe the quantisation, and then the construction of $\Phi_{k}^{n}\left(M_{k}\right)$. Since $\Phi_{k}^{n}\left(M_{k}\right)$ won't be iid Gaussian, we will draw our quantisation codebook uniform over a sphere. Construct a random quantisation codebook

$$
\mathcal{C}_{\mathrm{RD}, k}:=\left\{\hat{\Phi}_{k}^{n}(u)\right\}_{u=1}^{\left\lfloor 2^{n R_{\mathrm{Tx}}}\right\rfloor}
$$

by choosing all vectors iid uniformly over an $n$-dimensional sphere of radius $\sqrt{n \alpha_{k}^{2} P}$. Tx $k$ looks through $\mathcal{C}_{\mathrm{RD}, k}$ for the quantisation vector $\hat{\Phi}_{k}^{n}\left(u^{*}\right)$ whos angle with $\Phi_{k}^{n}$ is closest to $\sqrt{\frac{P}{P+1}}$ :

$$
u^{*}=\operatorname{argmin}\left|\angle\left(\hat{\Phi}_{k}^{n}(u), \Phi_{k}^{n}\right)-\sqrt{\frac{P}{P+1}}\right|,
$$

where the argmin is over all $u \in\left\{1, \ldots, 2^{n R_{\mathrm{Tx}}}\right\}$. Tx $k$ sends index $u^{*}$ to its left-neighbour Tx $k$ in Tx-conferencing round 1 :

$$
U_{k \rightarrow k-1}^{(1)}=u^{*} .
$$

By standard arguments (see e.g., proof of [39, eq. (134)]), whenever

$$
R_{\mathrm{Tx}}>\frac{1}{2} \log (1+P),
$$

then

$$
\left|\angle\left(\hat{\Phi}_{k}^{n}\left(u^{*}\right), \Phi_{k}^{n}\right)-\sqrt{\frac{P}{P+1}}\right| \rightarrow 0
$$

in probability.

After obtaining conferencing message $U_{k \rightarrow k-1}^{(1)}$, Tx $(k-1)$ reconstructs $\hat{\Phi}_{k}\left(U_{k \rightarrow k-1}^{(1)}\right)$ and transmits

$$
X_{k-1}^{n}=\alpha_{k}^{-1} \hat{\Phi}_{k}\left(U_{k \rightarrow k-1}^{(1)}\right)
$$

over the interference network.

Rx $k$ observes the channel outputs

$$
\begin{aligned}
Y_{k}^{n} & =X_{k}^{n}+\alpha_{k} X_{k-1}^{n}+Z_{k}^{n} \\
& =X_{k}^{n}+\hat{\Phi}_{k}^{n}+Z_{k}^{n}
\end{aligned}
$$

which we choose to write

$$
\begin{aligned}
Y_{k}^{n}= & \underbrace{\frac{P}{P+1} \Phi_{k}^{n}\left(M_{k}\right)}_{\text {desired signal }}+\underbrace{X_{k}^{n}}_{\text {state sequence } S_{k}^{n}} \\
& +\underbrace{\left(\hat{\Phi}_{k}^{n}-\frac{P}{P+1} \Phi_{k}^{n}\right)+Z_{k}^{n}}_{\text {noise sequence } \tilde{Z}_{k}^{n}} .
\end{aligned}
$$

Transmitter $k$ encodes $M_{k}$ using a generalized dirty-paper code over spheres [3], [33] of power $\alpha_{k}^{2} \frac{P^{2}}{P+1}$ for the statedependent channel in (106), i.e., for a channel with additive noise

$$
\tilde{Z}_{k}^{n}:=\left(\hat{\Phi}_{k}^{n}-\frac{P}{P+1} \Phi_{k}^{n}\right)+Z_{k}^{n},
$$

and iid additive Gaussian state

$$
S_{k}^{n}:=X_{k}^{n} .
$$

Let $\Psi_{\mathrm{DPC}, k}^{n}\left(M_{k}\right)$ denote the resulting dirty-paper sequence. Tx $k$ sets

$$
\Phi_{k}\left(M_{k}\right):=\frac{P+1}{P} \Psi_{k}^{n}\left(M_{k}\right) .
$$

Receiver $k$ observes

$$
\begin{aligned}
Y_{k}^{n}= & \underbrace{\Psi_{\mathrm{DPC}, k}^{n}\left(M_{k}\right)}_{\text {dirty-paper signal }}+\underbrace{X_{k}^{n}}_{\text {state sequence } S_{k}^{n}} \\
& +\underbrace{\left(\hat{\Phi}_{k}^{n}-\frac{P}{P+1} \Phi_{k}^{n}\right)+Z_{k}^{n}}_{\text {noise sequence } \tilde{Z}_{k}^{n}},
\end{aligned}
$$


and applies dirty-paper decoding over spheres to decode Message $M_{k}$. From a slight extension of [3, Corollary IV.2 $]^{11}$ can be shown to extend readily, e.g., using the arguments in the proof of [3, Remark III-5] we obtain that, if (99) holds and if

$$
R_{k}^{\mathrm{Tx}}<\frac{1}{2} \log \left(1+\frac{\alpha_{k}^{2} \frac{P^{2}}{P+1}}{1+\alpha_{k}^{2} \frac{P}{P+1}}\right),
$$

then $\mathbb{P}\left[\hat{M}_{k} \neq M_{k}\right] \rightarrow 0$ as $n \rightarrow \infty$. The same process is repeated for $k=\imath+\beta-\kappa_{\mathrm{Rx}}-2, \ldots, \imath+\beta-\kappa_{\mathrm{Rx}}-\kappa_{\mathrm{Tx}}$ in decreasing order.

The next lemma follows by iteratively applying the arguments we used to derive (107), see also (99).

$$
\text { Lemma 5: Source messages } M_{l+\beta-\kappa_{\mathrm{Rx}}-\kappa_{\mathrm{TX}}}, \ldots \text {, }
$$
$M_{l+\beta-\kappa_{\mathrm{Rx}}-2}, \quad M_{l+\beta-\kappa_{\mathrm{Rx}}-1}^{\mathrm{Tx}}$ are successfully decoded with probability tending to 1 as $n \rightarrow \infty$, whenever

$$
R_{k}<\frac{1}{2} \log \left(1+\frac{\alpha_{k}^{2} \frac{P^{2}}{P+1}}{1+\alpha_{k}^{2} \frac{P}{P+1}}\right)
$$

for $k \in\left\{l+\beta-\kappa_{\mathrm{Rx}}-\kappa_{\mathrm{Tx}}, \ldots, l+\beta-\kappa_{\mathrm{Rx}}-2\right\}$ and

$$
R_{l+\beta-\kappa_{\mathrm{Rx}}-1}^{\mathrm{Tx}}<\frac{1}{2} \log \left(1+\frac{\frac{P^{2}}{P+1} \alpha_{l+\beta-\kappa_{\mathrm{Rx}}-1}^{2}}{1+\frac{P}{P+1} \alpha_{l+\beta-\kappa_{\mathrm{Rx}}-1}^{2}}\right) .
$$

and

$$
R_{\mathrm{Tx}}>\frac{1}{2} \log (1+P)
$$

\section{REFERENCES}

[1] A. N. Barreto et al., "5G-wireless communications for 2020," J. Commun. Inf. Syst., vol. 31, no. 1, pp. 146-163, 2016.

[2] F. M. J. Willems, "The discrete memoryless multiple access channel with partially cooperating encoders," IEEE Trans. Inf. Theory, vol. 29, no. 3, pp. 441-445, May 1983.

[3] S. I. Bross, A. Lapidoth, and M. Wigger, "Dirty-paper coding for the Gaussian multiaccess channel with conferencing," IEEE Trans. Inf. Theory, vol. 58, no. 9, pp. 5640-5668, Sep. 2012.

[4] S. I. Bross, A. Lapidoth, and M. A. Wigger, "The Gaussian MAC with conferencing encoders," in Proc. Int. Symp. Inf. Theory (ISIT), Toronto, ON, Canada, Jul. 2008, pp. 2702-2706.

[5] M. A. Wigger and G. Kramer, "Three-user MIMO MACs with cooperation," in Proc. Inf. Theory Workshop (ITW), Volos, Greece, Jun. 2009, pp. 221-225.

[6] I. Maric, R. D. Yates, and G. Kramer, "Capacity of interference channels with partial transmitter cooperation," IEEE Trans. Inf. Theory, vol. 53, no. 10, pp. 3536-3548, Oct. 2007.

[7] O. Simeone, O. Somekh, G. Kramer, H. V. Poor, and S. Shamai (Shitz), "Three-user Gaussian multiple access channel with partially cooperating encoders," in Proc. Asilomar Conf. Signals, Syst. Comput., Oct. 2008, pp. $85-89$.

[8] R. Dabora and S. D. Servetto, "Broadcast channels with cooperating decoders," IEEE Trans. Inf. Theory, vol. 52, no. 12, pp. 5438-5454, Dec. 2006.

[9] R. Dabora and S. D. Servetto, "A multi-step conference for cooperative broadcast," in Proc. Int. Symp. Inf. Theory (ISIT), Seattle, WA, USA, Jul. 2006, pp. 2190-2194.

${ }^{11}$ The extension is required because the state sequence $S_{k}^{n}$ is not uniform over a sphere and because the noise is not independent of the input. We however have the following limits (in probability): $\frac{1}{n}\left\|\Phi_{k}^{n}\left(M_{k}\right)\right\| \longrightarrow \alpha_{k}^{2}(P+$ 1) and $\frac{1}{n}\left\|S_{k}^{n}\right\|^{2} \longrightarrow P$. Moreover when (99) holds, then by (100) also $\frac{1}{n}\left\|\tilde{Z}_{k}^{n}\right\|^{2} \longrightarrow 1+\alpha_{k}^{2} \frac{P}{P+1}, \frac{1}{n}<\tilde{Z}_{k}^{n}, S_{k}^{n}>\longrightarrow 0$, and $\frac{1}{n}<\tilde{Z}_{k}^{n}, \Psi_{\mathrm{DPC}, k}^{n}>$ $\stackrel{n}{\longrightarrow} 0$ (all in probability). Under these assumptions, [3, Corollary IV.2
[10] C. T. K. Ng, I. Maric, A.J. Goldsmith, R. D. Yates, and S. Shamai (Shitz), "Iterative and one-shot conferencing in relay channels," in Proc. Inf. Theory Workshop, Punta del Este, Uruguay, Mar. 2006, pp. 193-197.

[11] P. Noorzad, M. Effros, and M. Langberg. (Jan. 2016). "The unbounded benefit of encoder cooperation for the $k$-user MAC." [Online]. Available: http://arxiv.org/abs/1601.06113

[12] Y. Steinberg, "Instances of the relay-broadcast channel and cooperation strategies," in Proc. Int. Symp. Inf. Theory (ISIT), Hong Kong, Jun. 2015, pp. 2653-2657.

[13] H. Mao, W. Feng, and N. Ge, "Receiver cooperation for MIMO broadcast channels with finite-rate feedback," IEEE Commun. Lett., vol. 19, no. 5, pp. 887-890, May 2015.

[14] S. Salehkalaibar and M. R. Aref, "On the capacity region of a class of Z Channels with cooperation," in Proc. Int. Symp. Inf. Theory Appl. (ISITA), Taichung, China, Oct. 2010, pp. 464-468.

[15] Z. Goldfeld, H. H. Permuter, and G. Kramer, "Duality of a source coding problem and the semi-deterministic broadcast channel with rate-limited cooperation," IEEE Trans. Inf. Theory, vol. 62, no. 5, pp. 2285-2307, May 2016.

[16] I.-H. Wang and D. N. C. Tse, "Interference mitigation through limited transmitter cooperation," IEEE Trans. Inf. Theory, vol. 57, no. 5, pp. 2941-2965, May 2011.

[17] I.-H. Wang and D. N. C. Tse, "Interference mitigation through limited receiver cooperation," IEEE Trans. Inf. Theory, vol. 57, no. 5, pp. 2913-2940, May 2011.

[18] A. D. Wyner, "Shannon-theoretic approach to a Gaussian cellular multiple-access channel," IEEE Trans. Inf. Theory, vol. 40, no. 6, pp. 1713-1727, Nov. 1994

[19] S. V. Hanly and P. A. Whiting, "Information-theoretic capacity of multireceiver networks," Telecommun. Syst., vol. 1, pp. 1-42, Dec. 1993.

[20] O. Simeone et al., "Cooperative wireless cellular systems: An information-theoretic view," Found. Trends Commun. Inf. Theory, vol. 8, nos. 1-2, pp. 1-177, 2012.

[21] J. Xu, J. Zhang, and J. G. Andrews, "When does the Wyner model accurately describe an uplink cellular network?" in Proc. IEEE GLOBECOM Wireless Commun. Symp., Miami, FL, USA, Dec. 2010, pp. 1-5.

[22] N. Levy and S. Shamai (Shitz), "Clustered local decoding for Wynertype cellular models," IEEE Trans. Inf. Theory, vol. 55, no. 11, pp. 4976-4985, Nov. 2009.

[23] A. Lapidoth, S. Shamai (Shitz), and M. A. Wigger, "On cognitive interference networks," in Proc. Inf. Theory Workshop (ITW), Lake Tahoe, United States of America, Sep. 2007, pp. $325-330$.

[24] V. Ntranos, M. A. Maddah-Ali, and G. Caire, "Cellular interference alignment," IEEE Trans. Inf. Theory, vol. 61, no. 3, pp. 1194-1217, Mar. 2015.

[25] V. Ntranos, M. A. Maddah-Ali, and G. Caire, "Cellular interference alignment: Omni-directional antennas and asymmetric configurations," IEEE Trans. Inf. Theory, vol. 61, no. 12, pp. 6663-6679, Dec. 2015.

[26] V. Ntranos, M. A. Maddah-Ali, and G. Caire. (Jul. 2014). "On uplink-downlink duality for cellular IA." [Online]. Available: http://arxiv.org/abs/1407.3538

[27] B. He, N. Yang, X. Zhou, and J. Yuan, "Base station cooperation for confidential broadcasting in multi-cell networks," IEEE Trans. Wireless Commun., vol. 14, no. 10, pp. 5287-5299, Oct. 2015.

[28] V. S. Annapureddy, A. El Gamal, and V. V. Veeravalli, "Degrees of freedom of interference channels with CoMP transmission and reception," IEEE Trans. Inf. Theory, vol. 58, no. 9, pp. 5740-5760, Sep. 2015.

[29] S. Shamai (Shitz) and M. Wigger, "Rate-limited transmittercooperation in Wyner's asymmetric interference network," in Proc. Int. Symp. Inf. Theory (ISIT), St. Petersburg, Russia, Jul./Aug. 2011, pp. $425-429$.

[30] A. Lapidoth, N. Levy, S. Shamai (Shitz), and M. Wigger, "Cognitive Wyner networks with clustered decoding," IEEE Trans. Inf. Theory, vol. 60, no. 10, pp. 6342-6367, Oct. 2014.

[31] V. R. Cadambe and S. A. Jafar, "Interference alignment and degrees of freedom of the $K$-user interference channel," IEEE Trans. Inf. Theory, vol. 54, no. 8, pp. 3425-3441, Aug. 2008.

[32] A. El Gamal, "Cell associations that maximize the average uplinkdownlink degrees of freedom," in Proc. Int. Symp. Inf. Theory, Barcelona, Spain, Jun. 2016, pp. 1461-1465.

[33] A. S. Cohen and A. Lapidoth, "The Gaussian watermarking game," IEEE Trans. Inf. Theory, vol. 48, no. 6, pp. 1639-1667, Jun. 2002. 
[34] M. Costa, "Writing on dirty paper (Corresp.)," IEEE Trans. Inf. Theory, vol. 29, no. 3, pp. 439-441, May 1983.

[35] D. Slepian and J. K. Wolf, "A coding theorem for multiple access channels with correlated sources," Bell Syst. Tech. J., vol. 52, no. 7, pp. 1037-1076, 1973.

[36] M. Bande, A. El Gamal, and V. V. Veeravalli, "Flexible backhaul design with cooperative transmission in cellular interference networks," in Proc. Int. Symp. Inf. Theory, Hong Kong, Jun. 2015, pp. 2431-2435.

[37] T. M. Cover and J. A. Thomas, Elements of Information Theory, 2nd ed. New York, NY, USA: Wiley, 2006.

[38] A. El Gamal and Y.-H. Kim, Network Information Theory. Cambridge, U.K.: Cambridge Univ. Press, 2011.

[39] A. Lapidoth and S. Tinguely, "Sending a bivariate Gaussian over a Gaussian MAC," IEEE Trans. Inf. Theory, vol. 56, no. 6, pp. 2714-2752, Jun. 2010.

[40] H. Sato, "The capacity of the Gaussian interference channel under strong interference (Corresp.)," IEEE Trans. Inf. Theory, vol. 27, no. 6, pp. 786-788, Nov. 1981.

[41] G. Kramer, "Outer bounds on the capacity of Gaussian interference channels," IEEE Trans. Inf. Theory, vol. 50, no. 3, pp. 581-586, Mar. 2004.

Michèle Wigger (S'05-M'09-SM'14) received the M.Sc. degree in electrical engineering, with distinction, and the $\mathrm{Ph} . \mathrm{D}$. degree in electrical engineering both from ETH Zurich in 2003 and 2008, respectively. In 2009, she was first a post-doctoral fellow at the University of California, San Diego, USA, and then joined Telecom Paris Tech, Paris, France, where she is currently an Associate Professor. Dr. Wigger has held visiting professor appointments at the Technion-Israel Institute of Technology and ETH Zurich.

Dr. Wigger has previously served as an Associate Editor of the IEEE COMMUNICATION LETTERS, and is now Associate Editor for Shannon Theory of the IEEE TRANSACTIONS ON INFORMATION THEORY. She is currently also serving on the Board of Governors of the IEEE Information Theory Society. Dr. Wigger's research interests are in multi-terminal information theory, in particular in distributed source coding and in capacities of networks with states, feedback, user cooperation, or caching.
Roy Timo (S'06-M'09) is an Experienced Researcher at Ericsson Research in Stockholm, Sweden. Prior to joining Ericsson, he was an Alexander von Humboldt Research Fellow with the Institute for Communications Engineering at the Technische Universität München (2014-2016); a Research Fellow with the Institute for Telecommunications Research at the University of South Australia (2008-2013); and a Postdoctoral Researcher with the Department of Communications and Electronics at Telecom ParisTech (2013-2014). He received the Bachelor of Engineering and Ph.D. degrees from The Australian National University in 2005 and 2009, respectively.

Shlomo Shamai (Shitz) (S'80-M'82-SM'88-F'94) During 1975-1985 he was with the Communications Research Labs, in the capacity of a Senio Research Engineer. Since 1986 he is with the Department of Electrical Engineering, Technion-Israel Institute of Technology, where he is now a Technion Distinguished Professor, and holds the William Fondiller Chair of Telecommunications. His research interests encompasses a wide spectrum of topics in information theory and statistical communications.

Dr. Shamai (Shitz) is an IEEE Fellow, a member of the Israeli Academy of Sciences and Humanities and a foreign member of the US National Academy of Engineering. He is the recipient of the 2011 Claude E. Shannon Award, the 2014 Rothschild Prize in Mathematics/Computer Sciences and Engineering and the 2017 IEEE Richard W. Hamming Medal.

He has been awarded the 1999 van der Pol Gold Medal of the Union Radio Scientifique Internationale (URSI), and is a co-recipient of the 2000 IEEE Donald G. Fink Prize Paper Award, the 2003, and the 2004 joint IT/COM societies paper award, the 2007 IEEE Information Theory Society Paper Award, the 2009 and 2015 European Commission FP7, Network of Excellence in Wireless COMmunications (NEWCOM++, NEWCOM\#) Best Paper Awards, the 2010 Thomson Reuters Award for International Excellence in Scientific Research, the 2014 EURASIP Best Paper Award (for the EURASIP Journal on Wireless Communications and Networking), and the 2015 IEEE Communications Society Best Tutorial Paper Award. He is also the recipient of 1985 Alon Grant for distinguished young scientists and the 2000 Technion Henry Taub Prize for Excellence in Research. He has served as Associate Editor for the Shannon Theory of the IEEE TRANSACTIONS ON INFORMATION THEORY, and has also served twice on the Board of Governors of the Information Theory Society. He has also served on the Executive Editorial Board of the IEEE TRANSACTIONS ON INFORMATION THEORY and on the IEEE Information Theory Society Nominations and Appointments Committee. 